\title{
TENSOR STRUCTURES ARISING FROM AFFINE LIE ALGEBRAS. I
}

\author{
D. KAZHDAN AND G. LUSZTIG
}

\section{INTRODUCTION}

Let $\mathbf{g}$ be a simple Lie algebra of type $A, D$, or $E$. There is a natural central extension of Lie algebras $0 \rightarrow \mathbf{C 1} \rightarrow \widehat{\mathbf{g}} \rightarrow \mathbf{C}((\epsilon)) \otimes \mathbf{g} \rightarrow 0$ (see 1.3) which has been extensively studied by physicists and mathematicians. ( 1 is a distinguished element of the centre.) A $\widehat{\mathbf{g}}$-module is said to have central charge $\kappa^{\prime} \in \mathbf{C}$ if $\mathbf{1}$ acts on it as multiplication by $\kappa^{\prime}$. For any $\kappa \in \mathbf{C}^{*}$, we denote by $\widetilde{\mathscr{O}}_{\kappa}$ the category of $\widehat{\mathbf{g}}$-modules of finite length, with central charge $\kappa-h$ (where $h$ is the Coxeter number) which are integrable in the sense of $\mathrm{Kac}[\mathrm{K}]$. It is known that $\widetilde{\mathscr{O}}_{\kappa}$ is semisimple; moreover, it is nonzero only if $\kappa$ is an integer $\geq h$. In the work of physicists (Belavin, Polyakov, and Zamolodchikov [BPZ], Knizhnik and Zamolodchikov [KZ], Moore and Seiberg [MS]) it has been realized that the category $\widetilde{\mathscr{O}}_{\kappa}$ has an additional structure, namely, that of a rigid braided tensor category. This work of physicists has been put on a rigurous mathematical foundation by Tsuchiya and Kanie [TK], Tsuchiya, Ueno, and Yamada [TUY], and independently by Beilinson and Feigin (unpublished; but see [BFM] for a discussion of the closely related Virasoro case). However, the precise construction of the tensor structure on $\widetilde{\mathscr{\sigma}}_{\kappa}$ has not been given in the literature.

In this paper we are interested in a category $\mathscr{O}_{\kappa}$ of $\widehat{\mathbf{g}}$-modules which is larger than $\widetilde{\mathscr{O}}_{\kappa}$ : it consists of modules with central charge $\kappa-h$, of finite length, whose composition factors are simple highest weight modules corresponding to weights which are dominant in the direction of $\mathbf{g}$. (The simple modules in this category are in 1-1 correspondence with the simple finite-dimensional g-modules.)

We show that, in the case where $\kappa \notin \mathbf{Q}_{>0}$, this category has a natural structure of braided tensor category; the rigidity will be shown in a sequel to this paper. (There is no such structure on $\mathscr{O}_{\kappa}$ in the case where $\kappa \in \mathbf{Q}_{>0}$.)

We will show in another paper how this tensor structure may be used to establish an equivalence of categories between $\mathscr{O}_{\kappa}$ and the category of finitedimensional integrable representations of a quantum group with parameter $e^{\sqrt{-1} \pi / \kappa}$. (See the announcement in [KL].)

Received by the editors October 5, 1992.

1991 Mathematics Subject Classification. Primary 20G99.

Both authors are supported in part by the National Science Foundation. 
The most interesting case is that where $\kappa \in \mathbf{Q}_{<0}$; in this case, the category $\mathscr{O}_{\kappa}$ is not semisimple, just like that of finite-dimensional representations of a quantum group at a root of 1 .

One important source of inspiration for our work was the work of Drinfeld [D] in which he showed that the tensor category of the finite-dimensional representations of the quantized enveloping algebra corresponding to $\mathbf{g}$, with formal parameter, is equivalent to a tensor category whose objects are the finitedimensional representations of $\mathbf{g}$ and whose tensor structure is obtained from the Knizhnik-Zamolodchikov equations. (From our point of view, this last category is essentially $\mathscr{O}_{\kappa}$ for $\kappa$ in a formal neighbourhood of $\infty$.)

We now describe the content of this paper in more detail. The category $\mathscr{O}_{\kappa}$ is studied in $\S \S 2$ and 3; various equivalent definitions for it are given there, and the duality functor is introduced. In $\S \S 4-6$ we give three constructions of the tensor product; the first two are equivalent, but the third one is the same as the first two only under suitable finiteness assumptions (as shown in $\S 7$ ). In $\S 8$ we study the effect of changing the ground ring in a tensor product.

\section{CONTENTS}

1. Preliminaries

2. The category $\mathscr{O}_{\kappa}$

3. A characterization of $\mathscr{O}_{\kappa}$

4. First definition of tensor product

5. Second definition of tensor product

6. Third definition of tensor product

7. Finiteness for tensor products

8. Change of rings in tensor products

\section{Preliminaries}

1.1. Modules over a Lie algebra. Let $A$ be a commutative $\mathbf{C}$-algebra with 1 . Let $\mathbf{h}$ be a Lie algebra over $A$ or an $A$-Lie algebra; we denote by $U(\mathbf{h})$ the enveloping algebra (over $A$ ) of $\mathbf{g}$.

An h-module is, by definition, an $A$-module $V$ together with a homomorphism $\mathbf{h} \rightarrow \operatorname{End}_{A}(V)$ of $A$-Lie algebras; this is the same as a $U(\mathbf{h})$-module.

If $A \rightarrow B$ is a homomorphism of $\mathbf{C}$-algebras with 1 , we may regard $B \otimes_{A} \mathbf{h}$ naturally as a $B$-Lie algebra. If $V$ is a h-module, then $B \otimes_{A} V$ is naturally a $B \otimes_{A}$ h-module.

1.2. The Lie algebra $\mathbf{g}$. Let $\mathbf{g}$ be a semisimple Lie algebra over $\mathbf{C}$ with the following property: $\mathbf{g}$ is isomorphic to a direct sum of copies of $a$ single simple Lie algebra of type $A, D$, or $E$.

Let $h$ be the Coxeter number of any of the simple components of $\mathbf{g}$.

Let $():, \mathbf{g} \times \mathbf{g} \rightarrow \mathbf{C}$ be the $\mathbf{g}$-invariant bilinear form defined as $(2 h)^{-1}$ times the Killing form.

We shall assume, as we may, that $\mathbf{g}$ is given in terms of the generators $e_{i}, f_{i}, h_{i} \quad(i \in I)$ and the Serre relations $\left[e_{i}, f_{j}\right]=\delta_{i j} h_{i} ;\left[h_{i}, e_{j}\right]=a_{i j} e_{j}$, $\left[h_{i}, f_{j}\right]=-a_{i j} f_{j} ;\left[h_{i}, h_{j}\right]=0 ;\left[e_{i},\left[e_{i}, e_{j}\right]\right]=\left[f_{i},\left[f_{i}, f_{j}\right]\right]=0$ if $a_{i j}=-1$; and $\left[e_{i}, e_{j}\right]=\left[f_{i}, f_{j}\right]=0$ if $a_{i j}=0$. Here $\left(a_{i j}\right)_{i, j \in I}$ is the Cartan matrix. 
The form $($,$) satisfies \left(h_{i}, h_{j}\right)=a_{i j}$ and $\left(e_{i}, f_{j}\right)=\delta_{i j}$.

Let $\mathbf{g}^{-}$be the Lie subalgebra of $\mathbf{g}$ generated by the elements $f_{i} \quad(i \in I)$.

Let $\mathbf{Z}^{I}$ (resp. $\mathbf{N}^{I}$ ) be the set of all maps $I \rightarrow \mathbf{Z}($ resp. $I \rightarrow \mathbf{N})$. Let $\left(b_{i j}\right)$ be the matrix inverse to $\left(a_{i j}\right)$; for $a, b \in \mathbf{Z}^{I}$, we set $\langle a, b\rangle=\sum_{i, j} b_{i j} a(i) b(j)$.

For any $a \in \mathbf{N}^{I}$, let $\mathscr{V}_{a}=U\left(\mathbf{g}^{-}\right) /\left(\sum_{i} U\left(\mathbf{g}^{-}\right) f_{i}^{a(i)+1}\right)$. Let $y_{a}$ be the image of $1 \in U\left(\mathbf{g}^{-}\right)$in $\mathscr{V}_{a}$. There is a unique g-module structure on $\mathscr{V}_{a}$ such that $\mathbf{g}^{-}$acts by left multiplication and $e_{i} y_{a}=0$ and $h_{i}\left(y_{a}\right)=a(i) y_{a}$ for all $i \in I$. This g-module is simple and finite dimensional.

The g-module dual to $\mathscr{V}_{a}$ is isomorphic to $\mathscr{V}_{\bar{a}}$ where $a \rightarrow \bar{a}$ is an involution of $\mathbf{N}^{I}$. There is a well-defined involution $i \mapsto \bar{i}$ of $I$ such that $\bar{a}(i)=a(\bar{i})$ for all $i \in I$.

1.3. The Lie algebras $\widehat{\mathbf{g}}_{A}, \widetilde{\mathbf{g}}_{A}$. Let $A$ be a commutative $\mathbf{C}$-algebra with 1 . Let $\epsilon$ be an indeterminate. Let $A 1$ be the free $A$-module of rank 1 with basis element 1 .

For $f_{1}, f_{2} \in A((\epsilon))$ we define $\left\{f_{1}, f_{2}\right\} \in A$ to be the residue at $\epsilon=0$ of the formal differential form $f_{2} d\left(f_{1}\right)$. We have $\left\{f_{1}, f_{2}\right\}+\left\{f_{2}, f_{1}\right\}=0$ and $\left\{f_{1} f_{2}, f_{3}\right\}+\left\{f_{2} f_{3}, f_{1}\right\}+\left\{f_{3} f_{1}, f_{2}\right\}=0$ for all $f_{1}, f_{2}, f_{3} \in A((\epsilon))$. Hence

$$
\widehat{\mathbf{g}}_{A}=A((\epsilon)) \otimes \mathbf{g} \oplus A \mathbf{1}
$$

is an $A$-Lie algebra with bracket

$$
\left[f c, f^{\prime} c^{\prime}\right]=f f^{\prime}\left[c, c^{\prime}\right]+\left\{f, f^{\prime}\right\}\left(c, c^{\prime}\right) \mathbf{1}, \quad[f c, \mathbf{1}]=0, \quad[\mathbf{1}, \mathbf{1}]=0
$$

for $f, f^{\prime} \in A((\epsilon))$ and $c, c^{\prime} \in \mathbf{g}$. (We shall often write $f c$ instead of $f \otimes c$.)

Let $\widetilde{\mathbf{g}}_{A}=A\left[\epsilon, \epsilon^{-1}\right] \otimes \mathbf{g} \oplus A \mathbf{1}$. This is naturally an $A$-sub-Lie algebra of $\widehat{\mathbf{g}}_{A}$. Its bracket can also be described by the formula

$$
\left[\epsilon^{n} c, \epsilon^{m} c^{\prime}\right]=\epsilon^{n+m}\left[c, c^{\prime}\right]+\delta_{n+m, 0} n\left(c, c^{\prime}\right) \mathbf{1}
$$

for any $n, m \in \mathbf{Z}$ and any $c, c^{\prime} \in \mathbf{g}$, together with the requirement that $\mathbf{1}$ is in the centre.

$\widetilde{\mathbf{g}}_{A}$ is called the affine Lie algebra over $A$.

Let $\widehat{\mathbf{g}}_{A}^{+}$be the $A$-Lie subalgebra $A[[\epsilon]] \otimes \mathbf{g} \oplus A \mathbf{1}$ of $\widehat{\mathbf{g}}_{A}$. Let $\widetilde{\mathbf{g}}_{A}^{+}$be the $A$-Lie subalgebra $A[\epsilon] \otimes \mathbf{g} \oplus A \mathbf{1}$ of $\widetilde{\mathbf{g}}_{A}$.

In the case where $A=\mathrm{C}$ we shall often omit $A$ in our notation; for example, we write $\widehat{\mathbf{g}}, \widetilde{\mathbf{g}}$ instead of $\widehat{\mathbf{g}}_{A}, \widetilde{\mathbf{g}}_{A}$ and $\otimes$ instead of $\otimes_{A}$.

If $A \rightarrow B$ is a homomorphism of $A$ into another commutative $\mathbf{C}$-algebra with 1 , then the obvious homomorphism $B \otimes_{A} \widetilde{\mathbf{g}}_{A} \rightarrow \widetilde{\mathbf{g}}_{B}$ is an isomorphism of $B$-Lie algebras. On the other hand, the obvious homomorphism $B \otimes_{A} \widehat{\mathbf{g}}_{A} \rightarrow \widehat{\mathbf{g}}_{B}$ is not necessarily an isomorphism; one case when it is an isomorphism, is when $A=\mathrm{C}$ and $B$ is finite dimensional as a $\mathbf{C}$-vector space.

1.4. If $\mathbf{g}^{\prime}$ is a Lie subalgebra of $\mathbf{g}$ which is a sum of some of the simple components of $\mathbf{g}$, then we have an obvious imbedding of $A$-Lie algebras $\widehat{\mathbf{g}}_{A}^{\prime} \rightarrow$ $\widehat{\mathbf{g}}_{A}$ given by $f c \mapsto f c, \mathbf{1} \mapsto \mathbf{1}$ for all $f \in A((\epsilon))$ and $c \in \mathbf{g}^{\prime}$. This restricts to an imbedding of $A$-Lie algebras $\widetilde{\mathbf{g}}_{A}^{\prime} \rightarrow \widetilde{\mathbf{g}}_{A}$. 
1.5. Let ${ }^{\sharp}: \widetilde{\mathbf{g}}_{A} \rightarrow \widetilde{\mathbf{g}}_{A}$ be the $A$-Lie algebra involution defined by $\left(\epsilon^{n} c\right)^{\sharp}=$ $(-\epsilon)^{-n} c$ for all $n \in \mathbf{Z}, c \in \mathbf{g}$ and by $(\mathbf{1})^{\sharp}=-\mathbf{1}$.

Let $V$ be the $\widetilde{\mathbf{g}}_{A}$-module defined by the Lie algebra homomorphism $\widetilde{\mathbf{g}}_{A} \rightarrow$ End $_{A}(V)$. Composing this with the involution $\sharp: \widetilde{\mathbf{g}}_{A} \rightarrow \widetilde{\mathbf{g}}_{A}$, we obtain a new Lie algebra homomorphism $\widetilde{\mathbf{g}}_{A} \rightarrow$ End $_{A}(V)$, and this defines a new $\widetilde{\mathbf{g}}_{A}$-module structure on $V$, denoted $V^{\sharp}$.

1.6. If $S$ is a finite set, then $\mathbf{g}^{S}=\mathbf{g} \oplus \cdots \oplus \mathbf{g}$ (summands in 1-1 correspondence with the elements of $S$ ) is naturally a semisimple Lie algebra of the kind considered in 1.2. Hence all definitions and results given in this paper for $\mathbf{g}$ are automatically applicable to $\mathbf{g}^{S}$.

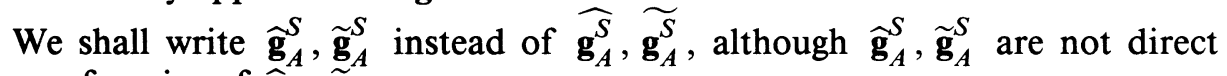
sums of copies of $\widehat{\mathbf{g}}_{A}, \widetilde{\mathbf{g}}_{A}$.

For each $s \in S$, the imbedding $\mathbf{g} \subset \mathbf{g}^{S}$ of the $s$-component gives rise, as in 1.4 , to $A$-Lie algebra imbeddings $\delta_{s}: \widehat{\mathbf{g}}_{A} \rightarrow \widehat{\mathbf{g}}_{A}^{S}$ and $\delta_{s}: \widetilde{\mathbf{g}}_{A} \rightarrow \widetilde{\mathbf{g}}_{A}^{S}$.

Taking the sum over $s \in S$ we get surjective $A$-Lie algebra homomorphisms $\left(\widehat{\mathbf{g}}_{A}\right)^{S} \rightarrow \widehat{\mathbf{g}}_{A}^{S}$ and $\left(\widetilde{\mathbf{g}}_{A}\right)^{S} \rightarrow \widetilde{\mathbf{g}}_{A}^{S}$ with kernels consisting of the elements $\left(d_{s} \mathbf{1}\right)_{s \in S}$ with $\left(d_{s}\right) \in A^{S}$ such that $\sum_{s} d_{s}=0$. $\left(\xi_{s}\right)$.

We shall sometimes write the element $\sum_{s} \delta_{s}\left(\xi_{s}\right)$ of $\widehat{\mathbf{g}}_{A}^{S}$ (where $\xi_{s} \in \widehat{\mathbf{g}}_{A}$ ) as

We have a natural isomorphism $A((\epsilon))^{S} \otimes \mathbf{g} \oplus A \mathbf{1} \cong \widehat{\mathbf{g}}_{A}^{S}$; it takes $\mathbf{1}$ to $\mathbf{1}$ and associates to $g \otimes c$ the element $\left(g_{s} c\right)$, where $g=\left(g_{s}\right) \in A((\epsilon))^{S}$ and $c \in \mathbf{g}$.

We shall sometimes write $g c$ instead of $\left(g_{s} c\right)$.

1.7. For any integer $N \geq 1$, we define $Q_{N}$ to be the $A$-submodule of $U\left(\widetilde{\mathbf{g}}_{A}\right)$ generated by the products $\left(\epsilon c_{1}\right)\left(\epsilon c_{2}\right) \cdots\left(\epsilon c_{N}\right)$ with $c_{1}, c_{2}, \ldots, c_{N}$ in $\mathbf{g}$. We also define $Q_{0}$ to be the $A$-submodule of $U\left(\widetilde{\mathbf{g}}_{A}\right)$ generated by the unit element 1 . Let $Q_{N}^{\sharp}$ be the image of $Q_{N}$ under the involution ${ }^{\sharp}$.

Lemma 1.8. (a) $\epsilon^{p} c \in Q_{p}$ if $p \geq 1$ and $c \in \mathbf{g}$.

(b) $Q_{n}\left(\epsilon^{-p} c\right) \in \sum_{j=0}^{p-1}\left(\epsilon^{-p+j} \mathbf{g}\right) Q_{n-j}+\mathbf{g} Q_{n-p}+Q_{n-p}+1 Q_{n-p}$ if $n \geq p \geq 0$ and $c \in \mathbf{g}$.

(c) Let $f \in A[[\epsilon]]$ and $c \in \mathbf{g}$. We have $(f c) Q_{t}^{\sharp} \subset \sum_{t^{\prime}=0}^{t} Q_{t^{\prime}}^{\sharp} U\left(\widehat{\mathbf{g}}_{A}^{+}\right)$.

The proofs of (a), (b) are left to the reader. We prove (c). We argue by induction on $t \geq 0$. The case where $t=0$ is trivial; hence, we may assume that $t \geq 1$.

Let $x \in Q_{t}^{\sharp}$, and assume that $x=\left(\epsilon^{-1} c^{\prime}\right) x^{\prime}$ where $x^{\prime} \in Q_{t-1}^{\sharp}$ and $c^{\prime} \in \mathbf{g}$. We have

$$
\begin{aligned}
(f c) x & =(f c)\left(\epsilon^{-1} c^{\prime}\right) x^{\prime} \\
& =\left(\epsilon^{-1} c^{\prime}\right)(f c) x^{\prime}+\left(f \epsilon^{-1}\left[c, c^{\prime}\right]\right) x^{\prime}+\left(c, c^{\prime}\right) \operatorname{Res}_{0}\left(\epsilon^{-1} d(f)\right) \mathbf{1} x^{\prime}
\end{aligned}
$$

Consider the three terms in the last sum. By the induction hypothesis, the first term is in $Q_{1}^{\sharp} \sum_{t^{\prime}=0}^{t-1} Q_{t^{\prime}}^{\sharp} U\left(\widehat{\mathbf{g}}_{A}^{+}\right) \subset \sum_{t^{\prime}=0}^{t} Q_{t^{\prime}}^{\sharp} U\left(\widehat{\mathbf{g}}_{A}^{+}\right)$. 
The second term is of the form $f(0)\left(\epsilon^{-1}\left[c, c^{\prime}\right]\right) x^{\prime}+\left(\tilde{f}\left[c, c^{\prime}\right]\right) x^{\prime}$ (where $f=$ $f(0)+\epsilon \tilde{f})$ and hence is in $Q_{t}^{\sharp}+\sum_{t^{\prime}=0}^{t-1} Q_{t^{\prime}}^{\sharp} U\left(\widehat{\mathbf{g}}_{A}^{+}\right)$(by the induction hypothesis).

The third term is in $Q_{t-1}^{\sharp} U\left(\widehat{\mathbf{g}}_{A}^{+}\right)$. It follows that $(f c) x \in \sum_{t^{\prime}=0}^{t} Q_{t^{\prime}}^{\sharp} U\left(\widehat{\mathbf{g}}_{A}^{+}\right)$, as required.

1.9. Given a $\widetilde{\mathbf{g}}_{A}$-module $V$ and an integer $N$ we define an $A$-submodule $V(N)$ of $V$ as follows. If $N \geq 0$, we set $V(N)=\left\{x \in V \mid Q_{N} x=0\right\}$. If $N \leq 0$, we set $V(N)=\left\{x \in V \mid Q_{-N}^{\sharp} x=0\right\}$. For $N=0$, the two definitions coincide: we have $V(0)=0$. We have $V(0) \subset V(1) \subset V(2) \subset \cdots$; we set $V(\infty)=\bigcup_{N \geq 0} V(N)$. We have $V(0) \subset V(-1) \subset V(-2) \subset \cdots$; we set $V(-\infty)=\bigcup_{N \leq 0} V(N)$. Clearly,

$$
V(\infty)=V^{\sharp}(-\infty) \text {. }
$$

For any $N \geq 1$ we denote by $V[N]$ the set of all $x \in V$ such that $\left(\epsilon^{n} c\right) x=0$ for all $n \geq N$ and all $c \in \mathbf{g}$. We have $V[1] \subset V[2] \subset \cdots$; we set $V[\infty]=$ $\bigcup_{N} V[N]$.

The elements of $V(\infty)$ are called the smooth elements of $V$. We say that the $\widetilde{\mathbf{g}}$-module $V$ is smooth if all its elements are smooth, i.e., if $V=V(\infty)$.

Lemma 1.10. (a) $V(\infty)$ is a $\widetilde{\mathbf{g}}_{A}$-submodule of $V$; if $N \geq 0$, then $V(N)$ is a $\widetilde{\mathbf{g}}_{A}^{+}$-submodule of $V$.

(b) $V(-\infty)$ is a $\widetilde{\mathbf{g}}_{A}$-submodule of $V$.

(c) $V(N) \subset V[N]$ for any $N \geq 1$; hence, $V(\infty) \subset V[\infty]$.

(d) If $N \geq 2$, then we have a natural exact sequence

$$
0 \longrightarrow V(1) \longrightarrow V(N) \longrightarrow \operatorname{Hom}_{\mathbf{C}}(\mathbf{g}, V(N-1))
$$

the last arrow associates to $x \in V(N)$ the linear map $c \mapsto(\epsilon c) x, \forall c \in \mathbf{g}$.

(e) If $A$ is Noetherian and $V(1)$ is a finitely generated A-module, then $V(N)$ is a finitely generated $A$-module for any $N \geq 1$.

Assume that $N \geq 0$. For any $p \geq 0$, we have $Q_{N}\left(\epsilon^{p} c\right) \subset U\left(\widetilde{\mathbf{g}}_{A}\right) Q_{N}$ (see 1.8(a), (b)); hence, $V(N)$ is stable under $\left(\epsilon^{p} c\right): V \rightarrow V$. We also have $Q_{N} \mathbf{1}=1 Q_{N}$; hence, $V(N)$ is stable under $1: V \rightarrow V$. Thus, $V(N)$ is a $\widetilde{\mathbf{g}}_{A}^{+}$-submodule. It also follows that $V(\infty)$ is a $\widetilde{\mathbf{g}}_{A}^{+}$-submodule. Note that $Q_{N}\left(\epsilon^{-p} c\right) \subset \sum_{j=0}^{p} U\left(\widetilde{\mathbf{g}}_{A}\right) Q_{N-j}($ see $1.8(\mathrm{~b}))$ if $N \geq p$. This implies that $V(\infty)$ is stable under $\left(\epsilon^{-p} c\right): V \rightarrow V$ and (a) is proved. Now (b) follows from (a) using the involution $\sharp$ of $\widetilde{\mathbf{g}}_{A}$.

If $N \geq 1$ and $x \in V(N)$ then $\left(\epsilon^{p} c\right) x=0$ for all $c \in \mathbf{g}$ and all $p \geq N$ by 1.8(a). This proves (c). Now (d) is obvious; (e) follows from (d) by induction on $N$. The lemma is proved.

1.11. Any smooth $\widetilde{\mathbf{g}}_{A}$-module $V$ (or, more generally, a $\widetilde{\mathbf{g}}_{A}$-module $V$ such that $V=V[\infty])$ can be naturally extended to a $\widehat{\mathbf{g}}_{A}$-module as follows. Let $x \in V[N]$ and let $f \in A((\epsilon)), c \in \mathbf{g}$; we define $(f c) x=\left(f_{1} c\right) x$ where $f_{1} \in A\left[\epsilon, \epsilon^{-1}\right]$ is such that $f-f_{1} \in \epsilon^{N} A[[\epsilon]]$. The operators $(f c): V \rightarrow V$ are independent of choices and, together with the operator $\mathbf{1}: V \rightarrow V$ of the $\widetilde{\mathbf{g}}_{A}$-module structure, define a $\widehat{\mathbf{g}}_{A}$-module structure on $V$. 
1.12. Let $\kappa^{\prime} \in \mathbf{C}$. A $\widetilde{\mathbf{g}}_{A}$-module $V$ is said to have central charge $\kappa^{\prime}$ if $\mathbf{1} \in \widetilde{\mathbf{g}}_{A}$ acts on $V$ as multiplication by $\kappa^{\prime}$.

1.13. Let $V_{s} \quad(s \in S)$ be $\widetilde{\mathbf{g}}_{A}$-modules which have the same central charge $\kappa^{\prime} \in$ C. Then $\bigotimes_{s \in S} V_{s}$ (tensor product over $A$ ) has a unique $\widetilde{\mathbf{g}}_{A^{S}}^{S}$-module structure such that for any $\xi=\left(\xi_{s}\right) \in \widetilde{\mathbf{g}}_{A}^{S}$ and any family of elements $x_{s^{\prime}} \in V_{s^{\prime}} \quad\left(s^{\prime} \in S\right)$ we have $\xi\left(\bigotimes_{s^{\prime} \in S} x_{s^{\prime}}\right)=\sum_{s \in S}\left(\bigotimes_{s^{\prime} \in S} x_{s, s^{\prime}}\right)$ where $x_{s, s^{\prime}} \in V_{s^{\prime}}$ is equal to $\xi_{s}\left(x_{s}\right)$ if $s^{\prime}=s$ and to $x_{s^{\prime}}$ if $s^{\prime} \neq s$.

To see this, we must only check that, if $\xi_{s}=d_{s} \mathbf{1} \in \widetilde{\mathbf{g}}_{A}$ with $d_{s} \in A$ satisfying $\sum_{s} d_{s}=0$, then the definition above gives zero. But in this case, we have $x_{s, s^{\prime}}=\kappa^{\prime} d_{s} x_{s^{\prime}}$ if $s^{\prime}=s$ and $\sum_{s \in S}\left(\bigotimes_{s^{\prime} \in S} x_{s, s^{\prime}}\right)=\kappa^{\prime} \sum_{s} d_{s}\left(\bigotimes_{s^{\prime} \in S} x_{s^{\prime}}\right)=0$, as required.

Note that the $\widetilde{\mathbf{g}}_{A}^{S}$-module $\bigotimes_{S} V_{S}$ has central charge $\kappa^{\prime}$. It is clear that, if each $V_{s}$ is smooth, then the $\widetilde{\mathbf{g}}_{A}^{S}$-module $\bigotimes_{S} V_{s}$ is smooth. In that case, the $\widetilde{\mathbf{g}}_{A}^{S}$-module $\otimes_{S} V_{S}$ can be regarded naturally as a $\widehat{\mathbf{g}}_{A}^{S}$-module, as in 1.11 .

1.14. Let $V$ be a $\widetilde{\mathbf{g}}_{A}$-module such that $V=V[\infty]$ and such that $V$ has central charge $\kappa-h$ where $\kappa \in \mathbf{C}$ is nonzero. ( $h$ is as in 1.2.) For any $k \in \mathbf{Z}$, the Sugawara operator $L_{k}: V \rightarrow V$ is given by

(a) $\quad L_{k}(x)=\frac{1}{2 \kappa} \sum_{j \geq-k / 2} \sum_{p}\left(\epsilon^{-j} c_{p}\right)\left(\epsilon^{j+k} c_{p}\right) x+\frac{1}{2 \kappa} \sum_{j<-k / 2} \sum_{p}\left(\epsilon^{j+k} c_{p}\right)\left(\epsilon^{-j} c_{p}\right) x$

where $\left(c_{p}\right)$ is a basis of $\mathbf{g}$ such that $\left(c_{p}, c_{p^{\prime}}\right)=\delta_{p p^{\prime}}$. This operator is well defined (only finitely many terms are nonzero for any fixed $x$ ) and independent of the choice of basis. We have

$$
\left(\epsilon^{n} c\right)\left(L_{k} x\right)-L_{k}\left(\left(\epsilon^{n} c\right) x\right)=n\left(\epsilon^{n+k} c\right) x
$$

for any $x \in V$, any $c \in \mathbf{g}$, and any $n \in \mathbf{Z}$ and

$$
\left[L_{0}, L_{-1}\right]=L_{-1}, \quad\left[L_{0}, L_{1}\right]=-L_{1},
$$$$
\left[L_{1}, L_{-1}\right]=2 L_{0}
$$

(See $[\mathrm{KR}]$.)

1.15. If $\mathbf{g}$ is replaced by $\mathbf{g}^{\star}$ for some finite set $\boldsymbol{k}$, and if $V$ is as above (for $\mathbf{g}^{\boldsymbol{\alpha}}$ ), then we can restrict the $\widetilde{\mathbf{g}}_{A}^{\boldsymbol{\alpha}}$-module $V$ to a $\widetilde{\mathbf{g}}_{A}$-module, via $\delta_{t}: \widetilde{\mathbf{g}}_{A} \rightarrow \widetilde{\mathbf{g}}_{A}^{\boldsymbol{\alpha}}$, where $t \in \boldsymbol{A}$. The Sugawara operator $L_{k}$ for this restriction is denoted $L_{k ; t}$. Clearly, the Sugawara operator of the $\widetilde{\mathbf{g}}_{A}^{\boldsymbol{\alpha}}$-module $V$ is given by

$$
L_{k}=\sum_{t \in \mathfrak{d}} L_{k ; t} .
$$

1.16. For any $A$-module $V$ we set ${ }^{d} V=\operatorname{Hom}_{A}(V, A)$; this is again an $A$ module. If $V$ is, moreover, a $\widetilde{\mathbf{g}}_{A}$-module, then we regard ${ }^{d} V$ with the $\widetilde{\mathbf{g}}_{A}$ module structure induced from that of $V$; thus, $(\xi(\mu))(x)=-\mu(\xi(x))$ for all $\xi \in \widetilde{\mathbf{g}}_{A}, \mu \in{ }^{d} V$, and $x \in V$.

For any $\widetilde{\mathbf{g}}_{A}$-module $V$ we define a $\widetilde{\mathbf{g}}_{A}$-module $D(V)={ }^{d} V^{\sharp}(\infty)$. Note that $\left({ }^{d} V\right)^{\sharp}={ }^{d}\left(V^{\sharp}\right)$ as $\widetilde{\mathbf{g}}_{A}$-modules; this justifies omitting the brackets. 
Note that $V \mapsto D(V)$ is naturally a contravariant functor from $\widetilde{\mathbf{g}}_{A}$-modules to $\widetilde{\mathbf{g}}_{A}$-modules.

Assume that $V$ is a smooth $\widetilde{\mathbf{g}}_{A}$-module. We have an obvious $A$-linear map $V \rightarrow{ }^{d}(D(V))$ : to any $x \in V$ we associate the linear form $f \mapsto f(x)$ on $D(V)$. We may regard the same as a map $V \rightarrow{ }^{d}(D(V))^{\sharp}$; this is now a $\widetilde{\mathbf{g}}_{A}$-module homomorphism. Hence it takes $V(\infty)$ into ${ }^{d}(D(V))^{\sharp}$ and hence defines a $\widetilde{\mathbf{g}}_{A^{-}}$ module homomorphism

$$
V \rightarrow D(D(V))
$$

since $V=V(\infty)$.

Let $x \in V$ and let $\mu \in D(V)$. We have, using the definitions,

$$
\mu\left(L_{0}(x)\right)=\left(L_{0}(\mu)\right)(x) .
$$

\section{THE CATEGORY $\mathscr{O}_{\kappa}$}

In this section we will introduce a category $\mathscr{O}_{K}$ of $\widetilde{\mathbf{g}}$-modules with central charge $\kappa-h$, where $\kappa \notin \mathbf{Q}_{\geq 0}$. (See 2.15.) We will give several alternative descriptions for it (see 2.22) and show that it is stable under the duality $D$.

2.1. We consider a module $\mathscr{N}$ over the $\mathbf{C}$-Lie algebra $\mathbf{C}[\epsilon] \otimes \mathbf{g}$. We say that $\mathscr{N}$ is a nil-module if

(a) $\operatorname{dim}_{\mathbf{C}} \mathscr{N}<\infty$, and

(b) there exists $t \geq 1$ such that the product in the enveloping algebra of any $t$ elements of $\epsilon \mathbf{C}[\epsilon] \otimes \mathbf{g}$ acts on $\mathscr{N}$ as zero.

Using Engel's theorem, we see that the condition (b) is equivalent to the conjunction of the following two conditions:

(b1) there exists $t \geq 1$ such that each element of $\epsilon^{t} \mathbf{C}[\epsilon] \otimes \mathbf{g}$ acts on $\mathscr{N}$ as zero, and

(b2) any element of $\epsilon \mathbf{C}[\epsilon] \otimes \mathbf{g}$ acts on $\mathscr{N}$ as a nilpotent transformation.

2.2. From Engel's theorem we see also that for a nil-module $\mathscr{N}$, there exists a finite filtration $\mathscr{N}=\mathscr{N}_{0} \supset \mathscr{N}_{1} \supset \cdots$ by submodules such that on each successive quotient the Lie algebra $\epsilon \mathbf{C}[\epsilon] \otimes \mathbf{g}$ acts as zero.

2.3. We shall fix $\kappa \in \mathbf{C}^{*}$. Given a nil-module $\mathscr{N}$ as above, we extend $\mathscr{N}$ to a $\widetilde{\mathbf{g}}^{+}$-module by defining the action of $\mathbf{1} \in \widetilde{\mathbf{g}}^{+}$to be multiplication by $\kappa-h$. (Recall (1.2) that $h$ is the Coxeter number.) Let

$$
\mathscr{N}^{\kappa}=U(\widetilde{\mathbf{g}}) \otimes_{U\left(\widetilde{\mathbf{g}}^{+}\right)} \mathscr{N}
$$

be the induced $\widetilde{\mathbf{g}}$-module. We say that $\mathscr{N}^{\kappa}$ is a generalized Weyl module. Note that we have a canonical imbedding $\mathscr{N} \subset \mathscr{N}^{\kappa}$ as a $\widetilde{\mathbf{g}}^{+}$-module and its image generates $\mathscr{N}^{\kappa}$ as a $\widetilde{\mathbf{g}}$-module.

Clearly, the $\widetilde{\mathbf{g}}$-module $\mathscr{N}^{\kappa}$ has central charge $\kappa-h$.

Note that, for any $\widetilde{\mathbf{g}}$-module $W$ with central charge $\kappa-h$, composition with the canonical imbedding defines an isomorphism

$$
\operatorname{Hom}_{\widetilde{\mathbf{g}}}\left(\mathscr{N}^{\kappa}, W\right) \cong \operatorname{Hom}_{\mathbf{g}[\epsilon]}(\mathcal{N}, W) .
$$


2.4. We apply the previous definitions in the case where $\mathscr{N}=\mathscr{V}_{a}$ (see 1.2) for some $a \in \mathbf{N}^{I}$, regarded as a $\mathbf{C}[\epsilon] \otimes \mathbf{g}$-module with $\epsilon \mathbf{C}[\epsilon] \otimes \mathbf{g}$ acting as zero. In this case, the $\widetilde{\mathbf{g}}$-module $\mathscr{N}^{\kappa}$ is denoted by $\mathbf{V}_{a}^{\kappa}$; it is called a Weyl module. By definition,

$$
\mathbf{V}_{a}^{\kappa}=U(\widetilde{\mathbf{g}}) \otimes_{U\left(\widetilde{\mathbf{g}}^{+}\right)} \mathscr{V}_{a}
$$

where $\mathscr{V}_{a}$ is regarded as a $\tilde{\mathbf{g}}^{+}$-module with $\epsilon \mathbf{C}[\epsilon] \otimes \mathbf{g}$ acting as zero and $\mathbf{1}$ acting as multiplication by $\kappa-h$.

Lemma 2.5. (a) For any generalized Weyl module $\mathscr{N}^{\kappa}$ there exists a finite filtration by submodules such that the successive quotients are Weyl modules.

(b) Let $V$ be a $\widetilde{\mathbf{g}}$-module with central charge $\kappa-h$. Assume that there exists $N \geq 1$ such that $\operatorname{dim} V(N)<\infty$ and $V(N)$ generates $V$ as a $\widetilde{\mathbf{g}}$-module. Then $V$ is a quotient of a generalized Weyl module $\mathscr{N}_{\kappa}$.

(a) follows immediately from 2.2 , using the exactness of induction (which in turn follows from the Poincaré-Birkhoff-Witt theorem).

We now prove (b).

By $1.10(\mathrm{a}), V(N)$ is a $\mathbf{C}[\epsilon] \otimes$ g-submodule of $V$; it is contained in $V[N]$ (see $1.10(\mathrm{c}))$, and, hence, $\left(\epsilon^{N} \mathbf{C}[\epsilon]\right) \otimes \mathbf{g}$ acts on $V(N)$ as zero. We show that a product in $U(\widetilde{\mathbf{g}})$ of any $N$ elements of $(\epsilon \mathbf{C}[\epsilon]) \otimes \mathbf{g}$ acts on $V(N)$ as zero. It suffices to show that any product of form $\left(\epsilon^{t_{1}} c_{1}\right)\left(\epsilon^{t_{2}} c_{2}\right) \cdots\left(\epsilon^{t_{N}} c_{N}\right)$ with $t_{1} \geq$ $1, t_{2} \geq 1, \ldots$ acts on $V(N)$ as zero. But, by $1.8(\mathrm{a})$, this product is contained in $Q_{t_{1}} Q_{t_{2}} \cdots Q_{t_{N}} \subset Q_{t_{1}+t_{2}+\cdots+t_{N}}$ and so it acts as zero on $V(N)$ since $t_{1}+t_{2}+\cdots \geq$ $N$.

Thus, the $\mathbf{C}[\epsilon] \otimes \mathbf{g}$-module $\mathscr{N}=V(N)$ is a nil-module. Then the generalized Weyl module $\mathscr{N}^{\kappa}$ is well defined and it has a canonical $\widetilde{\mathbf{g}}$-homomorphism into $V$ which induces the identity map on $\mathscr{N}=V(N)$. Its image contains $V(N)$; hence, it is the whole $V$ since $V(N)$ generates $V$ as a $\widetilde{\mathbf{g}}$-module. This proves (b).

2.6. The following result concerns the action of the Sugawara operator $L_{0}$ on a Weyl module $\mathbf{V}_{a}^{\kappa}$. For any $\lambda \in \mathbf{C}$, we set ${ }_{\lambda} \mathbf{V}_{a}^{\kappa}=\left\{y \in \mathbf{V}_{a}^{\kappa} \mid L_{0}(y)=\lambda y\right\}$.

Proposition 2.7. (a) We have $\mathbf{V}_{a}^{\kappa}=\bigoplus_{\lambda \in \mathbf{C}}\left({ }_{\lambda} \mathbf{V}_{a}^{\kappa}\right)$. In other words, the endomorphism $L_{0}: \mathbf{V}_{a}^{\kappa} \rightarrow \mathbf{V}_{a}^{\kappa}$ is locally finite and semisimple.

(b) $\operatorname{dim}_{\lambda} \mathbf{V}_{a}^{\kappa}<\infty$ for any $\lambda \in \mathbf{C}$.

(c) If $\lambda_{\lambda} \mathbf{V}_{a}^{\kappa} \neq 0$, then $\lambda=\frac{1}{2 \kappa}\langle a, a+2\rangle+n$ for some $n \in \mathbf{N}$.

(d) If $\lambda=\frac{1}{2 \kappa}\langle a, a+2\rangle$, then ${ }_{\lambda} \mathbf{V}_{a}^{\kappa}$ is equal to $\mathscr{V}_{a}$ and, hence, is nonzero.

(e) Any proper $\widetilde{\mathbf{g}}$-submodule $V^{\prime}$ of $\mathbf{V}_{a}^{\kappa}$ is contained in the subspace $\bigoplus_{\lambda}\left({ }_{\lambda} \mathbf{V}_{a}^{\kappa}\right)$ where $\lambda$ runs over the set $\left\{\frac{1}{2 \kappa}\langle a, a+2\rangle+n \mid n=1,2, \ldots\right\}$.

(f) We have $\mathbf{V}_{a}^{\kappa}=\mathbf{V}_{a}^{\kappa}(\infty)$.

Let $\left(c_{p}\right)$ be a basis of $\mathbf{g}$ such that $\left(c_{p}, c_{p^{\prime}}\right)=\delta_{p p^{\prime}}$. Let $y$ be a vector in the subspace $\mathscr{T}_{a}$ of $\mathbf{V}_{a}^{\kappa}$. We have $\left(\epsilon^{j} c_{p}\right) y=0$ for all $p$ and all $j>0$ and, hence,

$$
L_{0}(y)=\frac{1}{2 \kappa} \sum_{p} c_{p} c_{p}(y)=\frac{1}{2 \kappa}\langle a, a+2\rangle y ;
$$


the last equality is a well-known property of the Casimir operator on $\mathscr{V}_{a}$.

The vector space $\mathbf{V}_{a}^{\kappa}$ is spanned by the vectors

$$
\left(\epsilon^{-n_{1}} c_{1}^{\prime}\right) \cdots\left(\epsilon^{-n_{r}} c_{r}^{\prime}\right)(y)
$$

for various $c_{1}^{\prime}, c_{2}^{\prime}, \ldots, c_{r}^{\prime} \in \mathbf{g}$ and $n_{1}, n_{2}, \ldots, n_{r} \in \mathbf{N}$. Such a vector is contained in ${ }_{\lambda} \mathbf{V}_{a}^{\kappa}$ where $\lambda=\frac{1}{2 \kappa}\langle a, a+2\rangle+n_{1}+\cdots+n_{r}$ (by (g) and 1.14(b)). Hence (a)-(c) hold. We also see that ${ }_{\lambda} \mathbf{V}_{a}^{\kappa}$ (for $\lambda=\frac{1}{2 \kappa}\langle a, a+2\rangle$ ) is spanned by the vectors above with $n_{1}=\cdots=n_{r}=0$; hence, it is equal to $\mathscr{V}_{a}$. This proves (d).

In the setup of (e), $V^{\prime}$ is clearly stable under the endomorphism $L_{0}: \mathbf{V}_{a}^{\kappa} \rightarrow$ $\mathbf{V}_{a}^{\kappa}$; hence, by (a), it is the sum of its intersections with the various ${ }_{\lambda} \mathbf{V}_{a}^{\kappa}$. It is enough to show that, for $\lambda=\frac{1}{2 \kappa}\langle a, a+2\rangle$, the intersection $V^{\prime} \cap{ }_{\lambda} \mathbf{V}_{a}^{\kappa}=V^{\prime} \cap \mathscr{V}_{a}$ is zero. This intersection is clearly a g-submodule of $\mathscr{V}_{a}$. If it is nonzero it must be equal to $\mathscr{V}_{a}$ (which is simple); thus, $\mathscr{V}_{a} \subset V^{\prime}$. But $\mathscr{V}_{a}$ generates $\mathbf{V}_{a}^{\kappa}$ as a $\widetilde{\mathbf{g}}$-module; it follows that $V^{\prime}=\mathbf{V}_{a}^{\kappa}$, a contradiction. This proves (e).

We now prove (f). Clearly, $\mathscr{V}_{a} \subset \mathbf{V}_{a}^{\kappa}(1)$; hence, $\mathscr{V}_{a} \subset \mathbf{V}_{a}^{\kappa}(\infty)$. Since $\mathbf{V}_{a}^{\kappa}(\infty)$ is a $\widetilde{\mathbf{g}}$-submodule of $\mathbf{V}_{a}^{\kappa}$ and $\mathscr{V}_{a}$ generates the $\widetilde{\mathbf{g}}$-module $\mathbf{V}_{a}^{\kappa}$, it follows that $\mathbf{V}_{a}^{\kappa}=\mathbf{V}_{a}^{\kappa}(\infty)$. The proposition is proved.

2.8. Part (e) of the previous proposition shows that for $\kappa \neq 0$ the $\widetilde{\mathbf{g}}$-module $\mathbf{V}_{a}^{\kappa}$ has a unique maximal submodule. The quotient of $\mathbf{V}_{a}^{\kappa}$ by this maximal submodule is denoted $\mathbf{L}_{a}^{\kappa}$. This is a simple $\widetilde{\mathbf{g}}$-module. The natural surjective map $\mathbf{V}_{a}^{\kappa} \rightarrow \mathbf{L}_{a}^{\kappa}$ restricts to an injective map $\mathscr{V}_{a} \rightarrow \mathbf{L}_{a}^{\kappa}$ (by 2.7(e)); we use it to identify $\mathscr{V}_{a}$ with a subspace of $\mathbf{L}_{a}^{\kappa}$.

Lemma 2.9. For any $a \in \mathbf{N}^{I}$, we have

$$
\mathbf{L}_{a}^{\kappa}(1)=\mathscr{V}_{a}
$$

In particular, if $a, a^{\prime} \in \mathbf{N}^{I}$, the $\tilde{\mathbf{g}}$-modules $\mathbf{L}_{a}^{\kappa}, \mathbf{L}_{a^{\prime}}^{\kappa}$ are isomorphic if and only if $a=a^{\prime}$.

The inclusion $\mathscr{V}_{a} \subset \mathbf{L}_{a}^{\kappa}(1)$ is obvious. Assume that this inclusion is strict. Using the definitions, we see that $\mathbf{L}_{a}^{\kappa}(1)$ is $\mathbf{g}$-stable. The action of $\mathbf{g}$ on $\mathbf{L}_{a}^{\kappa}(1)$ is locally finite (it is locally finite on $\mathbf{V}_{a}^{\kappa}$ by $2.7(\mathrm{a})$, (b); hence, it is locally finite on the quotient $\mathbf{L}_{a}^{\kappa}$ of $\mathbf{V}_{a}^{\kappa}$, and hence it is locally finite on the subspace $\mathbf{L}_{a}^{\kappa}(1)$ of $\mathbf{L}_{a}^{\kappa}$ ). By the complete reducibility of finite-dimensional g-modules, we can find a finite-dimensional simple g-submodule $\mathscr{V}^{\prime}$ of $\mathbf{L}_{a}^{\kappa}(1)$, whose intersection with $\mathscr{V}_{a}$ is zero. Then, for some $b \in \mathbf{N}^{I}$, there is an isomorphism of g-modules $\mathscr{V}_{b} \cong \mathscr{V}^{\prime}$. Since $\mathscr{V}^{\prime} \subset \mathbf{L}_{a}^{\kappa}(1)$, there is a unique homomorphism of $\widetilde{\mathbf{g}}$-modules $\mathbf{V}_{b}^{\kappa} \rightarrow \mathbf{L}_{a}^{\kappa}$ whose restriction to $\mathscr{V}_{b}$ is the isomorphism $\mathscr{V}_{b} \cong \mathscr{V}^{\prime}$ just considered (see 2.3(a)). This homomorphism has image which is a simple $\widetilde{\mathbf{g}}$-module; hence, it factors through a homomorphism of $\widetilde{\mathbf{g}}$-modules $\mathbf{L}_{b}^{\kappa} \rightarrow \mathbf{L}_{a}^{\kappa}$ (necessarily an isomorphism) which carries $\mathscr{V}_{b}$ onto $\mathscr{V}^{\prime}$. By $2.7(\mathrm{c})$, we have that $\mathscr{V}_{a}$ is the $\lambda$-eigenspace of $L_{0}: \mathbf{L}_{a}^{\kappa} \rightarrow \mathbf{L}_{a}^{\kappa}$, where $\lambda=\frac{1}{2 \kappa}\langle a, a+2\rangle$. By the same result for 
$b$ instead of $a$, we have that $\mathscr{V}_{b}$ is the $\lambda^{\prime}$-eigenspace of $L_{0}: \mathbf{L}_{b}^{\kappa} \rightarrow \mathbf{L}_{b}^{\kappa}$, where $\lambda^{\prime}=\frac{1}{2 \kappa}\langle b, b+2\rangle$. The isomorphism $\mathbf{L}_{b}^{\kappa} \cong \mathbf{L}_{a}^{\kappa}$ is compatible with the actions of $L_{0}$. Hence $\lambda^{\prime}$ is an eigenvalue of $L_{0}: \mathbf{L}_{a}^{\kappa} \rightarrow \mathbf{L}_{a}^{\kappa}$ (necessarily different from $\lambda$, since $\left.\mathscr{V}^{\prime} \cap \mathscr{V}_{a}=0\right)$, and, similarly, $\lambda$ is an eigenvalue of $L_{0}: \mathbf{L}_{b}^{\kappa} \rightarrow \mathbf{L}_{b}^{\kappa}$ (necessarily different from $\lambda^{\prime}$ ). By $2.7(\mathrm{~d})$, we then have $\lambda^{\prime}-\lambda \in\{1,2, \ldots\}$; by the same result for $b$ instead of $a$, we have $\lambda-\lambda^{\prime} \in\{1,2, \ldots\}$. This is a contradiction; (a) is proved. The lemma follows.

Lemma 2.10. Let $W, W^{\prime}$ be $\widetilde{\mathbf{g}}$-submodules of $\mathbf{V}_{a}^{\kappa}$ such that $W \subset W^{\prime}$ and $W \neq W^{\prime}$. There exists $a^{\prime} \in \mathbf{N}^{I}$ such that:

(a) the $\frac{1}{2 \kappa}\left\langle a^{\prime}, a^{\prime}+2\right\rangle$-eigenspace of $L_{0}: W^{\prime} / W \rightarrow W^{\prime} / W$ is nonzero, and

(b) $\frac{1}{2 \kappa}\left(\left\langle a^{\prime}, a^{\prime}+2\right\rangle-\langle a, a+2\rangle\right) \in \mathbf{N}$.

$W, W^{\prime}$ are stable under $L_{0}: \mathbf{V}_{a}^{\kappa} \rightarrow \mathbf{V}_{a}^{\kappa}$ and $L_{0}$ induces semisimple operators on $W, W^{\prime}$, and $W^{\prime} / W$. (See 2.7(a).) By 2.7(c), we can find an eigenvalue $\lambda \in \mathbf{C}$ of $L_{0}: W^{\prime} / W \rightarrow W^{\prime} / W$ with minimum possible real part. If $x$ is a vector in the corresponding eigenspace, then for any $c \in \mathbf{g}$ we have that $L_{0}((\epsilon c) x)=(\epsilon c)\left(L_{0} x\right)-(\epsilon c) x=(\lambda-1)(\epsilon c) x$. By the choice of $\lambda$ we then have $(\epsilon c) x=0$. Thus we have $x \in\left(W^{\prime} / W\right)(1)$. Thus the intersection of the $\lambda$ eigenspace of $L_{0}: W^{\prime} / W \rightarrow W^{\prime} / W$ with $\left(W^{\prime} / W\right)(1)$ is nonzero. This intersection is a finite-dimensional g-submodule. It follows that there exists $a^{\prime} \in \mathbf{N}^{I}$ and a homomorphism of $\widetilde{\mathbf{g}}$-modules $\mathbf{V}_{a^{\prime}}^{\kappa} \rightarrow W^{\prime} / W$ which maps the subspace $\mathscr{V}_{a^{\prime}}$ injectively into that intersection. Using now $2.7(\mathrm{~d})$, it follows that $\lambda=\frac{1}{2 \kappa}\left\langle a^{\prime}, a^{\prime}+2\right\rangle$.

Applying 2.7(c) to $\mathbf{V}_{a}^{\kappa}$, we see that $\lambda=\frac{1}{2 \kappa}\langle a, a+2\rangle+n$ for some $n \in \mathbf{N}$. The lemma is proved.

Lemma 2.11. Let $W^{\prime}$ be a $\widetilde{\mathbf{g}}$-submodule of $\mathbf{V}_{a}^{\kappa}$ such that $0 \neq W^{\prime} \neq \mathbf{V}_{a}^{\kappa}$. There exists $a^{\prime} \in \mathbf{N}^{I}$ such that $\frac{1}{2 \kappa}\left(\left\langle a^{\prime}, a^{\prime}+2\right\rangle-\langle a, a+2\rangle\right) \in\{1,2,3, \ldots\}$.

Let $a^{\prime}$ be as in the previous lemma applied with $W=0$ and $W^{\prime}$. Let $\lambda=\frac{1}{2 \kappa}\left(\left\langle a^{\prime}, a^{\prime}+2\right\rangle\right.$. Applying 2.7 (e) with $V^{\prime}=W^{\prime}$ (recall that $W^{\prime} \neq \mathbf{V}_{a}^{\kappa}$ ) we see that $\lambda-\frac{1}{2 \kappa}\langle a, a+2\rangle \in\{1,2,3, \ldots\}$. The lemma follows.

Proposition 2.12. (a) If $\kappa \notin \mathbf{Q}$, then $\mathbf{V}_{a}^{\kappa}$ is an irreducible $\tilde{\mathbf{g}}$-module for any $a \in \mathbf{N}^{I}$.

(b) If $\kappa \in \mathbf{Q}_{<0}$, then $\mathbf{V}_{a}^{\kappa}$ is an irreducible $\widetilde{\mathbf{g}}-$ module for any $a \in \mathbf{N}^{I}$ such that $\langle a, a+2\rangle<-2 \kappa$. In particular, $\mathbf{V}_{0}^{\kappa}$ is irreducible.

If $\mathbf{V}_{a}^{\kappa}$ is reducible, then from 2.11 we see that $\kappa$ is rational. If, in addition, we have $\kappa \in \mathbf{Q}_{<0}$, then 2.11 shows that $\langle a, a+2\rangle+2 \kappa n=\left\langle a^{\prime}, a^{\prime}+2\right\rangle$ for some integer $n \geq 1$. Since $\left\langle a^{\prime}, a^{\prime}+2\right\rangle=\sum b_{i, j} a^{\prime}(i)\left(a^{\prime}(j)+2\right)$ and the quantities $b_{i j}, a^{\prime}(i)$ are $\geq 0$, we have $\left\langle a^{\prime}, a^{\prime}+2\right\rangle \geq 0$. Hence, $\langle a, a+2\rangle+2 \kappa n \geq 0$. Since $\kappa<0$ and $n \geq 1$, it follows that $\langle a, a+2\rangle+2 \kappa \geq 0$. The proposition is proved. 
2.13. In the following result we will use the following notation: for $a \in \mathbf{N}^{I}, F_{a}$ denotes the finite set

$$
F_{a}=\left\{a^{\prime} \in \mathbf{N}^{I} \mid\left\langle a^{\prime}+1, a^{\prime}+1\right\rangle \leq\langle a+1, a+1\rangle\right\} .
$$

Proposition 2.14. Assume that $\kappa \notin \mathbf{Q}_{\geq 0}$. Let $a \in \mathbf{N}^{I}$. For any $\widetilde{\mathbf{g}}$-submodule $W$ of $\mathbf{V}_{a}^{\kappa}$ we denote $\delta(W)=\sum_{\lambda} \operatorname{dim}\left(W \cap \cap_{\lambda} \mathbf{V}_{a}^{\kappa}\right) \in \mathbf{N}$ where the sum is taken over all $\lambda \in \mathbf{C}$ such that $\lambda=\frac{1}{2 \kappa}\left\langle a^{\prime}, a^{\prime}+2\right\rangle$ for some $a^{\prime} \in F_{a}$.

(a) If $W, W^{\prime}$ are $\widetilde{\mathbf{g}}$-submodules of $\mathbf{V}_{a}^{\kappa}$ such that $W \subset W^{\prime}$ and $W \neq W^{\prime}$, then $\delta(W)<\delta\left(W^{\prime}\right)$.

(b) The $\widetilde{\mathbf{g}}$-module $\mathbf{V}_{a}^{\kappa}$ has finite length. All its composition factors are of the form $\mathbf{L}_{a^{\prime}}^{\kappa}$ for various $a^{\prime} \in F_{a}$.

(c) All composition factors of the unique maximal submodule of $\mathbf{V}_{a}^{\kappa}$ are of the form $\mathbf{L}_{a^{\prime}}^{\kappa}$ for various $a^{\prime} \in \mathbf{N}^{I}$ such that $\left\langle a^{\prime}+1, a^{\prime}+1\right\rangle\langle\langle a+1, a+1\rangle$. In particular, the simple module $\mathbf{L}_{a}^{\kappa}$ appears exactly once in a composition series of $\mathbf{V}_{a}^{\kappa}$.

In the setup of (a), we consider $a^{\prime}$ given by 2.10. Let $\lambda=\frac{1}{2 \kappa}\left\langle a^{\prime}, a^{\prime}+2\right\rangle$. If we had $\left\langle a^{\prime}, a^{\prime}+2\right\rangle-\langle a, a+2\rangle>0$, then from the inclusion

$$
\frac{1}{2 \kappa}\left(\left\langle a^{\prime}, a^{\prime}+2\right\rangle-\langle a, a+2\rangle\right) \in \mathbf{N}
$$

(see 2.10(b)) we would deduce that $\frac{1}{2 \kappa} \in \mathbf{Q}_{\geq 0}$, contradicting our assumption on $\kappa$. Thus, we must have the opposite inequality $\left\langle a^{\prime}, a^{\prime}+2\right\rangle-\langle a, a+2\rangle \leq 0$, which shows that $a^{\prime} \in F_{a}$.

By $2.10(\mathrm{a})$, the $\lambda$-eigenspace of $L_{0}: W^{\prime} / W \rightarrow W^{\prime} / W$ is nonzero. Hence, the dimension of the $\lambda$-eigenspace of $L_{0}: W^{\prime} \rightarrow W^{\prime}$ is strictly bigger than the dimension of the $\lambda$-eigenspace of $L_{0}: W \rightarrow W$. Using the definition we deduce that $\delta(W)<\delta\left(W^{\prime}\right)$ and (a) is proved.

From (a) it is clear that the $\widetilde{\mathbf{g}}$-module $\mathbf{V}_{a}^{\kappa}$ must be of finite length $\leq \delta\left(\mathbf{V}_{a}^{\kappa}\right)$.

Now let $W \subset W^{\prime}$ be $\widetilde{\mathbf{g}}$-submodules of $\mathbf{V}_{a}^{\kappa}$ such that $W^{\prime} / W$ is simple as a $\widetilde{\mathbf{g}}$-module. By the proof of 2.10 , there exists a nonzero homomorphism of $\widetilde{\mathbf{g}}$-modules $\mathbf{V}_{a^{\prime}}^{\kappa} \rightarrow W^{\prime} / W$. Since $W^{\prime} / W$ is simple, we must have $W^{\prime} / W \cong \mathbf{L}_{a^{\prime}}^{\kappa}$ and (b) is proved. (c) follows in the same way, using $2.7(\mathrm{~d})$. The proposition is proved.

In the remainder of this paper it is assumed that $\kappa \notin \mathbf{Q}_{\geq 0}$.

Definition 2.15. $\mathscr{O}_{\kappa}$ is the full subcategory of the category of $\tilde{\mathbf{g}}$-modules whose objects are the $\widetilde{\mathbf{g}}$-modules $V$ with the following properties:

(a) $V$ has central charge $\kappa-h$, and

(b) there exists a finite composition series of $V$ with all subquotients of the form $\mathbf{L}_{a}^{\kappa}$ for various $a \in \mathbf{N}^{I}$.

2.16. By 2.14(b), any Weyl module $\mathrm{V}_{a}^{\kappa}$ belongs to $\mathscr{O}_{\kappa}$. Using $2.5(\mathrm{a})$, we deduce that any generalized Weyl module $\mathscr{N}^{\kappa}$ belongs to $\mathscr{O}_{\kappa}$.

We have the following result. 
Proposition 2.17. If $V$ belongs to $\mathscr{O}_{\kappa}$, then $V$ is smooth and $\operatorname{dim} V(N)<\infty$ for all $N \geq 1$.

To prove that $\operatorname{dim} V(N)<\infty$ for $N \geq 1$, it is enough, by $1.10(\mathrm{e})$, to prove this only for $N=1$.

A Weyl module $\mathbf{V}_{a}^{\kappa}$ is smooth, by $2.7(\mathrm{f})$; hence its quotient $\mathbf{L}_{a}^{\kappa}$ is smooth. Note also that $\mathbf{L}_{a}^{\kappa}(1)$ is finite dimensional by 2.9. Using the definition of $\mathscr{O}_{\kappa}$, we are reduced to proving the following result.

Lemma 2.18. Let $0 \rightarrow V^{\prime} \rightarrow V \rightarrow V^{\prime \prime} \rightarrow 0$ be an exact sequence of $\tilde{\mathbf{g}}$-modules.

(a) If $V^{\prime}=V^{\prime}(\infty)$ and $V^{\prime \prime}=V^{\prime \prime}(\infty)$, then $V=V(\infty)$.

(b) We have an exact sequence $0 \rightarrow V^{\prime}(1) \rightarrow V(1) \rightarrow V^{\prime \prime}(1)$. Hence, if $\operatorname{dim} V^{\prime}(1)<\infty$ and $\operatorname{dim} V^{\prime \prime}(1)<\infty$, then $\operatorname{dim} V(1)<\infty$.

The proof of (b) is immediate. We now prove (a).

Let $B$ be a $\mathbf{C}$-basis of $\mathbf{g}$. Let $y \in V$ and let $y^{\prime \prime}$ be the image of $y$ in $V^{\prime \prime}$. By assumption, we have $y^{\prime \prime} \in V^{\prime \prime}(N)$ for some $N \geq 1$. Hence, the vectors $\left(\epsilon c_{1}\right) \cdots\left(\epsilon c_{N}\right)(y)$ belong to $V^{\prime}$ for any sequence $c_{1}, \ldots, c_{N}$ in $B$. Since there are only finitely many such vectors ( $N$ is fixed), they are all contained in $V^{\prime}\left(N^{\prime}\right)$ for some $N^{\prime} \geq 1$ (by our assumption on $V^{\prime}$ ).

Thus, for any $c_{1}^{\prime}, \ldots, c_{N^{\prime}}^{\prime}$ in $B$ and any $c_{1}, \ldots, c_{N}$ in $B$ we have

$$
\left(\epsilon c_{1}^{\prime}\right) \cdots\left(\epsilon c_{N^{\prime}}^{\prime}\right)\left(\epsilon c_{1}\right) \cdots\left(\epsilon c_{N}\right)(y)=0
$$

so that $y \in V\left(N+N^{\prime}\right)$. This completes the proof of the lemma and, hence, that of 2.17 .

2.19. Let $V$ be an object of $\mathscr{O}_{\kappa}$. For any $\lambda \in \mathbf{C}$ we denote by ${ }_{\lambda} V$ the $\mathbf{C}$ subspace of $V$ consisting of all $x \in V$ such that $x$ is in the kernel of some power of $\left(L_{0}-\lambda I\right): V \rightarrow V$ where $L_{0}$ is the Sugawara operator.

Lemma 2.20. (a) We have $V=\bigoplus_{\lambda \in \mathbf{C}}\left({ }_{\lambda} V\right)$ and, for any $\lambda \in \mathbf{C}$, we have $\operatorname{dim}_{\lambda} V<\infty$.

(b) There exist $l_{1}, \ldots, l_{m}$ in $\mathbf{N}$ such that

$$
\left\{\left.\lambda \in \mathbf{C}\right|_{\lambda} V \neq 0\right\} \subset\left\{\frac{l_{1}}{2 \kappa}+\mathbf{N}\right\} \cup \cdots \cup\left\{\frac{l_{m}}{2 \kappa}+\mathbf{N}\right\} .
$$

The lemma is already known in the case where $V$ is a Weyl module. (See 2.7.) If the lemma is true for $V$ then it is clearly true for a quotient $\widetilde{\mathbf{g}}$-module of $V$. Therefore, it is true for the simple quotient of a Weyl module. If the lemma is true for a $\widetilde{\mathbf{g}}$-submodule of $V$ and for the corresponding quotient, then it is clearly true for $V$ itself. We apply this repeatedly to a composition series of $V$ and the lemma follows.

We shall write $\Re z$ for the real part of a complex number $z$.

Proposition 2.21. Let $V$ be an object of $\mathscr{O}_{\kappa}$.

(a) For any $\lambda \in \mathrm{C}$ and any $N \geq 0$ we have $Q_{N}^{\sharp}\left({ }_{\lambda} V\right) \subset{ }_{\lambda+N} V$.

(b) For any real number $t$ there exists $N \geq 1$ such that $\bigoplus_{\Re \lambda \geq t}\left({ }_{\lambda} V\right) \supset Q_{N}^{\sharp} V$. 
(c) Consider the C-linear map $\phi_{\lambda}: \mathbf{g} \otimes{ }_{\lambda} V \rightarrow{ }_{\lambda+1} V$ given by $c \otimes v \mapsto\left(\epsilon^{-1} c\right) v$ (see (a)). There exists a real number $t^{\prime}$ such that $\phi_{\lambda}$ is surjective for all $\lambda \in \mathbf{C}$ such that $\Re \lambda \geq t^{\prime}$.

(d) For any $N \geq 1$ there exists a real number $t^{\prime \prime}$ such that $\bigoplus_{\Re \lambda \geq t^{\prime \prime}}\left({ }_{\lambda} V\right) \subset$ $Q_{N}^{\sharp} V$.

(e) For any $N \geq 0$, the $\mathbf{C}$-vector space $V / Q_{N}^{\sharp} V$ is finite dimensional.

In the case where $N=0,(\mathrm{a})$ is trivial. The case where $N \geq 1$ can be immediately reduced to the case where $N=1$; in that case the result follows from the commutation formulas 1.14(b) for Sugawara operators.

We prove (b). Let $t \in \mathbf{R}$. Let $l_{1}, \ldots, l_{m}$ be as in 2.20. Let $N \geq 1$ be such that $N \geq \max _{u}\left(t-\Re\left(\frac{1}{2 \kappa}\right) l_{u}\right)$. Let $\lambda$ be such that ${ }_{\lambda} V \neq 0$; by the definition of the $l_{u}$, we have $\lambda=\frac{1}{2 \kappa} l_{u}+n$ for some $n \geq 0$ and some $u$.

By (a), we have $Q_{N \lambda}^{\sharp} V \subset_{\lambda+N} V$, and it remains to show that $\Re(\lambda+N) \geq t$ or that $\Re\left(\frac{1}{2 \kappa}\right) l_{u}+n+N \geq t$. This holds by the definition of $N$. Thus, (b) is proved.

We now prove (c).

Assume that $0 \rightarrow V^{\prime} \rightarrow V \rightarrow V^{\prime \prime} \rightarrow 0$ is an exact sequence in $\mathscr{O}_{\kappa}$. Then from 2.20(a) we see that for any $\lambda \in \mathbf{C}$ we have an induced exact sequence $0 \rightarrow{ }_{\lambda} V^{\prime} \rightarrow{ }_{\lambda} V \rightarrow{ }_{\lambda} V \rightarrow 0$. Tensoring with $\mathbf{g}$ we obtain an exact sequence that is the top row of the following commutative diagram with exact rows

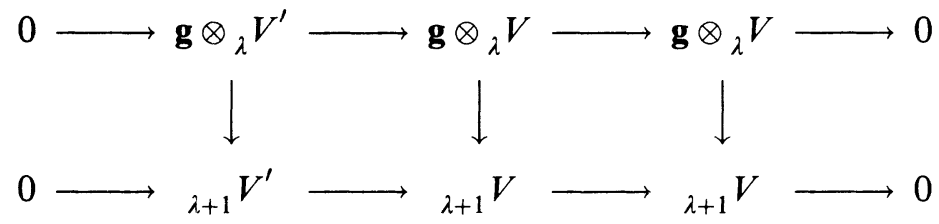

where the vertical maps are as in (c).

From this we see that if (c) holds for $V^{\prime}$ and for $V^{\prime \prime}$, then it also holds for $V$; moreover, if (c) holds for $V$, then it also holds for $V^{\prime \prime}$.

Thus we are first reduced to the case where $V$ is a simple object of $\mathscr{O}_{K}$ and then we are further reduced to the case where $V$ is a Weyl module $\mathbf{V}_{a}^{\kappa}$.

Let $\lambda_{0}=\frac{1}{2 \kappa}\langle a, a+2\rangle$. Then $\lambda_{0} \mathbf{V}_{a}^{\kappa}=\mathscr{V}_{a}$ (see 2.7(d).) By the definition of Weyl modules we have $\sum_{N \geq 0} Q_{N}^{\sharp} \mathscr{V}_{a}=\mathbf{V}_{a}^{\kappa}$. By (a) we have $Q_{N}^{\sharp} \mathscr{V}_{a} \subset{ }_{\lambda_{0}+N} \mathbf{V}_{a}^{\kappa}$; this implies in view of the previous equality and 2.20(a) that $Q_{N}^{\sharp} \mathscr{V}_{a}={ }_{\lambda_{0}+N} \mathbf{V}_{a}^{\kappa}$ for all $N \geq 0$. This shows that (c) holds with $t^{\prime}=\Re \lambda_{0}$.

This completes the proof of (c).

By (c), for any $N \geq 1$ and any $\lambda \in \mathbf{C}$ such that $\Re \lambda \geq t^{\prime}+N$, we have that $Q_{N \lambda-N}^{\sharp} V={ }_{\lambda} V$. This clearly implies (d).

We prove (e). Using (d) and 2.20(a) we see that, for any $N$, there exists a real number $t^{\prime \prime}$ such that the natural map $\bigoplus_{\Re \lambda<t^{\prime \prime}}\left({ }_{\lambda} V\right) \rightarrow V / Q_{N}^{\sharp} V$ is surjective. The last direct sum is a finite-dimensional $\mathbf{C}$-vector space by 2.20 . It follows that $V / Q_{N}^{\sharp} V$ is a finite-dimensional $\mathbf{C}$-vector space. The proposition is proved. 
Theorem 2.22. Let $V$ be a $\widetilde{\mathbf{g}}$-module with central charge $\kappa-h$. The following conditions are equivalent.

(a) $V$ is in $\mathscr{O}_{\kappa}$.

(b) $V$ is a quotient of a generalized Weyl module.

(c) There exists $N \geq 1$ such that $V(N)$ is finite dimensional and generates $V$ as a $\widetilde{\mathbf{g}}$-module.

If $V$ satisfies (c), then it satisfies (b), by $2.5(\mathrm{~b})$. If $V$ satisfies (b), then it satisfies (a), by 2.16. (A quotient of an object in $\mathscr{O}_{\kappa}$ is in $\mathscr{O}_{\kappa}$. )

Now let $V$ be an object in $\mathscr{O}_{\kappa}$. We will show that $V$ satisfies (c). Let $M=\bigoplus_{\Re \lambda<t^{\prime}+1}\left({ }_{\lambda} V\right)$ where $t^{\prime}$ is as in $2.21(\mathrm{c})$. This is a finite-dimensional Csubspace of $V$ by 2.20 .

We show by induction on $N \geq 0$ that

$$
{ }_{\lambda} V \subset Q_{N}^{\sharp} M \text { for any } \lambda \in \mathbf{C} \text { such that } t^{\prime}+N \leq \Re \lambda<t^{\prime}+N+1 .
$$

For $N=0$ this follows from the definition of $M$. Assume now that $N \geq 1$ and that our assertion is known for $N-1$. If $t^{\prime}+N \leq \Re \lambda<t^{\prime}+N+1$, then by 2.21 (c) we have ${ }_{\lambda} V \subset Q_{1}^{\sharp}\left({ }_{\lambda-1} V\right)$ and by the induction hypothesis we have $Q_{1}^{\sharp}\left({ }_{\lambda-1} V\right) \subset Q_{1}^{\sharp} Q_{N-1}^{\sharp} M \subset Q_{N}^{\sharp} M$. This proves our assertion by induction. It implies, using the definition of $M$ and 2.20, that $V=\sum_{N \geq 0} Q_{N}^{\sharp} M$. Thus, $M$ generates $V$ as a $\widetilde{\mathbf{g}}$-module. Since $V$ is smooth (2.17) and $M$ is finite dimensional, we have $M \subset V(N)$ for some $N \geq 1$. This $V(N)$ will then generate $V$ as a $\widetilde{\mathbf{g}}$-module; it is finite dimensional, by 2.17 . We have proved that $V$ satisfies (c). The theorem is proved.

2.23. We now study the $\widetilde{\mathbf{g}}$-module $D(V)$ (see 1.16) for $V$ in $\mathscr{G}_{\kappa}$. As a vector space, $D(V)$ consists of those $\mathbf{C}$-linear forms $f: V \rightarrow \mathbf{C}$ such that $f$ is zero on $Q_{N}^{\sharp} V$ for some $N \geq 1$. By $2.21(\mathrm{c})$, (d), this condition on $f$ is equivalent to the condition that $f$ is zero on $\left.\bigoplus_{\Re \lambda \geq t}{ }_{\lambda} V\right)$ for some $t \in \mathbf{R}$ and, by 2.20, this is equivalent to the condition that $f$ is zero on all but finitely many of the subspaces ${ }_{\lambda} V$ of $V$. Thus, as a $\mathrm{C}$-vector space, we have

$$
D(V)=\bigoplus_{\lambda}^{d}\left({ }_{\lambda} V\right) \text {. }
$$

From the definition, $D(V)$ is a smooth $\widetilde{\mathbf{g}}$-module with central charge $\kappa-h$. Hence the Sugawara operators are well defined on $D(V)$. From 1.16(b) we see that the subspace ${ }^{d}\left({ }_{\lambda} V\right)$ of $D(V)$ is precisely the set of all vectors in $D(V)$ annihilated by some power of $L_{0}-\lambda: D(V) \rightarrow D(V)$; we denote it ${ }_{\lambda} D(V)$.

Proposition 2.24. If $V$ is the $\tilde{\mathbf{g}}$-module $\mathbf{L}_{a}^{\kappa}$, then the $\widetilde{\mathbf{g}}$-module $D(V)$ is isomorphic to $\mathbf{L}_{\bar{a}}^{\kappa}$ where $\bar{a}$ is as in 1.2.

Assume that $W$ is a $\widetilde{\mathbf{g}}$-submodule of $D(V)$ distinct from 0 and $D(V)$. Then $W$ is stable under $L_{0}: D(V) \rightarrow D(V)$ and the direct sum decomposition $D(V)=\bigoplus_{\lambda}\left({ }_{\lambda} D(V)\right)$ (see 2.23) induces a direct sum decomposition $W=\bigoplus_{\lambda}\left(W \cap{ }_{\lambda} D(V)\right)$. Since $W \neq 0$ and $W \neq D(V)$, we must have 
(a)

$$
W \cap_{\lambda} D(V) \neq 0 \text { and } W \cap_{\lambda^{\prime}} D(V) \neq{ }_{\lambda^{\prime}} D(V)
$$

for some $\lambda, \lambda^{\prime}$.

Let $W^{\perp}=\{x \in V \mid f(x)=0 \quad \forall f \in W\}$. Then $W^{\perp}$ is clearly a $\widetilde{\mathbf{g}}$-submodule of $V$ and from (a) we see that $W^{\perp}$ is distinct from 0 and $V$. This contradicts the fact that $V$ is simple. Thus, $D(V)$ is a simple $\widetilde{\mathbf{g}}$-module.

Let $\lambda_{0}=\frac{1}{2 \kappa}\langle a, a+2\rangle$. By 2.7, we have ${ }_{\lambda} V=0$ unless $\lambda-\lambda_{0} \in \mathbf{N}$. By 2.23, we then have ${ }_{\lambda} D(V)=0$ unless $\lambda-\lambda_{0} \in \mathbf{N}$. For any $n \geq 1$ and $c \in \mathbf{g}$, we have $\left(\epsilon^{n} c\right)_{\lambda_{0}} D(V) \subset{ }_{\lambda_{0}-n} D(V)$ by $1.14(\mathrm{~b})$ and, hence, $\left(\epsilon^{n} c\right)_{\lambda_{0}} D(V)=0$. Thus, the finite-dimensional $\mathbf{g}$-stable subspace ${ }_{\lambda_{0}} D(V)$, is annihilated by $(\epsilon \mathbf{C}[\epsilon]) \otimes \mathbf{g}$; hence, there exists a $\widetilde{\mathbf{g}}$-module homomorphism $\mathbf{V}_{a^{\prime}}^{\kappa} \rightarrow D(V)$ that maps $\mathscr{V}_{a^{\prime}}$ injectively into ${ }_{\lambda_{0}} D(V)$. Since $D(V)$ is simple, this must define an isomorphism of $\widetilde{\mathbf{g}}$-modules $\mathbf{L}_{a^{\prime}}^{\kappa} \cong D(V)$. As a $\mathbf{g}$-module, ${ }_{\lambda_{0}} D(V)$ is isomorphic to the dual of $\lambda_{0} V$ and, hence, to the dual of $\mathscr{V}_{a}$; in particular, it is isomorphic to $\mathscr{V}_{\bar{a}}$. It follows that $a^{\prime}=\bar{a}$. The proposition is proved.

Proposition 2.25. (a) If $0 \rightarrow V^{\prime} \rightarrow V \rightarrow V^{\prime \prime} \rightarrow 0$ is an exact sequence in $\mathscr{O}_{\kappa}$, then the corresponding sequence of $\widetilde{\mathbf{g}}$-modules $0 \rightarrow D\left(V^{\prime \prime}\right) \rightarrow D(V) \rightarrow D\left(V^{\prime}\right) \stackrel{\kappa}{\rightarrow}$ 0 is exact.

(b) If $V$ belongs to $\mathscr{O}_{\kappa}$, then the $\tilde{\mathbf{g}}$-module $D(V)$ belongs to $\mathscr{O}_{\kappa}$.

We first prove (a). From 2.20 it follows that for any $\lambda \in \mathbf{C}$ we have an induced exact sequence of $\mathbf{C}$-vector spaces $0 \rightarrow{ }_{\lambda} V^{\prime} \rightarrow{ }_{\lambda} V \rightarrow{ }_{\lambda} V \rightarrow 0$.

Taking dual spaces we obtain an exact sequence $0 \rightarrow{ }^{d}\left({ }_{\lambda} V^{\prime \prime}\right) \rightarrow{ }^{d}\left({ }_{\lambda} V\right) \rightarrow$ ${ }^{d}\left({ }_{\lambda} V^{\prime}\right) \rightarrow 0$.

Taking the direct sum over all $\lambda$ and using 2.23(a), we obtain an exact sequence $0 \rightarrow D\left(V^{\prime \prime}\right) \rightarrow D(V) \rightarrow D\left(V^{\prime}\right) \rightarrow 0$. This proves (a).

To prove (b) we see, by repeated application of (a), that we are reduced to the case where $V$ is simple. In that case, we may use 2.24 . The proposition is proved.

Proposition 2.26. If $V$ is in $\mathscr{O}_{\kappa}$, then the natural map $V \rightarrow D(D(V)$ ) (see $1.16(\mathbf{a}))$ is an isomorphism of $\widetilde{\mathbf{g}}$-modules.

With the notation in 2.23 we have

$$
{ }_{\lambda} D(V) \cong{ }^{d}\left({ }_{\lambda} V\right) .
$$

Applying (a) to $D(V)$ instead of $V$, we obtain

$$
\left.{ }_{\lambda} D(D(V)) \cong{ }^{d}{ }_{\lambda} D(V)\right) .
$$

Taking the dual of (a) (in which the vector spaces involved are finite dimensional), we obtain

$$
\left.{ }_{\lambda} V \cong{ }_{\lambda}^{d} D(V)\right) .
$$

From (b) and (c), we obtain ${ }_{\lambda} V \cong{ }_{\lambda} D(D(V))$. Taking the direct sum over $\lambda$, we obtain $V \cong D(D(V))$; it is clear that this coincides with the map 1.16(a). The proposition is proved. 
2.27. Now assume that we are given a set $\odot$ with two elements $s, s^{\prime}$. Let $V_{s}, V_{s^{\prime}}$ be objects of $\mathscr{O}_{\kappa}$. Then $V=V_{s} \otimes V_{s^{\prime}}$ is a $\widetilde{\mathbf{g}}^{\mathcal{O}}$-module as in 1.13.

Proposition 2.28. (a) $V$ is in $\mathscr{O}_{\kappa}$ (relative to $\widetilde{\mathbf{g}}^{\circ}$ ).

(b) $D(V)=D\left(V_{s}\right) \otimes D\left(V_{s^{\prime}}\right)$.

(a) follows, for example, from the characterization $2.22\left(\right.$ b) of $\mathscr{O}_{\kappa}$.

We prove (b). From the definitions we see that for any $\lambda \in \mathbf{C}$ we have

$$
{ }_{\lambda} V=\bigoplus_{\lambda_{1}+\lambda_{2}=\lambda}\left({ }_{\lambda_{1}} V_{s} \otimes_{\lambda_{2}} V_{s^{\prime}}\right)
$$

(finite sum). Taking dual spaces for both sides (which are finite dimensional) we obtain

$$
{ }_{\lambda}^{d} V=\bigoplus_{\lambda_{1}+\lambda_{2}=\lambda}\left({ }^{d}\left({ }_{\lambda_{1}} V_{s}\right) \otimes{ }^{d}\left({ }_{\lambda_{2}} V_{s^{\prime}}\right)\right) .
$$

Taking now the direct sum over $\lambda$, we obtain (b).

Proposition 2.29. Let $V, V^{\prime}$ be two objects of $\mathscr{O}_{\kappa}$. The C-vector space $\operatorname{Hom}_{\mathscr{O}_{\kappa}}\left(V, V^{\prime}\right)$ is finite dimensional.

If $V_{1} \rightarrow V$ is a surjective homomorphism of $\widetilde{\mathbf{g}}$-modules, we have a natural imbedding $\operatorname{Hom}_{\mathscr{O}_{\kappa}}\left(V, V^{\prime}\right) \rightarrow \operatorname{Hom}_{\mathscr{O}_{\kappa}}\left(V_{1}, V^{\prime}\right)$. Thus our assertion for $\left(V, V^{\prime}\right)$ follows from the assertion for $\left(V_{1}, V^{\prime}\right)$. By 2.22, we can find a surjective homomorphism of $\tilde{\mathbf{g}}$-modules $V_{1} \rightarrow V$ with $V_{1}$ a generalized Weyl module. Thus we are reduced to the case where $V$ is a generalized Weyl module $\mathscr{N}^{\kappa}$. By 2.3(a), we are then reduced to showing that $\operatorname{dim} \operatorname{Hom}_{\mathrm{g}[\epsilon]}\left(\mathscr{N}, V^{\prime}\right)<\infty$. By the definition of a nil-module, there exists $t \geq 1$ such that the image of any $\mathbf{g}[\epsilon]$-homomorphism $\mathscr{N} \rightarrow V^{\prime}$ is contained in $V^{\prime}(t)$. Thus, $\operatorname{Hom}_{\mathrm{g}[\epsilon]}\left(\mathscr{N}, V^{\prime}\right)=$ $\operatorname{Hom}_{\mathrm{g}[\epsilon]}\left(\mathcal{N}, V^{\prime}(t)\right)$. Since $\mathcal{N}$ and $V^{\prime}(t)$ are finite dimensional, the proposition follows.

2.30. Let $V, V^{\prime}$ be two objects of $\mathscr{O}_{\kappa}$. Let $W$ be the subspace of $\prod_{\lambda, \lambda^{\prime}} \operatorname{Hom}\left({ }_{\lambda} V \otimes_{\lambda^{\prime}} V^{\prime}, \mathbf{C}\right)$ consisting of all $f_{\lambda, \lambda^{\prime}}$ such that

$$
f_{\lambda, \lambda^{\prime}}\left(\left(\epsilon^{n} c\right) x \otimes y\right)+f_{\lambda+n, \lambda^{\prime}+n}\left(x \otimes\left((-\epsilon)^{-n} c\right) y\right)=0
$$

for all $\lambda, \lambda^{\prime} \in \mathbf{C}, c \in \mathbf{g}, n \in \mathbf{Z}$ and all $x \in{ }_{\lambda+n} V, y \in \epsilon_{\lambda^{\prime}} V^{\prime}$. Let $W_{1}$ be the subspace of $W$ consisting of those $\left(f_{\lambda, \lambda^{\prime}}\right)$ such that for any $\lambda \in \mathbf{C}$, we have $f_{\lambda, \lambda^{\prime}}=0$ for all but finitely many $\lambda^{\prime}$.

Using 2.20 and 2.23, we see that $\operatorname{Hom}_{\mathscr{O}_{\kappa}}\left(V, D\left(V^{\prime}\right)\right)$ may be naturally identified with the subspace $W_{1}$ of

$$
\prod_{\lambda, \lambda^{\prime}} \operatorname{Hom}\left({ }_{\lambda} V,{ }_{\lambda^{\prime}} D\left(V^{\prime}\right)\right)=\prod_{\lambda, \lambda^{\prime}} \operatorname{Hom}\left({ }_{\lambda} V \otimes{ }_{\lambda^{\prime}} V^{\prime}, \mathbf{C}\right)=W .
$$

On the other hand, we may regard $V \otimes V^{\prime}$ as a $\widetilde{\mathbf{g}}$-module with zero action of 1 and with

$$
\left(\epsilon^{n} c\right)(x \otimes y)=\left(\left(\epsilon^{n} c\right) x\right) \otimes y+x \otimes\left((-\epsilon)^{-n} c\right)(y)
$$

for all $c \in \mathbf{g}, n \in \mathbf{Z}, x \in V, y \in V^{\prime}$. Using again 2.20, it is clear that the 
vector space $\operatorname{Hom}_{\tilde{\mathbf{g}}}\left(V \otimes V^{\prime}, \mathbf{C}\right.$ ) (where $\mathbf{C}$ is taken with zero $\widetilde{\mathbf{g}}$-action) may be naturally identified with $W$. The imbedding $W_{1} \subset W$ may therefore be considered as a linear map

$$
\operatorname{Hom}_{\mathscr{O}_{\kappa}}\left(V, D\left(V^{\prime}\right)\right) \rightarrow \operatorname{Hom}_{\widetilde{\mathbf{g}}}\left(V \otimes V^{\prime}, \mathbf{C}\right) .
$$

Proposition 2.31. The linear map 2.30(b) is an isomorphism.

We must prove that $W=W_{1}$. Let $\lambda \in \mathbf{C}$. Since ${ }_{\lambda} V$ is finite dimensional, we can find $N \geq 1$ such that ${ }_{\lambda} V \subset V(N)$. By $2.21(\mathrm{~d})$, we can find $t>0$ such that for any $\lambda^{\prime}$ satisfying $\Re \lambda^{\prime} \geq t$ we have $\lambda^{\prime} V^{\prime} \subset Q_{N}^{\sharp} V^{\prime}$. Consider $y \in{ }_{\lambda^{\prime}} V^{\prime}$ for such $\lambda^{\prime}$. Then we can write $y$ as a sum of elements of form $\left(\epsilon^{-1} c_{1}\right) \cdots\left(\epsilon^{-1} c_{N}\right) z$ with $z \in_{\lambda^{\prime}-N} V^{\prime}$. For any $x \in{ }_{\lambda} V$ we have

$$
\begin{aligned}
f_{\lambda, \lambda^{\prime}}(x \otimes y) & =\sum f_{\lambda, \lambda^{\prime}}\left(x \otimes\left(\epsilon^{-1} c_{1}\right) \cdots\left(\epsilon^{-1} c_{N}\right) z\right) \\
& =\sum f_{\lambda-N, \lambda^{\prime}-N}\left(\left(\epsilon c_{N}\right) \cdots\left(\epsilon c_{1}\right) x \otimes z\right)
\end{aligned}
$$

where we have used $N$ times 2.30(a) for $n=1$. But the last sum is zero since $x \in V(N)$. Thus we have $f_{\lambda, \lambda^{\prime}}=0$ unless $\Re \lambda^{\prime}<t$. By 2.20 , there are only finitely many eigenvalues $\lambda^{\prime}$ of $L_{0}: V^{\prime} \rightarrow V^{\prime}$ satisfying $\Re \lambda^{\prime}<t$. Thus we have $W_{1}=W$ and the proposition is proved.

2.32. To $V, V^{\prime}$ in $\mathscr{O}_{\kappa}$ we associate the $\mathbf{C}$-vector space

$$
\left\langle V, V^{\prime}\right\rangle=\left(V \otimes V^{\prime}\right) / \widetilde{\mathbf{g}}\left(V \otimes V^{\prime}\right)
$$

where $V \otimes V^{\prime}$ is regarded as a $\widetilde{\mathbf{g}}$-module as in 2.30 . (Thus this is not the standard tensor product of $\widetilde{\mathbf{g}}$-modules.)

It is clear that the C-linear isomorphism $V \otimes V^{\prime} \cong V^{\prime} \otimes V$ given by $x \otimes y \mapsto$ $y \otimes x$ preserves the images of the $\widetilde{\mathbf{g}}$-module actions and induces an isomorphism of $\mathbf{C}$-vector spaces

$$
\left\langle V, V^{\prime}\right\rangle=\left\langle V^{\prime}, V\right\rangle .
$$

The isomorphism $2.30(\mathrm{~b})$ asserted in 2.31 can be regarded as an isomorphism

$$
\operatorname{Hom}_{\mathscr{O}_{\kappa}}\left(V, D\left(V^{\prime}\right)\right) \cong \operatorname{Hom}_{\mathbf{C}}\left(\left\langle V, V^{\prime}\right\rangle, \mathbf{C}\right) \text {. }
$$

This, together with 2.29 , implies that $\operatorname{dim}\left\langle V, V^{\prime}\right\rangle<\infty$. Note that $V, V^{\prime} \mapsto$ $\left\langle V, V^{\prime}\right\rangle$ is naturally a functor from $\mathscr{O}_{\kappa} \times \mathscr{O}_{\kappa}$ to finite-dimensional vector spaces.

Proposition 2.33. (a) Any nonzero $\widetilde{\mathbf{g}}$-submodule of $D\left(\mathbf{V}_{a}^{\kappa}\right)$ contains the image of $D\left(\mathbf{L}_{a}^{\kappa}\right) \subset D\left(\mathbf{V}_{a}^{\kappa}\right)($ see $2.25(\mathbf{a}))$.

(b) $D\left(\mathbf{V}_{a}^{\kappa}\right)(1)$ is isomorphic to $\mathscr{V}_{\bar{a}}$ as a $\mathbf{g}$-module.

(a) is dual to the statement that $\mathbf{V}_{a}^{\kappa}$ has a unique maximal submodule, namely the kernel of $\mathbf{V}_{a}^{\kappa} \rightarrow \mathbf{L}_{a}^{\kappa}$.

We now prove (b). Let $\mathscr{V}^{\prime}$ be an irreducible $\mathbf{g}$-submodule of $D\left(\mathbf{V}_{a}^{\kappa}\right)(1)$. As in the proof of 2.9, we can find a homomorphism of $\widetilde{\mathbf{g}}$-modules $\phi$ : $\mathbf{V}_{b}^{\kappa} \rightarrow D\left(\mathbf{V}_{a}^{\kappa}\right)$ that carries $\mathscr{V}_{b}$ isomorphically onto $\mathscr{V}^{\prime}$. Since $\mathbf{L}_{b}^{\kappa}$ is a quotient 
of $\phi\left(\mathbf{V}_{b}^{\kappa}\right)$, it is a composition factor of $D\left(\mathbf{V}_{a}^{\kappa}\right)$. By duality, $\mathbf{L}_{b}^{\kappa}$ is a composition factor of $\mathbf{V}_{a}^{\kappa}$ (see 2.24); hence,

(c) $\frac{1}{2 \kappa}(\langle b, b+2\rangle-\langle a, a+2\rangle) \in \mathbf{N}$

(see 2.7). By (a), $\phi\left(\mathbf{V}_{b}^{\kappa}\right)$ contains the submodule $D\left(\mathbf{L}_{a}^{\kappa}\right)$. Assume that it contains it strictly. Then $D\left(\mathbf{L}_{a}^{\kappa}\right)$ is a composition factor of the maximal submodule of $\mathbf{V}_{b}^{\kappa}$; hence, again by 2.24 and 2.7 , we have

$$
\frac{1}{2 \kappa}(\langle a, a+2\rangle-\langle b, b+2\rangle) \in\{1,2,3, \ldots,\} .
$$

This contradicts (c). Thus, we must have $\phi\left(\mathbf{V}_{b}^{\kappa}\right)=D\left(\mathbf{L}_{a}^{\kappa}\right)$. This implies that $\mathscr{V}^{\prime} \subset D\left(\mathbf{L}_{a}^{\kappa}\right)(1)$. We now use 2.9; the proposition follows.

\section{A characterization of $\mathscr{O}_{\kappa}$}

3.1. In this section we will give a criterion for a $\widetilde{\mathbf{g}}$-module to be in $\mathscr{O}_{\kappa}$ by a condition which is weaker than that in $2.22(\mathrm{c})$. This criterion will be crucial in our study of tensor products.

Theorem 3.2. Let $V$ be a $\tilde{\mathbf{g}}$-module with central charge $\kappa-h$. The following conditions are equivalent.

(a) $V$ is in $\mathscr{O}_{\kappa}$.

(b) $V$ is smooth and $\operatorname{dim} V(1)<\infty$.

The proof will occupy the rest of this section. One of the main ingredients in the proof is the use of projective objects in (a truncation of) $\mathscr{O}_{\kappa}$. Soergel [So] has proved an abstract version of the Brauer (or BGG) reciprocity which is well adapted to our needs. In the subsections 3.3-3.7 we will show that Soergel's axioms hold in the case of interest to us. (Alternatively, one could use [RW].)

3.3. Let $t$ be an integer $\geq 1$. We consider the full subcategory $\mathscr{O}_{\kappa}^{t}$ of $\mathscr{O}_{\kappa}$ whose objects are the $V$ in $\mathscr{O}_{\kappa}$ with the following property: all composition factors of $V$ are of form $\mathbf{L}_{a}^{\kappa}$ for some $a$ in the finite set

$$
F^{t}=\left\{a \in \mathbf{N}^{I} \mid\langle a, a+2\rangle \leq t\right\} .
$$

We have $\mathscr{O}_{\kappa}^{t^{\prime}} \subset \mathscr{O}_{\kappa}^{t}$ if $t^{\prime} \leq t$. Clearly, any $V$ in $\mathscr{O}_{\kappa}$ belongs to $\mathscr{O}_{\kappa}^{t}$ for some

We regard $F^{t}$ as a partially ordered set: we say that $a^{\prime} \leq a$ if either $a^{\prime}=a$ or $\left\langle a^{\prime}, a^{\prime}+2\right\rangle<\langle a, a+2\rangle$.

The following properties are obvious.

(a) $\mathscr{O}_{\kappa}^{t}$ is closed under extensions in the category of $\widetilde{\mathbf{g}}$-modules with central charge $\kappa-h$.

$$
\text { If } V \text { is in } \mathscr{O}_{\kappa}^{t} \text {, then } D(V) \text { is in } \mathscr{O}_{\kappa}^{t} \text {. }
$$

Lemma 3.4. If $a \in F^{t}$, then $\mathbf{V}_{a}^{\kappa}$ is in $\mathscr{O}_{\kappa}^{t}$. Moreover, all composition factors of $\mathbf{V}_{a}^{\kappa}$ other than $\mathbf{L}_{a}^{\kappa}$ are of form $\mathbf{L}_{a^{\prime}}^{\kappa}$ with $a^{\prime} \in F^{t}, a^{\prime}<a$.

This follows from $2.14(\mathrm{c})$. 
Lemma 3.5. If $a$ is a maximal element of $F^{t}$ for $\leq$, then the canonical map $\mathbf{V}_{a}^{\kappa} \rightarrow \mathbf{L}_{a}^{\kappa}$ is a projective cover for $\mathbf{L}_{a}^{\kappa}$ in $\mathscr{O}_{\kappa}^{t}$.

If $f: \mathbf{V}_{a}^{\kappa} \rightarrow X$ is a morphism in $\mathscr{O}_{\kappa}^{t}$, then $f$ restricts to a $\mathbf{g}$-morphism from $\mathscr{V}_{a}$ into the (generalized) $\frac{1}{2 \kappa}\langle a, a+2\rangle$-eigenspace $\mathscr{E}_{X}$ of $L_{0}: X \rightarrow X$. Then $\frac{1}{2 \kappa}\langle a, a+2\rangle-1$ is not an eigenvalue of $L_{0}: X \rightarrow X$ (by the choice of $a$ ). An argument in the proof of 2.10 shows that $\mathscr{E}_{X}$ is contained in $X(1)$. Hence, restriction defines a bijection between $\widetilde{\mathbf{g}}$-morphisms $\mathbf{V}_{a}^{\kappa} \rightarrow X$ and $\mathbf{g}$-morphisms from $\mathscr{V}_{a}$ to $\mathscr{E}_{X}$.

Now let $p: X^{\prime} \rightarrow X$ be a surjective morphism in $\mathscr{O}_{\kappa}^{t}$ and let $f: \mathbf{V}_{a}^{\kappa} \rightarrow X$ be any morphism in $\mathscr{O}_{\kappa}^{t}$. We want to lift $f$ to a morphism $f^{\prime}: \mathbf{V}_{a}^{\kappa} \rightarrow X^{\prime}$ such that $p f^{\prime}=f$. Our morphisms restrict to g-morphisms $p_{1}: \mathscr{E}_{X^{\prime}} \rightarrow \mathscr{E}_{X}$ and $f_{1}: \mathscr{V}_{a} \rightarrow \mathscr{E}_{X}$ with $p_{1}$ surjective. But in the category of finite-dimensional g-modules, all objects are projective. Hence, we can find a g-morphism $f_{1}^{\prime}$ : $\mathscr{V}_{a} \rightarrow \mathscr{E}_{X^{\prime}}$ such that $p_{1} f_{1}^{\prime}=f_{1}$. By the earlier argument, $f_{1}^{\prime}$ extends uniquely to a $\widetilde{\mathbf{g}}$-module homomorphism $f^{\prime}: \mathbf{V}_{a}^{\kappa} \rightarrow X^{\prime}$. This clearly has the required property. Thus we have proved that $\mathbf{V}_{a}^{\kappa}$ is projective in $\mathscr{O}_{\kappa}^{t}$. It is clearly an indecomposable object. The lemma is proved.

Lemma 3.6. For any $a \in \mathbf{N}^{I}$, the endomorphisms of the $\tilde{\mathbf{g}}$-module $V=\mathbf{L}_{a}^{\kappa}$ are just the scalars in $\mathbf{C}$.

Indeed such an endomorphism is completely determined by its restriction to $V(1)=\mathscr{V}_{a}$, and then one uses Schur's lemma for simple g-modules.

3.7. Let $\mathscr{C}$ (resp. $\mathscr{C}^{+}, \mathscr{C}^{0}$ ) be the abelian category whose objects are $\mathbf{C}$-vector spaces $V$ with a given vector space decomposition $\left.V=\bigoplus_{\lambda \in \mathbf{C}}{ }_{\lambda} V\right)$ and with a given $\widetilde{\mathbf{g}}$-module (resp. $\widetilde{\mathbf{g}}^{+}$-module, $\mathbf{g} \oplus \mathbf{1 C}$-module) structure such that such that:

(a) $\epsilon^{n} c\left({ }_{\lambda} V\right) \subset{ }_{\lambda-n} V$ for all $c \in \mathbf{g}$ and all $n \in \mathbf{Z}$ (resp. $n \in \mathbf{N}, n=0$ ),

(b) 1 acts as multiplication by $\kappa-h$,

(c) the action of $\mathbf{g}$ on each ${ }_{\lambda} V$ is locally finite.

We have a natural imbedding $\mathscr{O}_{\kappa} \subset \mathscr{C}$ as a full subcategory, defined by attaching to a $\tilde{\mathbf{g}}$-module $V$ in $\mathscr{O}_{\kappa}$ the direct sum decomposition defined by the generalized eigenspaces of $L_{0}: V \rightarrow V$. The following holds.

(d) For any $a, a^{\prime} \in \mathbf{N}^{I}$ and any $s \geq 1$ we have $\operatorname{Ext}_{\mathscr{C}}^{s}\left(\mathbf{V}_{a}^{\kappa}, D\left(\mathbf{V}_{a^{\prime}}^{\kappa}\right)\right)=0$.

We will sketch a proof following Polo [P].

The objects of $\mathscr{C}^{0}$ are both projective and injective. Using objects induced or "co-induced" from $\mathscr{C}^{0}$ to $\mathscr{C}, \mathscr{C}^{+}$, we see that $\mathscr{C}, \mathscr{C}^{+}$have enough projective and injective objects. We can regard $\mathscr{V}_{a}$ as an object of $\mathscr{C}^{+}$so that the corresponding induced object in $\mathscr{C}$ is $\mathbf{V}_{a}^{\kappa}$. Since induction from $\mathscr{C}^{+}$to $\mathscr{C}$ is exact and takes projectives to projectives, we see that

$$
\operatorname{Ext}_{\mathscr{C}}^{s}\left(\mathbf{V}_{a}^{\kappa}, D\left(\mathbf{V}_{a^{\prime}}^{\kappa}\right)\right)=\operatorname{Ext}_{\mathscr{C}^{+}}^{s}\left(\mathscr{V}_{a}, D\left(\mathbf{V}_{a^{\prime}}^{\kappa}\right)\right)
$$


Next one verifies that $\mathscr{V}_{\bar{a}^{\prime}}$ can be regarded as an object of $\mathscr{C}^{0}$ so that the corresponding "co-induced" object in $\mathscr{C}^{+}$is the restriction of $D\left(\mathbf{V}_{a^{\prime}}^{\kappa}\right)$. It follows that $D\left(\mathbf{V}_{a^{\prime}}^{\kappa}\right)$ is an injective object of $\mathscr{C}^{+}$. Hence, the right-hand side of (e) is zero and (d) follows.

3.8. From 3.3(a),(b), 3.4, 3.5, 3,6, 3.7(a), 2.24 we see that Soergel's axioms [So] for his abstract Brauer (or BGG) reciprocity are satisfied for $\mathscr{O}_{\kappa}^{t}$. Thus we can apply Soergel's result in our case and obtain the following result.

Proposition 3.9. For each $a \in F^{t}$ there is a projective object $P_{a}$ of $\mathscr{O}_{\kappa}^{t}$ such that:

(a) $\operatorname{dim} \operatorname{Hom}_{\mathscr{\theta}_{\kappa}}\left(P_{a}, \mathbf{L}_{a^{\prime}}^{\kappa}\right)=\delta_{a a^{\prime}}$ for $a, a^{\prime} \in F^{t}$, and

(b) $P_{a}$ admits a finite filtration with subquotients of form $\mathbf{V}_{b}^{\kappa} \quad\left(b \in F^{t}\right)$ and the number of occurences of $\mathbf{V}_{b}^{\kappa}$ is equal to $\left[\mathbf{V}_{b}^{\kappa}: \mathbf{L}_{a}^{\kappa}\right]$.

Here we have used the following notation: if $V$ is in $\mathscr{O}_{\kappa}$, we denote by $\left[V: \mathbf{L}_{a}^{\kappa}\right.$ ] the number of subquotients in a composition series of $V$ which are isomorphic to $\mathbf{L}_{a}^{\kappa}$.

To state the following result we need some further notation. For a finitedimensional g-module $\mathscr{V}$ we denote by $\left[\mathscr{V}: \mathscr{V}_{a}\right]_{\mathbf{g}}$ the number of times $\mathscr{V}_{a}$ appears in a decomposition of $\mathscr{V}$ as a direct sum of simple g-modules.

Corollary 3.10. For any $V$ in $\mathscr{O}_{\kappa}$ and any $a \in \mathbf{N}^{I}$, we have

$$
\left[V: \mathbf{L}_{a}^{\kappa}\right] \leq \sum_{b}\left[\mathbf{V}_{b}^{\kappa}: \mathbf{L}_{a}^{\kappa}\right]\left[V(1): \mathscr{V}_{b}\right]_{\mathbf{g}}
$$

(Here $b$ runs over $\mathbf{N}^{I}$; but only finitely many terms in the right-hand side are nonzero since $\operatorname{dim} V(1)<\infty$.)

We choose $t$ such that $V$ is in $\mathscr{O}_{\kappa}^{t}$.

Since $P_{a}$ is projective in $\mathscr{O}_{\kappa}^{t}$, the number $\operatorname{dim}_{\operatorname{Hom}_{\mathscr{O}_{\kappa}}}\left(P_{a}, V^{\prime}\right)$ is additive with respect to short exact sequences in $V^{\prime} \in \mathscr{O}_{\kappa}^{t}$. It follows that

$$
\begin{aligned}
\operatorname{dim} \operatorname{Hom}_{\mathscr{O}_{\kappa}}\left(P_{a}, V\right) & =\sum_{a^{\prime}} \operatorname{dim} \operatorname{Hom}_{\mathscr{O}_{\kappa}}\left(P_{a}, \mathbf{L}_{a^{\prime}}^{\kappa}\left[V: \mathbf{L}_{a^{\prime}}^{\kappa}\right]\right. \\
& =\sum_{a^{\prime}} \delta_{a a^{\prime}}\left[V: \mathbf{L}_{a^{\prime}}^{\kappa}\right]=\left[V: \mathbf{L}_{a}^{\kappa}\right] .
\end{aligned}
$$

Moreover, if $0 \rightarrow X^{\prime} \rightarrow X \rightarrow X^{\prime \prime} \rightarrow 0$ is a short exact sequence in $\mathscr{O}_{\kappa}^{t}$, then we have

$$
\operatorname{dim} \operatorname{Hom}_{\mathscr{O}_{\kappa}}(X, V) \leq \operatorname{dim} \operatorname{Hom}_{\mathscr{O}_{\kappa}}\left(X^{\prime}, V\right)+\operatorname{dim} \operatorname{Hom}_{\mathscr{O}_{\kappa}}\left(X^{\prime \prime}, V\right) .
$$

Applying this repeatedly to a filtration of $P_{a}$ as in 3.9(b), we obtain

$$
\begin{aligned}
\operatorname{dim} \operatorname{Hom}_{\mathscr{O}_{\kappa}}\left(P_{a}, V\right) & \leq \sum_{b}\left[\mathbf{V}_{b}^{\kappa}: \mathbf{L}_{a}^{\kappa}\right] \operatorname{dim} \operatorname{Hom}_{\mathscr{O}_{\kappa}}\left(\mathbf{V}_{b}^{\kappa}, V\right) \\
& =\sum_{b}\left[\mathbf{V}_{b}^{\kappa}: \mathbf{L}_{a}^{\kappa}\right] \operatorname{dim} \operatorname{Hom}_{\mathbf{g}}\left(\mathscr{V}_{b}, V(1)\right),
\end{aligned}
$$

and the corollary follows. 
3.11. Proof of Theorem 3.2. Let $V$ be a $\widetilde{\mathbf{g}}$-module as in 3.2(b). For any $N \geq$ $1, V(N)$ is finite dimensional (see $1.10(\mathrm{e})$ ) and the $\widetilde{\mathbf{g}}$-module $V\{N\}$ of $\bar{V}$ generated by $V(N)$ satisfies $2.22(\mathrm{c})$; hence, it is in $\mathscr{O}_{\kappa}$. Therefore, 3.10 may be applied to it. Since $V\{N\}(1)=V(1)$, we see from 3.10 that

$$
\text { length } V\{N\} \leq \sum_{a, b}\left[V: \mathbf{L}_{a}^{\kappa}\right] \leq \sum_{a, b}\left[\mathbf{V}_{b}^{\kappa}: \mathbf{L}_{a}^{\kappa}\right]\left[V(1): \mathscr{V}_{b}\right]_{\mathbf{g}} \text {. }
$$

The last expression is finite and independent of $N$.

It follows that the ascending sequence of $\widetilde{\mathbf{g}}$-submodules $V\{1\} \subset V\{2\} \subset \cdots$ must be stationary. The union of the terms of this sequence is equal to $V$ (since already the union of the $V(N)$ is equal to $V$ ). It follows that we must have $V\{N\}=V$ for some $N \geq 1$. Since $V\{N\}$ is in $\mathscr{O}_{\kappa}$, the same must hold for $V$.

Conversely, any $\widetilde{\mathbf{g}}$-module in $\mathscr{O}_{\kappa}$ is as in $3.2(\mathrm{~b})$, by 2.17 . The theorem is proved.

\section{FIRST DEFINITION OF TENSOR PRODUCT}

4.1. Assume that we are given $n$ smooth $\widetilde{\mathbf{g}}$-modules with the same central charge $\kappa-h$. The usual tensor product of these modules is a $\widetilde{\mathbf{g}}$-module with central charge $n(\kappa-h)$; moreover, it does not have reasonable finiteness properties when the factors do.

Our present aim is to give a modified construction of a tensor product which leads to a module with the same central charge $\kappa-h$.

Unlike the usual tensor product, the modified tensor product is defined in terms of a choice, namely, of local parameters (up to second order) at $(n+1)$ distinct points of a curve.

4.2. The setup for our definition is the following one. We assume given a finite nonempty set $S$ with a given nonempty subset $\nabla$. We assume that for each $s_{0} \in \varnothing$ we are given a subset $\left[s_{0}\right]$ of $S$ containing $s_{0}$ such that, when $s_{0}$ runs through $\odot$, the subsets $\left[s_{0}\right]$ form a partition of $S$. Thus any $s \in S$ belongs to $\left[s_{0}\right]$ for a unique $s_{0} \in \varnothing$. Let $\boldsymbol{\phi}=S-\varnothing$. We assume that each subset $\left[s_{0}\right]$ contains at least two elements (thus it contains, besides $s_{0}$, at least one element in $\boldsymbol{\$})$.

We assume given a smooth projective curve $C$ over $\mathbf{C}$, such that any connected component of $C$ is isomorphic to $P^{1}$ and such that the set of connected components of $C$ is in a given bijection $\left(s_{0} \leftrightarrow C_{s_{0}}\right)$ with the set $\odot$.

We assume that $C$ has specified distinct points $p_{s} \quad(s \in S)$ such that $p_{s} \in$ $C_{s_{0}}$ for all $s \in\left[s_{0}\right]$.

We also assume that for each $s \in S$ we are given a morphism $\gamma_{s}: P^{1} \rightarrow C$ such that $\gamma_{s}$ defines an isomorphism $P^{1} \cong C_{s_{0}}$ (where $s \in\left[s_{0}\right]$ ) and such that $\gamma_{s}(0)=p_{s}$. (These morphisms are called charts.)

4.3. Let $V_{s} \quad(s \in \mathbf{A})$ be a collection of smooth $\widetilde{\mathbf{g}}$-modules with the same central charge $\kappa-h$, indexed by the set $\boldsymbol{\phi}$. 
Our definition of tensor product will attach to the $\widetilde{\mathbf{g}}$-modules $V_{s}$ and to the system of charts $\left(\gamma_{s}\right)$, a $\widetilde{\mathbf{g}}^{\circ}$-module $T(W)^{\sharp}$.

The most important case for applications is that where $C$ is connected (so $\varnothing$ has a single element) and consists of two elements; thus, we are given two g-modules $V_{s}$.

However, for technical reasons, it is necessary to consider the more general setup described above.

4.4. An outline of the definition of $T(W)^{\sharp}$ is as follows. We will define a natural filtration of the usual tensor product of $\mathrm{C}$-vector spaces $W=\bigotimes_{s \in \boldsymbol{A}} V_{s}$, and we will form the completion $\widehat{W}$ of $W$ with respect to this filtration. We will show that this completion is naturally a $\widehat{\mathbf{g}}^{\odot}$-module. We restrict this to a $\widetilde{\mathbf{g}}^{\odot}$-module, apply to it ${ }^{\sharp}$, and in the resulting $\widetilde{\mathbf{g}}^{\odot}$-module we take the subspace of smooth vectors. This subspace will be $T(W)^{\sharp}$.

4.5. Let $C^{\prime}$ be the affine open curve obtained from $C$ by removing the points $p_{s}(s \in S)$. Let $R$ be the algebra of all regular functions $C^{\prime} \rightarrow \mathbf{C}$.

For any $s \in S$, we have an algebra homomorphism

$$
R \rightarrow \mathbf{C}((\epsilon)) \quad\left(f \mapsto{ }^{s} f\right)
$$

defined as follows:

$$
\begin{aligned}
& { }^{s} f \text { is the power series expansion at } 0 \text { of the rational function } \\
& f \gamma_{s} \text { on } P^{1} \text {. }
\end{aligned}
$$

4.6. The Lie algebra $\Gamma$. For $f_{1}, f_{2}$ in $R$, let $\left\{f_{1}, f_{2}\right\}$ be the sum of residues of the differential form $f_{2} d\left(f_{1}\right)$ on $C^{\prime}$ at the points $p_{s}(s \in \nabla)$. The bilinear pairing $\{\}:, R \times R \rightarrow \mathbf{C}$ satisfies

$$
\left\{f_{1}, f_{2}\right\}+\left\{f_{2}, f_{1}\right\}=0, \quad\left\{f_{1} f_{2}, f_{3}\right\}+\left\{f_{2} f_{3}, f_{1}\right\}+\left\{f_{3} f_{1}, f_{2}\right\}=0
$$

for all $f_{1}, f_{2}, f_{3} \in R$.

Hence, $\Gamma=(R \otimes \mathbf{g}) \oplus \mathbf{C 1}$ has a natural structure of $\mathbf{C}$-Lie algebra with $\mathbf{1}$ in the centre and with $\left[f c, f^{\prime} c^{\prime}\right]=f f^{\prime}\left[c, c^{\prime}\right]+\left\{f, f^{\prime}\right\}\left(c, c^{\prime}\right) \mathbf{1}$ for all $f, f^{\prime} \in R$ and all $c, c^{\prime} \in \mathbf{g}$. (We write $f c$ instead of $f \otimes c$.)

We have natural Lie algebra homomorphisms

$$
\begin{gathered}
\Gamma \rightarrow \widehat{\mathbf{g}}^{\odot}, \\
\Gamma \rightarrow \widehat{\mathbf{g}}^{\bullet} .
\end{gathered}
$$

The map (a) is given by

$$
f c \mapsto \sum_{s \in \mathcal{O}} \delta_{s}^{s} f c \quad \text { and } \quad \mathbf{1} \mapsto \mathbf{1}
$$

the map (b) is given by

$$
f c \mapsto \sum_{s \in \boldsymbol{A}} \delta_{s}^{s} f c \quad \text { and } \quad \mathbf{1} \mapsto-\mathbf{1} .
$$

The fact that (a) is a Lie algebra homomorphism is obvious. The fact that (b) is a Lie algebra homomorphism is a consequence of the following fact: if 
$f_{1}, f_{2}$ are functions in $R$, then the sum of the residues at $p_{s}(s \in \boldsymbol{\$})$ of the differential form $f_{2} d\left(f_{1}\right)$ on $C^{\prime}$ is equal to minus the sum of residues of that form at $p_{s} \quad(s \in \mathcal{O})$. (This follows from the residue theorem since that differential form is regular on $C^{\prime}$.)

4.7. $\Gamma$-module structure on $W$. Now $W$ is naturally a $\widehat{\mathbf{g}}^{\text {m-module since the }}$ $V_{s}$ have the same central charge. (See 1.13.) By restriction, $W$ is therefore also a $\Gamma$-module. Note that $1 \in \Gamma$ acts on $W$ as $(-\kappa+h)$ times the identity.

4.8. Definition of a decreasing filtration of $W$. For any $n \in \mathbf{Z}$, let $R_{n}$ be the subspace of $R$ consisting of those $f$ such that $\left.s_{0} f \in \epsilon^{n} \mathbf{C}[[\epsilon]]\right\}$ for all $s_{0} \in \nabla$. We have $\cdots \subset R_{n} \subset R_{n-1} \subset \cdots$. Not that $R_{n} R_{m} \subset R_{n+m}$ for all $n, m$.

For any integer $N \geq 1$, let $G_{N}$ be the C-subspace of $U(\Gamma)$ spanned by all products $\left(f_{1} c_{1}\right)\left(f_{2} c_{2}\right) \cdots\left(f_{N} c_{N}\right)$ with $f_{1}, f_{2}, \ldots, f_{N} \in R_{1}$ and $c_{1}, c_{2}, \ldots$, $c_{N} \in \mathbf{g}$. It is convenient to define $G_{0}$ as the set of all $\mathbf{C}$-multiples of the unit element in $U(\Gamma)$.

The vector space $W$ has the following natural decreasing filtration

$$
W \supset G_{1} W \supset G_{2} W \supset \cdots
$$

where $G_{N} W$ is defined using the $U(\Gamma)$-module structure on $W$.

This gives rise to a projective system of vector spaces:

$$
W / G_{1} W \leftarrow W / G_{2} W \leftarrow W / G_{3} W \leftarrow \cdots ;
$$

the corresponding projective limit is denoted $\widehat{W}$.

4.9. $\widehat{\mathbf{g}}$-module structure on $\widehat{W}$. We state the following three facts $((\mathrm{a})$ is left to the reader and (b),(c) will be verified in 4.12, 4.13):

(a) (Approximation property.) Assume that for'each $s_{0} \in \varnothing$ we are given a power series $\omega_{s_{0}} \in \mathbf{C}((\epsilon))$. Then, for any $N \geq 0$, there exists $g \in R$ such that ${ }^{s_{0}} g-\omega_{s_{0}} \in \epsilon^{N} \mathbf{C}[[\epsilon]]$ for all $s_{0} \in \mathcal{O}$.

(b) Let $N \geq 1$ and let $g \in R_{N}, c \in \mathbf{g}$. Then $g c \in G_{N}$.

(c) Let $t \geq r \geq 0$. Let $c \in \mathbf{g}$ and $f \in R_{-r}$. We have $(f c) G_{t} \subset G_{t-r} U(\Gamma)$.

Let $\omega=\left(\omega_{s}\right)_{s \in \odot} \in \mathbf{C}((\epsilon))^{\odot}$ and let $c \in \mathbf{g}$.

We define a C-linear map $\langle\omega c\rangle: \widehat{W} \rightarrow \widehat{W}$ as follows. We choose a sequence $g_{1}, g_{2}, \ldots$ of elements of $R$ such that ${ }^{s} g_{n}-\omega_{s} \in \epsilon^{n} \mathbf{C}[[\epsilon]]$ for $n=1,2, \ldots$ and all $s \in \mathcal{O}$. (See (a).) We also choose $q \geq 0$ such that $\omega_{s} \in \epsilon^{-q} \mathbf{C}[[\epsilon]]$ for all $s \in \mathcal{O}$. We represent an element of $\widehat{W}$ by a sequence $\left(x_{1}, x_{2}, \ldots\right)$ of elements in $W$ such that $x_{n+1}-x_{n} \in G_{n} W$ for $n=1,2, \ldots$

We have ${ }^{s}\left(g_{n+1}-g_{n}\right) \in \epsilon^{n} \mathbf{C}[[\epsilon]]$ for all $s \in \odot$; hence, by (b),

$$
\left(g_{n+1}-g_{n}\right) c \in G_{n} \text {. }
$$

We have $g_{n+1} \in R_{-q}$ by the choice of $q$; hence, by (c),

$$
\left(g_{n+1} c\right) G_{q+n} W \subset G_{n} W .
$$

We set

$$
\langle\omega c\rangle\left(x_{1}, x_{2}, \ldots\right)=\left(\left(g_{1} c\right) x_{q+1},\left(g_{2} c\right) x_{q+2}, \ldots\right)
$$


(Here, $\left(g_{n} c\right) x_{q+n}$ is defined by the $\Gamma$-module structure of $W$.) The right-hand side of (f) defines an element of $\widehat{W}$; indeed,

$$
\begin{aligned}
& \left(g_{n+1} c\right) x_{q+n+1}-\left(g_{n} c\right) x_{q+n} \\
& \quad=\left(g_{n+1} c\right)\left(x_{q+n+1}-x_{q+n}\right)+\left(\left(g_{n+1}-g_{n}\right) c\right) x_{q+n} \\
& \quad \in\left(g_{n+1} c\right) G_{q+n} W+G_{n} W \subset G_{n} W+G_{n} W \subset G_{n} W .
\end{aligned}
$$

(We have used (d), (e).)

We show that the map $\langle\omega c\rangle$ is well defined. Let $g_{1}^{\prime}, g_{2}^{\prime}, \ldots$ be another sequence of elements in $R$ such that ${ }^{s} g_{n}^{\prime}-\omega_{s} \in \epsilon^{n} \mathbf{C}[[\epsilon]]$ for $n=1,2, \ldots$ and for all $s \in \odot$; let $q^{\prime} \geq 0$ be such that $\omega_{s} \in \epsilon^{-q^{\prime}} \mathbf{C}[[\epsilon]]$ for all $s \in \odot$.

We assume that $q^{\prime} \geq q$.

Let $\left(x_{1}^{\prime}, x_{2}^{\prime}, \ldots\right)$ be a sequence of elements in $W$ such that $x_{n}^{\prime}-x_{n} \in G_{n} W$ for all $n \geq 1$.

We have $g_{n}^{\prime}-g_{n} \in R_{n}$; hence, by (b),

$$
\left(g_{n}^{\prime}-g_{n}\right) c \in G_{n} \text {. }
$$

We have $g_{n} \in R_{-q}$ by the choice of $q$ and $x_{q^{\prime}+n}-x_{q+n} \in G_{q+n} W$ (by the definition of $\left(x_{n}\right)$ and by the assumption $\left.q^{\prime} \geq q\right)$; hence, by (c),

$$
\left(g_{n} c\right)\left(x_{q^{\prime}+n}-x_{q+n}\right) \in G_{n} W .
$$
by (c),

We have $g_{n}^{\prime} \in R_{-q^{\prime}}$ by the choice of $q^{\prime}$ and $x_{q^{\prime}+n}^{\prime}-x_{q^{\prime}+n} \in G_{q^{\prime}+n} W$; hence,

$$
\left(g_{n}^{\prime} c\right)\left(x_{q^{\prime}+n}^{\prime}-x_{q^{\prime}+n}\right) \in G_{n} W
$$

Using (g), (h), (i), we see that

$$
\begin{aligned}
\left(g_{n}^{\prime} c\right) x_{q^{\prime}+n}^{\prime}-\left(g_{n} c\right) x_{q+n}= & \left(\left(g_{n}^{\prime}-g_{n}\right) c\right) x_{q^{\prime}+n} \\
& +\left(g_{n}^{\prime} c\right)\left(x_{q^{\prime}+n}^{\prime}-x_{q^{\prime}+n}\right)+\left(g_{n} c\right)\left(x_{q^{\prime}+n}-x_{q+n}\right) \in G_{n} W .
\end{aligned}
$$

In other words, we have

$$
\left(\left(g_{1} c\right) x_{q+1},\left(g_{2} c\right) x_{q+2}, \ldots\right)=\left(\left(g_{1}^{\prime} c\right) x_{q^{\prime}+1}^{\prime},\left(g_{2}^{\prime} c\right) x_{q^{\prime}+2}^{\prime}, \ldots\right)
$$

as elements of $\widehat{W}$. This shows that the map $\langle\omega c\rangle$ is well defined.

Lemma 4.10. The endomorphisms $\langle\omega c\rangle$ define a $\widehat{\mathbf{g}}^{\circ}$-module structure on $\widehat{W}$ with 1 acting as multiplication by $-\kappa+h$.

Let $\widetilde{\omega}=\left(\widetilde{\omega}_{s}\right)_{s \in \mathcal{O}} \in \mathbf{C}((\epsilon))^{\odot}$ and let $\widetilde{c} \in \mathbf{g}$.

We choose a sequence $\widetilde{g}_{1}, \widetilde{g}_{2}, \ldots$ of elements of $R$ such that ${ }^{s} \widetilde{g}_{n}-\widetilde{\omega}_{s} \in$ $\epsilon^{n} \mathrm{C}[[\epsilon]]$ for $n=1,2, \ldots$ and for all $s \in \mathcal{O}$. (See 4.9(a).) We also choose $t \geq 0$ such that $\widetilde{\omega}_{s} \in \epsilon^{-t} \mathbf{C}[[\epsilon]]$ for all $s \in \mathcal{O}$.

Let $\omega \widetilde{\omega}=\left(\omega_{s} \widetilde{\omega}_{s}\right)_{s \in \mathcal{O}} \in \mathbf{C}((\epsilon))^{\odot} ;$ then $\langle\omega \tilde{\omega}[c, \widetilde{c}]\rangle: \widehat{W} \rightarrow \widehat{W}$ is well defined.

Let $h_{n}=g_{n+t} \widetilde{g}_{n+q}$. Then $\left.{ }^{s} h_{n}-\omega_{s} \widetilde{\omega}_{s} \in \epsilon^{n} \mathbf{C}[\epsilon]\right]$ for $n=1,2, \ldots$ and for all $s \in \mathcal{O}$. 
From the definitions, the commutator of the endomorphisms $\langle\omega c\rangle$ and $\left\langle\omega^{\prime} c^{\prime}\right\rangle$ applied to $\left(x_{1}, x_{2}, \ldots\right)$ gives the element of $\widehat{W}$ represented by $\left(y_{1}\right.$, $\left.y_{2}, \ldots\right)$ where

$$
\begin{aligned}
y_{n}= & \left(g_{n} c\right)\left(\widetilde{g}_{n+q} \widetilde{c}\right) x_{q+t+n}-\left(\widetilde{g}_{n} \widetilde{c}\right)\left(g_{n+t} c\right) x_{q+t+n} \\
= & \left(\widetilde{g}_{n+q} \widetilde{c}\right)\left(g_{n} c\right) x_{q+t+n}-\left(\widetilde{g}_{n} \widetilde{c}\right)\left(g_{n+t} c\right) x_{q+t+n} \\
& +\left(g_{n} \widetilde{g}_{n+q}[c, \widetilde{c}] x_{q+t+n}+(-\kappa+h)\left\{g_{n}, \widetilde{g}_{n+q}\right\}(c, \widetilde{c}) x_{q+t+n} .\right.
\end{aligned}
$$

On the other hand, the endomorphism

$$
\langle\omega \widetilde{\omega}[c, \widetilde{c}]\rangle+(-\kappa+h) \sum_{s \in \mathcal{S}}\left\{\omega_{s}, \widetilde{\omega}_{s}\right\}(c, \widetilde{c}) \operatorname{Id}_{\widehat{W}}
$$

applied to $\left(x_{1}, x_{2}, \ldots\right)$ gives the element of $\widehat{W}$ represented by $\left(z_{1}, z_{2}, \ldots\right)$ where

$$
z_{n}=\left(h_{n}[c, \widetilde{c}]\right) x_{q+t+n}+(-\kappa+h) \sum_{s \in \mathcal{O}}\left\{\omega_{s}, \widetilde{\omega}_{s}\right\}(c, \widetilde{c}) x_{n} .
$$

We must prove that $\left(y_{1}, y_{2}, \ldots\right)$ and $\left(z_{1}, z_{2}, \ldots\right)$ represent the same element of $\widehat{W}$.

It is enough to show that $y_{n}-z_{n} \in G_{n-t} W$ for all $n>t$. (Then for all $n \geq 1$ we have $y_{n+t}-z_{n+t} \in G_{n} W$, and, since $y_{n+t}-y_{n} \in G_{n} W, z_{n+t}-z_{n} \in G_{n} W$, it would follow that $y_{n}-z_{n} \in G_{n} W$ for all $n$.)

It is therefore enough to show that for all $n>t$ we have

$$
\begin{gathered}
\left(\widetilde{g}_{n+q} \widetilde{c}\right)\left(g_{n} c\right) x_{q+t+n}-\left(\widetilde{g}_{n} \widetilde{c}\right)\left(g_{n+t} c\right) x_{q+t+n} \in G_{n-t} W, \\
\left(\left(g_{n} \widetilde{g}_{n+q}-g_{n+t} \widetilde{g}_{n+q}\right)[c, \widetilde{c}]\right) x_{q+t+n} \in G_{n-t} W,
\end{gathered}
$$

and

$$
\left\{g_{n}, \widetilde{g}_{n+q}\right\}(c, \widetilde{c}) x_{q+t+n}-\sum_{s \in \mathcal{S}}\left\{\omega_{s}, \widetilde{\omega}_{s}\right\}(c, \widetilde{c}) x_{n} \in G_{n-t} W .
$$

We have $\omega_{s}={ }^{s} g_{n}+\epsilon^{n} r_{s}, \quad \widetilde{\omega}_{s}={ }^{s} \widetilde{g}_{n+q}+\epsilon^{n+q} r_{s}^{\prime}$ with $r, r^{\prime} \in \mathbf{C}[[\epsilon]]$. Thus,

$$
\begin{aligned}
\left\{g_{n}, \widetilde{g}_{n+q}\right\} & =\sum_{s \in \mathcal{S}}\left\{\omega_{s}-\epsilon^{n} r_{s}, \widetilde{\omega}_{s}-\epsilon^{n+q} r_{s}^{\prime}\right\} \\
& =\sum_{s \in \mathcal{S}}\left(\left\{\omega_{s}, \widetilde{\omega}_{s}\right\}-\left\{\omega_{s}, \epsilon^{n+q} r_{s}^{\prime}\right\}-\left\{\epsilon^{n} r_{s}, \widetilde{\omega}_{s}\right\}+\left\{\epsilon^{n} r_{s}, \epsilon^{n+q} r_{s}^{\prime}\right\}\right) .
\end{aligned}
$$

Since $\epsilon^{q} \omega_{s}, \epsilon^{n} \widetilde{\omega}_{s}, \epsilon^{n} r_{s}^{\prime}, r_{s}$ are in $\left.\mathbf{C}[\epsilon]\right]$ (recall that $n>t$ ), we see that the last three residues are zero; hence, $\left\{g_{n}, \widetilde{g}_{n+q}\right\}=\sum_{s \in \mathcal{O}}\left\{\omega_{s}, \widetilde{\omega}_{s}\right\}$. This together with $x_{q+t+n}-x_{n} \in G_{n} W$ implies (c).

We have $g_{n}-g_{n+t} \in R_{n}$ and $\widetilde{g}_{n+q} \in R_{-t}$; hence, $\left(g_{n}-g_{n+t}\right) \widetilde{g}_{n+q} \in R_{n-t}$ so that (b) follows from 4.9(b) (recall that $n>t$ ).

The expression in (a) is equal to the sum of four terms:

$$
\begin{array}{cc}
\left(\left(\widetilde{g}_{n+q}-\widetilde{g}_{n}\right) \widetilde{c}\right)\left(g_{n} c\right) x_{q+t+n}, & \left(\left(g_{n}-g_{n+t}\right) c\right)\left(\widetilde{g}_{n} \widetilde{c}\right) x_{q+t+n}, \\
\left(\widetilde{g}_{n}\left(g_{n}-g_{n+t}\right)[\tilde{c}, c]\right) x_{q+t+n}, & (-\kappa+h)\left\{\widetilde{g}_{n}, g_{n}-g_{n+t}\right\}(\widetilde{c}, c) x_{q+t+n} .
\end{array}
$$


It is enough to show that each of these four terms is in $G_{n-t} W$. We have $\widetilde{g}_{n+q}-\widetilde{g}_{n} \in R_{n}$; hence, by $4.9(\mathrm{~b})$, the first term is in $G_{n} W$ hence in $G_{n-t} W$.

Similarly, we have $g_{n}-g_{n+t} \in R_{n}$; hence, by $4.9(\mathrm{~b})$, the second term is in $G_{n} W$ and, hence, in $G_{n-t} W$.

This shows also that $\widetilde{g}_{n}\left(g_{n}-g_{n+t}\right) \in R_{n-t}$ (recall that $\left.\widetilde{g}_{n} \in R_{-t}\right)$. Using again $4.9(\mathrm{~b})$, we see that the third term is in $G_{n-t} W$.

To deal with the fourth term it is enough to show that $\left\{\widetilde{g}_{n}, g_{n}-g_{n+t}\right\}=0$. This follows from $\widetilde{g}_{n} \in R_{-t}, g_{n}-g_{n+t} \in R_{n}$ and from $n>t$.

This completes the proof of the lemma.

4.11. The definition of $T(W)^{\sharp}$. We restrict the $\widehat{\mathbf{g}}^{\mathcal{O}}$-module $\widehat{W}$ to $\widetilde{\mathbf{g}}^{\circ}$ using the obvious imbedding $\widetilde{\mathbf{g}}^{\odot} \subset \widehat{\mathbf{g}}^{\odot}$. We now consider the $\widetilde{\mathbf{g}}^{\odot}$-submodule $T(W)=$ $\widehat{W}(-\infty)$ of the $\widetilde{\mathbf{g}}^{\bigcirc}$-module $\widehat{W}$ (see 1.9). Hence the $\widetilde{\mathbf{g}}^{\bigcirc}$-module $T(W)^{\sharp}$ is defined (see 1.5). It has central charge $\kappa-h$.

We get the same $\widetilde{\mathbf{g}}^{\odot}$-module if we first apply ${ }^{\sharp}$ to the $\widetilde{\mathbf{g}}^{\odot}$-module $\widehat{W}$ and then take the set of smooth vectors: $\left(\widehat{W}^{\sharp}\right)(\infty)$.

4.12. Proof of 4.9(b). We argue by induction on $N$. The case where $N=1$ is trivial. Now assume that $N>1$. If $g \in R_{N}$, we can write $g$ as a sum of products $g^{\prime} g^{\prime \prime}$ where $g^{\prime} \in R_{N^{\prime}}, g^{\prime \prime} \in R_{N^{\prime \prime}}$ and $N^{\prime}+N^{\prime \prime}=N, N^{\prime} \geq 1, N^{\prime \prime} \geq 1$. Also we can write $c$ as a sum of commutators $\left[c^{\prime}, c^{\prime \prime}\right]$ in $\mathbf{g}$. Clearly, we may assume that both sums have a single term: $g=g^{\prime} g^{\prime \prime}$ and $c=\left[c^{\prime}, c^{\prime \prime}\right]$. We have $g c=\left[g^{\prime} c^{\prime}, g^{\prime \prime} c^{\prime \prime}\right]$ in $\Gamma$; hence, $g c=\left(g^{\prime} c^{\prime}\right)\left(g^{\prime \prime} c^{\prime \prime}\right)-\left(g^{\prime \prime} c^{\prime \prime}\right)\left(g^{\prime} c^{\prime}\right)$ in $U(\Gamma)$.

By the induction hypothesis, this is contained in $G_{N^{\prime}} G_{N^{\prime \prime}}+G_{N^{\prime \prime}} G_{N^{\prime}} \subset G_{N}$ as required.

4.13. Proof of 4.9(c). We argue by induction on $t$. The case where $t=r$ is trivial. We now assume that $t>r$. Let $f_{1}, f_{2}, \ldots, f_{t}$ be elements of $R_{1}$ and let $c_{1}, c_{2}, \ldots, c_{t}$ be elements of $\mathbf{g}$. Let $\xi=\left(f_{2} c_{2}\right) \cdots\left(f_{t} c_{t}\right)$.

The product $(f c)\left(f_{1} c_{1}\right) \xi$ is equal to the sum of three terms:

$$
\left(f_{1} c_{1}\right)(f c) \xi+\left(f f_{1}\left[c, c_{1}\right]\right) \xi+\left\{f, f_{1}\right\}\left(c, c_{1}\right) \mathbf{1} \xi .
$$

The first term is in $G_{1} G_{t-r-1} U(\Gamma)$ (by the induction hypothesis). The second term is in $G_{t-r} U(\Gamma)$ (trivially, if $r=0$, and by the induction hypothesis, if $r \geq 1$ ). The third term is in $G_{t-r} U(\Gamma)$ (trivially, if $r \geq 1$, and since $\left\{f, f_{1}\right\}=0$, if $\left.r=0\right)$. This completes the proof.

4.14. Remarks. For any $s \in S$ with $s \in\left[s_{0}\right] \quad\left(s_{0} \in \varnothing\right)$, let $f_{s} \in R$ be the function defined by

$$
f_{s}(p)=\frac{1}{\gamma_{s}^{-1}(p)} \text { for } p \in C_{s_{0}} \text { and } f_{s}=0 \text { on } C-C_{s_{0}} .
$$

This function is regular on $C-\left\{p_{s}\right\}$ and satisfies ${ }^{s} f_{s}=\frac{1}{\epsilon}$.

A system of charts $\left(\gamma_{s}\right)$ on $C$ is said to be special if, for any $s \in \mathcal{\nabla}$, the point $\gamma_{s}(\infty)$ belongs to the set $\left\{p_{s^{\prime}} \mid s^{\prime} \in \boldsymbol{\phi}\right\}$.

An equivalent condition is that $f_{s}$ does not vanish on $C^{\prime}$ if $s \in \Theta$. In this case, the function $f_{s}^{-n}$ belongs to $R$ for any $n \in \mathbf{Z}$ and we have ${ }^{s}\left(f_{s}^{-n}\right)=\epsilon^{n}$; 
therefore, we have a simple formula for the action of $\delta_{s} \epsilon^{n} c \in \widetilde{\mathbf{g}}^{\circ}$ (with $c \in$ $\mathbf{g}, n \in \mathbf{Z}, s \in \mathcal{O}$ on $\widehat{W}$ (or $\widehat{W}(-\infty))$, namely,

$$
\left(\delta_{s} \epsilon^{n} c\right)\left(y_{1}, y_{2}, \ldots\right)=\left(\left(f_{s}^{-n} c\right) y_{q+1},\left(f_{s}^{-n} c\right) y_{q+2}, \ldots\right)
$$

where $q \in \mathbf{N}$ is large enough.

If $\left(\gamma_{s}\right)$ is not necessarily special, the formula (a) remains valid provided that $n \leq 0$ (we can take $q \geq-n$ ); however, for $n>0$, the action of $\delta_{s} \epsilon^{n} c$ must be defined by the more complicated procedure explained earlier, involving approximations.

4.15. Relation to coinvariants. Let $X$ be a smooth $\widetilde{\mathbf{g}}^{\bigcirc}$-module with central charge $(\kappa-h)$. Then $X$ extends naturally to a $\widehat{\mathbf{g}}^{\circ}$-module (see 1.11 ) which then can be restricted to a $\Gamma$-module using the homomorphism of Lie algebras $\Gamma \rightarrow \widehat{\mathbf{g}}^{\circ}($ see $4.6(\mathrm{a}))$.

The tensor product $W \otimes X$ of the $\Gamma$-modules $W, X$ is a $\Gamma$-module in the standard way; it is clear that 1 acts as zero on this $\Gamma$-module.

On the other hand, the tensor product $T(W) \otimes X$ of the $\widetilde{\mathbf{g}}^{\odot}$-modules $T(W)$, $X$ is a $\widetilde{\mathbf{g}}^{\bigcirc}$-module in the standard way; again it is clear that $\mathbf{1}$ acts as zero on this $\widetilde{\mathbf{g}}^{\bigcirc}$-module.

We show that

(a) if $x \in G_{t} W, v \in X(N)$, and $t \geq N$, then $x \otimes v \in G_{1}(W \otimes X)$.

(In the last expression we use the restriction of the $\Gamma$-module structure to the Lie subalgebra $G_{1}$ of $\Gamma$.)

We can assume that $x=\xi_{1} \ldots \xi_{t} w$ with $\xi_{1}, \ldots, \xi_{t} \in G_{1}$ and $w \in W$. By definition, $\xi_{1}\left(\left(\xi_{2} \cdots \xi_{t} w\right) \otimes v\right)=\left(\xi_{1} \xi_{2} \cdots \xi_{t} w\right) \otimes v+\left(\xi_{2} \cdots \xi_{t} w\right) \otimes \xi_{1} v$; hence, $\left(\xi_{1} \xi_{2} \cdots \xi_{t} w\right) \otimes v$ is equal to $-\left(\xi_{2} \cdots \xi_{t} w\right) \otimes \xi_{1} v$ modulo $G_{1}(W \otimes X)$. Similarly, $\left(\xi_{2} \xi_{3} \cdots \xi_{t} w\right) \otimes v$ is equal to $-\left(\xi_{3} \cdots \xi_{t} w\right) \otimes\left(\xi_{2} \xi_{1} v\right)$ modulo $G_{1}(W \otimes X)$.

Continuing in this fashion, we see that $\left(\xi_{1} \xi_{2} \cdots \xi_{t} w\right) \otimes v$ is equal to $\pm w \otimes\left(\xi_{t} \cdots \xi_{2} \xi_{1} v\right)$ modulo $G_{1}(W \otimes X)$. Since $t \geq N$, we have $\xi_{t} \cdots \xi_{2} \xi_{1} v=0$ by the definition of $X(N)$. Thus (a) is proved.

\subsection{We will define a canonical C-linear map}

$$
\nu: T(W) \otimes X \rightarrow(W \otimes X) / G_{1}(W \otimes X) .
$$

If $t \geq N$, the C-linear map $W \otimes X(N) \rightarrow W \otimes X$, induced by the obvious inclusion $X(N) \rightarrow X$, takes the image of $G_{t} W \otimes X(N) \rightarrow W \otimes X(N)$ into the subspace $G_{1}(W \otimes X)$ of $W \otimes X$ (see $\left.4.15(\mathrm{a})\right)$; hence, it induces by passage to quotients a C-linear map

$$
\left(W / G_{t} W\right) \otimes X(N) \rightarrow(W \otimes X) / G_{1}(W \otimes X) .
$$

We consider the C-linear map $\widehat{W} \otimes X(N) \rightarrow\left(W / G_{t} W\right) \otimes X(N)$ (the canonical map $\widehat{W} \rightarrow W / G_{t} W$ on the first factor and the identity map on the $X(N)$-factor) for some $t \geq N$; its composition with (b) is a C-linear map

$$
\widehat{W} \otimes X(N) \rightarrow(W \otimes X) / G_{1}(W \otimes X) .
$$


From the definitions it is clear that the map (c) is independent of the choice of $t$. It is also clear that if $N^{\prime} \geq N$, the maps (c) defined in terms of $N$ and $N^{\prime}$ are compatible with the linear map $\widehat{W} \otimes X(N) \rightarrow \widehat{W} \otimes X\left(N^{\prime}\right)$ induced by the canonical imbedding $X(N) \subset X\left(N^{\prime}\right)$. Hence the maps (c) define a linear $\operatorname{map} \lim _{N \geq 1}(\widehat{W} \otimes X(N)) \rightarrow(W \otimes X) / G_{1}(W \otimes X)$ or, equivalently, $\widehat{W} \otimes X \rightarrow$ $(W \otimes X) / G_{1}(W \otimes X)$. (We use our assumption that $X$ is smooth.) Composing the last linear map with the linear map $T(W) \otimes X \rightarrow \widehat{W} \otimes X$ induced by $T(W) \subset \widehat{W}$, we obtain the linear map (a).

4.17. The map $\nu$ in 4.16(a) has the following property:

$$
\nu\left(\widetilde{\mathbf{g}}^{\odot}(T(W) \otimes X)\right) \text { is contained in } \Gamma(W \otimes X) / G_{1}(W \otimes X) .
$$

Indeed, let $\tau \in T(W), \sigma \in X, s \in \mathcal{O}, a \in \mathbf{Z}$ and $c \in \mathbf{g}$. We must show that $\left.\nu\left(\delta_{s} \epsilon^{a} c\right)(\tau \otimes \sigma)\right) \in \Gamma(W \otimes X) / G_{1}(W \otimes X)$. We represent $\tau=\left(x_{1}, x_{2}, \ldots\right)$. Assume first that $a \leq 0$. We have

$$
\left(\delta_{s} \epsilon^{a} c\right)(\tau \otimes \sigma)=\left(\left(f_{s}^{-a} c\right) x_{1-a},\left(f_{s}^{-a} c\right) x_{2-a}, \ldots\right) \otimes \sigma+\tau \otimes\left(\delta_{s} \epsilon^{a} c\right) \sigma
$$

and

$$
\nu\left(\left(\delta_{s} \epsilon^{a} c\right)(\tau \otimes \sigma)\right)=\left(f_{s}^{-a} c\right) x_{t} \otimes \sigma+x_{t} \otimes \delta_{s}\left(\epsilon^{a} c\right) \sigma=\left(f_{s}^{-a} c\right)\left(x_{t} \otimes \sigma\right)
$$

for sufficiently large $t$, and this is in the image of the $\Gamma$-action, as required.

Assume next that $a>0$. Choose a sequence $g_{1}, g_{2}, \ldots$ in $R$ such that ${ }^{s} g_{n}-\epsilon^{a} \in \epsilon^{n} \mathbf{C}[[\epsilon]]$ for all $n$ and all $s \in \mathcal{Q}$. We have

$$
\left(\delta_{s} \epsilon^{a} c\right)(\tau \otimes \sigma)=\left(\left(g_{1} c\right) x_{1},\left(g_{1} c\right) x_{2}, \ldots\right) \otimes \sigma+\tau \otimes\left(\delta_{s} \epsilon^{a} c\right) \sigma
$$

and

$$
\begin{gathered}
\nu\left(\left(\delta_{s} \epsilon^{a} c\right)(\tau \otimes \sigma)\right)=\left(g_{t} c\right) x_{t} \otimes r+x_{t} \otimes\left(\delta_{s} \epsilon^{a} c\right) \sigma \\
\nu\left(\left(\delta_{s} \epsilon^{a} c\right)(\tau \otimes \sigma)\right)=\left(g_{t} c\right)\left(x_{t} \otimes r\right)+x_{t} \otimes\left(\left(-{ }^{s} g_{t}+\epsilon^{a}\right) c\right) \sigma
\end{gathered}
$$

for sufficiently large $t$. The first term is in the image of the $\Gamma$-action; the second term is zero for large $t$, by the definition of $g_{t}$.

This proves (a).

4.18. From $4.17(a)$ we see that the linear map 4.16(a) induces a linear map

$$
(T(W) \otimes X)_{\tilde{\mathbf{g}}^{\odot}} \rightarrow(W \otimes X)_{\Gamma}
$$

\section{SECOND DEFINITION OF TENSOR PRODUCT}

The results in this section will not be used elsewhere in the paper.

5.1. We preserve the setup of 4.2 and the notation of $\S 4$. We will give an alternative definition of $T(W)$ (see 4.11) as a $\underset{\lim }{\longrightarrow} \underset{\lim }{\longleftarrow}()$.

5.2. Let $H_{1}$ be the subspace of $\Gamma$ spanned by the elements $f_{s} c \quad(s \in \odot, c \in \mathbf{g})$, where $f_{s}$ is as in 4.14 .

For any integer $r \geq 1$, let $H_{r}$ be the subspace of $U(\Gamma)$ spanned by the products $\left(f_{s_{1}} c_{1}\right)\left(f_{s_{2}} c_{2}\right) \cdots\left(f_{s_{r}} c_{r}\right)$ for various $c_{1}, c_{2}, \ldots, c_{r} \in \mathbf{g}$ and $s_{1}, s_{2}, \ldots$, $s_{r} \in Q$. 
Since $f_{s_{1}}, f_{s_{2}}, \ldots \in R_{-1}$, we have (by repeated application of $4.9(\mathrm{c})$ )

$$
H_{r} G_{t} \subset G_{t-r} U(\Gamma) \text { for all } t \geq r \geq 1 \text {. }
$$

For $t \geq r \geq 1$ we can consider the following C-subspace of $W$ :

$$
W_{t, r}=\left\{x \in W \mid \xi x \in G_{t-r} W \quad \forall \xi \in H_{r}\right\}
$$

and its quotient space

$$
\bar{W}_{t, r}=W_{t, r} / G_{t} W
$$

(We have $G_{t} W \subset W_{t, r}$ by (a).) The inclusions $W_{t, 1} \subset W_{t, 2} \subset \cdots \subset W_{t, t}$ induce by passage to quotients imbeddings

$$
\bar{W}_{t, 1} \subset \bar{W}_{t, 2} \subset \cdots \subset \bar{W}_{t, t} .
$$

The inclusions

$$
\cdots \subset W_{r+2, r} \subset W_{r+1, r} \subset W_{r, r}
$$

and

$$
\cdots \subset G_{r+2} W \subset G_{r+1} W \subset G_{r} W
$$

induce by passage to quotients $\mathbf{C}$-linear maps

$$
\cdots \rightarrow \bar{W}_{r+2, r} \rightarrow \bar{W}_{r+1, r} \rightarrow \bar{W}_{r, r} .
$$

For any integer $r \geq 1$, we consider the $\mathbf{C}$-subspace $\dot{W}_{r}$ of the product $\prod_{t \geq r} \bar{W}_{t, r}$ consisting of families of elements $x_{t, r} \in \bar{W}_{t, r}$ (for our fixed $r$ ) which are compatible with each other under the maps (c). Equivalently,

$$
\dot{W}_{r}=\underbrace{\lim }_{t} \bar{W}_{t, r} .
$$

The imbeddings (b) define natural imbeddings $\dot{W}_{1} \subset \dot{W}_{1} \subset \dot{W}_{2} \subset \ldots$. We define $\dot{W}=\bigcup_{r \geq 1} \dot{W}_{r}$. Thus, $\dot{W}=\varliminf_{r} \lim _{t} \bar{W}_{t, r}$.

5.3. We will construct a natural isomorphism of $\mathbf{C}$-vector spaces

$$
T(W)=\widehat{W}(-\infty) \cong \dot{W} .
$$

We consider an element $x \in \widehat{W}(-\infty)$. We represent it by a sequence $\left(x_{1}, x_{2}, \ldots\right)$ of elements in $W$ such that $x_{n+1}-x_{n} \in G_{n} W$ for $n=1,2, \ldots$ By the definition of $\widehat{W}(-\infty)$, there exists an $r \geq 1$ such that $\left(\delta_{s_{1}} \epsilon^{-1} c_{1}\right)\left(\delta_{s_{2}} \epsilon^{-1} c_{2}\right)$ $\cdots\left(\delta_{s_{r}} \epsilon^{-1} c_{r}\right) x=0$ for all $c_{1}, c_{2}, \ldots, c_{r}$ in $\mathbf{g}$ and $s_{1}, s_{2}, \ldots, s_{r} \in \mathcal{O}$.

In view of 4.14, this means that $H_{r} x_{r+1} \in G_{1} W, H_{r} x_{r+2} \in G_{2} W, \ldots$

Thus, $x_{r+1} \in W_{r+1, r}, x_{r+2} \in W_{r+2, r}, \ldots$, and we have a sequence of elements in $\bar{W}_{r+1, r}, \bar{W}_{r+2, r}, \ldots$. This sequence defines an element $y \in \dot{W}_{r} \subset \dot{W}$. It is easy to check that $x \mapsto y$ is a well-defined map $\widehat{W}(-\infty) \rightarrow \dot{W}$.

A map in the opposite direction is constructed as follows.

If $t \geq \max (r, N) \geq 1$, we have a map

$$
\bar{W}_{t, r} \rightarrow W / G_{N} W
$$

induced by the inclusion $W_{t, r} \subset W$. 
The composition of the canonical map $\dot{W}_{r} \rightarrow \bar{W}_{t, r}$ with the map (a) is a map $\dot{W}_{r} \rightarrow W / G_{N} W$ which is clearly independent of the choice of $t$. These maps are compatible with the imbeddings $\dot{W}_{r} \subset \dot{W}_{r+1}$ and, hence, give rise to a C-linear map ${\underset{\lim }{\longrightarrow}}_{r} \dot{W}_{r} \rightarrow W / G_{N} W$ or equivalently $\dot{W} \rightarrow W / G_{N} W$.

The maps $\dot{W} \rightarrow W / G_{N} W$ (for various $N$ ) are compatible with the maps $W / G_{N+1} W \rightarrow W / G_{N} W$ and, hence, define a C-linear map $\dot{W} \rightarrow \widehat{W}$ whose image may be checked to be in $\widehat{W}(-\infty)$. Thus we have obtained a C-linear map $\dot{W} \rightarrow \widehat{W}(-\infty)$. One can verify that this is the inverse of the map $\widehat{W}(-\infty) \rightarrow \dot{W}$ constructed earlier.

\section{THIRD DEFINITION OF TENSOR PRODUCT}

6.1. We preserve the setup of 4.2 and the notation of $\S 4$. We shall give another definition of the tensor product. It involves something like a double dual. We will show in $\S 7$ that it coincides with the first definition of tensor product under certain finiteness conditions.

6.2. We consider the $\mathbf{C}$-vector space $Z=\operatorname{Hom}_{\mathbf{C}}(W, \mathbf{C})$ with the $\Gamma$-module structure inherited from $W$. For any $N \geq 1$, we denote by $Z^{N}$ the C-subspace of $Z$ consisting of all elements $\lambda \in Z$ such that $\xi \lambda=0$ for all $\xi \in G_{N}$. In other words, $Z^{N}$ is the annihilator of $G_{N} W \subset W$. We have $Z^{1} \subset Z^{2} \subset \cdots$ and denote by $Z^{\infty}$ the union of all $Z^{N}$.

Note that $Z^{\infty}$ is a $\Gamma$-submodule of $Z$. (Indeed, let $f \in R, c \in \mathbf{g}$, and let $\lambda \in Z^{N}$. We can find $t \geq 0$ so that $f \in R_{-t}$. By $4.9(\mathrm{c})$, we have $(f c) \lambda \in Z^{N+t}$. Thus $(f c): Z \rightarrow Z$ maps $Z^{\infty}$ into itself.)

6.3. We define a $\widehat{\mathbf{g}}^{\odot}$-module structure on $Z^{\infty}$ as follows. Let $\omega=\left(\omega_{s}\right)_{s \in \mathcal{S}} \in$ $\mathbf{C}((\epsilon))^{\ominus}$ and let $c \in \mathbf{g}$ so that

$$
\omega c \in \widehat{\mathbf{g}}^{\ominus} .
$$

Let $\lambda \in Z^{\infty}$. We can find $N \geq 1$ such that $\lambda \in Z^{N}$. We choose a function $g \in R$ such that ${ }^{s} g-\omega_{s} \in \epsilon^{N} \mathbf{C}[[\epsilon]]$ for all $s \in \mathcal{O}$. (See 4.9(a).)

We define $\langle\omega c\rangle \lambda=(g c) \lambda \in Z^{\infty}$. (Here $(g c) \lambda$ is given by the $\Gamma$-module structure of $Z^{\infty}$.) This is independent of the choice of $N$ and $g$, by 4.9(b). It is easy to verify that the endomorphisms $\langle\omega c\rangle$ define a $\widehat{\mathbf{g}}^{\ominus}$-module structure on $Z^{\infty}$ with 1 acting as multiplication by $\kappa-h$.

The restriction of the $\widehat{\mathbf{g}}^{\infty}$-module $Z^{\infty}$ to a $\widetilde{\mathbf{g}}^{\circ}$-module is denoted $T^{\prime}(W)$.

Lemma 6.4. (a) The $\widetilde{\mathbf{g}}^{\ominus}$-module $T^{\prime}(W)$ is smooth.

(b) The $\widehat{\mathbf{g}}^{\odot}$-module $Z^{\infty}$ is the $\widehat{\mathbf{g}}^{\odot}$-module attached to the smooth $\widetilde{\mathbf{g}}^{\circ}$-module $T^{\prime}(W)$ as in 1.11 .

Let $\lambda \in Z^{N}$ with $N \geq 2$. Let $c_{1}, \ldots, c_{N}$ be elements of $\mathbf{g}$, and let $s_{1}, s_{2}$, $\ldots, s_{N}$ be a sequence in $\varnothing$. Let $\omega(i) c_{i}=\delta_{s_{i}} \epsilon c_{i} \in \widehat{\mathbf{g}}^{\bigcirc}$. We can find $g \in R$ such that ${ }^{s} g-\epsilon \in \epsilon^{N} \mathbf{C}[[\epsilon]]$ for all $s \in \mathcal{O}$. 
By definition, we have $\left\langle\omega(N) c_{N}\right\rangle \lambda=\left(g c_{N}\right) \lambda$. Moreover, $\left(g c_{N}\right) \in G_{1}$ and hence $G_{N-1}\left(g c_{N}\right) \lambda \subset G_{N} \lambda=0$. Thus, $\left(g c_{N}\right) \lambda \in Z^{N-1}$. Applying the previous argument to $\left(g c_{N}\right) \lambda$ instead of $\lambda$, we obtain

$$
\left\langle\omega(N-1) c_{N-1}\right\rangle\left\langle\omega(N) c_{N}\right\rangle \lambda=\left(g c_{N-1}\right)\left(g c_{N}\right) \lambda .
$$

Continuing in this way we see that

$$
\left\langle\omega(1) c_{1}\right\rangle\left\langle\omega(2) c_{2}\right\rangle \cdots\left\langle\omega(N) c_{N}\right\rangle \lambda=\left(g c_{1}\right) \cdots\left(g c_{N}\right) \lambda
$$

The last expression is zero since $\lambda \in Z^{N}$. It follows that

$$
\left\langle\omega(1) c_{1}\right\rangle\left\langle\omega(2) c_{2}\right\rangle \cdots\left\langle\omega(N) c_{N}\right\rangle \lambda=0 .
$$

This shows that $\lambda \in T^{\prime}(W)(N)$ and (a) is proved.

If $\lambda \in Z^{N}$ and $\omega c$ as in 6.3(a) satisfies $\omega_{s} \in \epsilon^{N} \mathbf{C}[[\epsilon]]$ for all $s \in S$, then, using 4.9(b), we see that $\langle\omega c\rangle \lambda=0$. This shows that (b) holds.

The next result follows immediately from the definitions.

Lemma 6.5. We have $T^{\prime}(W)(1)=Z^{1}$.

One can also easily show that $T^{\prime}(W)(N)=Z^{N}$ for all $N \geq 1$.

6.6. The relationship between the $\widetilde{\mathbf{g}}^{\bigcirc}$-modules $T(W), T^{\prime}(W)$ can be given by two natural maps:

(a) a homomorphism of $\widetilde{\mathbf{g}}^{\bigcirc}$-modules $T(W)^{\sharp} \rightarrow D\left(T^{\prime}(W)\right)$,

(b) a homomorphism of $\widetilde{\mathbf{g}}^{\odot}$-modules $T^{\prime}(W) \rightarrow D\left(T(W)^{\sharp}\right)$.

These will be constructed in the following two subsections.

6.7. By definition, we have $Z^{N}={ }^{d}\left(W / G_{N} W\right)$. Taking again duals, we obtain ${ }^{d}\left(Z^{N}\right)={ }^{d}\left({ }^{d}\left(W / G_{N} W\right)\right)$. Hence the obvious linear map

$$
W / G_{N} W \rightarrow^{d}\left({ }^{d}\left(W / G_{N} W\right)\right)
$$

may be identified with a linear map

$$
W / G_{N} W \rightarrow^{d}\left(Z^{N}\right)
$$

This passes to projective limits and gives a linear map

$$
\underset{N}{\lim _{N}} W / G_{N} W \rightarrow \underset{N}{\lim _{N}^{d}}\left(Z^{N}\right)
$$

but

$$
\underset{N}{\lim } W / G_{N} W=\widehat{W} \text { and } \underbrace{\lim }_{N}{ }^{d}\left(Z^{N}\right)={ }^{d}\left(Z^{\infty}\right) ;
$$

hence, we have obtained a linear map

$$
\widehat{W} \rightarrow{ }^{d} T^{\prime}(W) .
$$

A routine verification using the definitions shows that this is compatible with the $\widetilde{\mathbf{g}}^{\mathcal{O}}$-module structures where ${ }^{d} T^{\prime}(W)$ is given the $\widetilde{\mathbf{g}}^{\bigcirc}$-module structure inherited from that of $T^{\prime}(W)$. 
It is clear that (e) restricts to a homomorphism of $\widetilde{\mathbf{g}}^{\odot}$-modules

$$
T(W)=\widehat{W}(-\infty) \rightarrow\left({ }^{d} T^{\prime}(W)\right)(-\infty) .
$$

Applying $\sharp$, we get the map 6.6(a).

6.8. Remark. If it were known that $\operatorname{dim}_{\mathbf{C}} W / G_{N} W<\infty$ for every $N$, so that 6.7(a) is an isomorphism for every $N$, then the previous argument shows that each of the maps 6.7(b), (c), (e) and 6.6(a) would be an isomorphism.

6.9. Let $\lambda \in T^{\prime}(W)$. Then $\lambda$ is a $\mathbf{C}$-linear form $W \rightarrow \mathbf{C}$ which is zero on $G_{N} W$ for sufficiently large $N$. Hence, it defines a linear form $\lambda_{N}$ : $W / G_{N} W \rightarrow \mathbf{C}$ for sufficiently large $N$. The composition of $\widehat{W} \rightarrow W / G_{N} W$ with $\lambda_{N}$ is a linear form $\lambda^{\prime}: \widehat{W} \rightarrow B$ which is independent of $N$. Let $\lambda^{\prime \prime}: T(W) \rightarrow \mathrm{C}$ be the restriction of $\lambda^{\prime}$ to $T(W)$. Then $\lambda \mapsto \lambda^{\prime \prime}$ is a C-linear map $T^{\prime}(W) \rightarrow^{d}(T(W))$. One can verify that it is a homomorphism of $\widetilde{\mathbf{g}}^{\odot}$-modules. Hence, it restricts to a homomorphism of $\widetilde{\mathbf{g}}^{\odot}$-modules $T^{\prime}(W)(\infty) \rightarrow\left({ }^{d} T(W)\right)(\infty)$. Since $T^{\prime}(W)(\infty)=T^{\prime}(W)$ (see 6.4), this gives a homomorphism

$$
T^{\prime}(W) \rightarrow\left({ }^{d} T(W)\right)(\infty)
$$

This is, by definition, the map $6.6(\mathrm{~b})$.

6.10. Now let $X$ be a smooth $\widetilde{\mathbf{g}}^{\odot}$-module with central charge $\kappa-h$. We can regard $X$ as a $\widehat{\mathbf{g}}^{\odot}$-module as in 1.11 and as a $\Gamma$-module via the homomorphism $\Gamma \rightarrow \widehat{\mathbf{g}}^{\odot}$ given in $4.6(\mathrm{a})$.

We will define natural isomorphisms

$$
\begin{gathered}
\operatorname{Hom}_{\widehat{\mathbf{g}} \odot}\left(X, T^{\prime}(W)\right) \cong \operatorname{Hom}_{\Gamma}(W \otimes X, \mathbf{C}), \\
\operatorname{Hom}_{\widehat{\mathbf{g}} \odot}\left(X, D\left(T(W)^{\sharp}\right)\right) \cong \operatorname{Hom}_{\widetilde{\mathbf{g}}}(T(W) \otimes X, \mathbf{C}) .
\end{gathered}
$$

Let $p: X \rightarrow Z=\operatorname{Hom}_{\mathbf{C}}(W, \mathbf{C})$ be a $\mathbf{C}$-linear map. We associate to $p$ the C-linear map $p^{\prime}: W \otimes X \rightarrow \mathbf{C}$ given by $p^{\prime}(w \otimes v)=p(v)(w)$. It is clear that $p \mapsto p^{\prime}$ is an isomorphism

$$
\operatorname{Hom}_{\mathbf{C}}(X, Z) \cong \operatorname{Hom}_{\mathbf{C}}\left(W \otimes_{\mathbf{C}} X, \mathbf{C}\right) .
$$

The condition that $p$ above is compatible with the $\Gamma$-actions on $X$ and $Z$ is that $p\left(\left({ }^{s} f c\right) v\right)(w)=-p(v)((f c) w)$ for all $c \in \mathbf{g}$, all $s \in \odot$ and all $f \in R$; this is the same as the condition that the corresponding $p^{\prime}$ is compatible with the actions of $\Gamma$.

Hence, (c) restricts to an isomorphism

$$
\operatorname{Hom}_{\Gamma}(X, Z) \cong \operatorname{Hom}_{\Gamma}(W \otimes X, \mathbf{C}) \text {. }
$$

If $q: X \rightarrow Z^{\infty}$ is a homomorphism of $\widehat{\mathbf{g}}^{\bigcirc}$-modules, then the composition of $q$ with the inclusion $Z^{\infty} \subset Z$ is a homomorphism $q^{\prime}: X \rightarrow Z$ of $\Gamma$-modules. The correspondence $q \mapsto q^{\prime}$ is then an injective C-linear map

$$
\operatorname{Hom}_{\widehat{\mathbf{g}}} \mathcal{\infty}\left(X, Z^{\infty}\right) \rightarrow \operatorname{Hom}_{\Gamma}(X, Z) .
$$


We show that it is surjective and, hence, an isomorphism. Let $q^{\prime}: X \rightarrow Z$ be a homomorphism of $\Gamma$-modules.

Since $X$ is smooth, for any $x \in X$ there exists an $N \geq 1$ such that $x \in$ $X(N)$. Then we have also $G_{N} x=0$.

Since $q^{\prime}$ commutes with the action of $\Gamma$, it follows that $q^{\prime}(x) \in Z^{N}$. Thus, the image of $q^{\prime}$ is contained in $Z^{\infty}$. Thus, there is a unique homomorphism of $\Gamma$-modules $q: X \rightarrow Z^{\infty}$ such that $q^{\prime}$ is the composition of $q$ with the inclusion $Z^{\infty} \subset Z$. By the density of $\Gamma$ in $\widehat{\mathbf{g}}^{\infty}$ (see 4.9(a)), $q$ is automatically a homomorphism of $\widehat{\mathbf{g}}^{\widehat{O}}$-modules. This proves that (e) is an isomorphism. Composing it with (d) we get the isomorphism (a).

We now essentially repeat the previous arguments with $W, \Gamma$ replaced by $T(W), \widetilde{\mathbf{g}}^{\odot}$.

The natural homomorphism analogous to $(\mathrm{c})$ :

$$
\operatorname{Hom}_{\mathbf{C}}\left(X,{ }^{d} T(W)\right) \cong \operatorname{Hom}_{\mathbf{C}}(T(W) \otimes X, \mathbf{C})
$$

restricts to an isomorphism

$$
\operatorname{Hom}_{\widetilde{\mathbf{g}}}\left(X,{ }^{d} T(W)\right) \cong \operatorname{Hom}_{\widetilde{\mathbf{g}}}(T(W) \otimes X, \mathbf{C}) .
$$

If $r: X \rightarrow\left({ }^{d} T(W)\right)(\infty)=D\left(T(W)^{\sharp}\right)$ is a homomorphism of $\widehat{\mathbf{g}}^{\mathcal{O}}$-modules, then the composition of $q$ with the inclusion $\left({ }^{d} T(W)\right)(\infty) \subset{ }^{d} T(W)$ is a homomorphism $r^{\prime}: X \rightarrow{ }^{d} T(W)$ of $\widetilde{\mathbf{g}}^{\bigcirc}$-modules. The correspondence $r \mapsto r^{\prime}$ is then an isomorphism

$$
\operatorname{Hom}_{\widehat{\mathbf{g}}^{\circ}}\left(X, D\left(T(W)^{\sharp}\right)\right) \cong \operatorname{Hom}_{\widetilde{\mathbf{g}}^{\odot}}\left(X,{ }^{d} T(W)\right) .
$$

Composing it with (d1) we get the isomorphism (b).

6.11. The homomorphism $T^{\prime}(W) \rightarrow D\left(T(W)^{\sharp}\right)$ (see 6.6(b)) gives rise to a linear map

$$
\operatorname{Hom}_{\widehat{\mathbf{g}}^{\circ}}\left(X, D\left(T(W)^{\sharp}\right)\right) \rightarrow \operatorname{Hom}_{\widehat{\mathbf{g}}} \mathcal{O}\left(X, T^{\prime}(W)\right) .
$$

Combining this with the isomorphisms $6.10(a),(b)$, we obtain a linear map

$$
\operatorname{Hom}_{\Gamma}(W \otimes X, \mathbf{C}) \rightarrow \operatorname{Hom}_{\widetilde{\mathbf{g}}}(T(W) \otimes X, \mathbf{C})
$$

or, equivalently, a linear map of coinvariants

$$
\operatorname{Hom}_{\mathbf{C}}\left((W \otimes X)_{\Gamma}, \mathbf{C}\right) \rightarrow \operatorname{Hom}_{\mathbf{C}}\left((T(W) \otimes X) \widetilde{\mathbf{g}}^{\odot}, \mathbf{C}\right) .
$$

The last map can easily be identified with the transpose of the map 4.18(a).

6.12. Remark. If the linear map 6.6(b) were known to be an isomorphism, then, by construction, the maps $6.11(\mathrm{a}),(\mathrm{b}),(\mathrm{c})$ in the previous subsection are also isomorphisms.

\section{FINITENESS FOR TENSOR PRODUCTS}

7.1. We preserve the setup of 4.2 and the notation of $\S \S 4$ and 6 .

We shall assume that for any $s \in \boldsymbol{\Lambda}$ the $\widetilde{\mathbf{g}}$-module $V_{s}$ has central charge 
$\kappa-s$ and that we can find $N_{s} \geq 1$ such that the subspace $V_{s}\left(N_{s}\right)$ is finite dimensional and generates $V_{s}$ as a $\tilde{\mathbf{g}}$-module.

[Note that, under our hypothesis that $\kappa \notin \mathbf{Q}_{\geq 0}$, the assumption above is equivalent to the assumption that the $V_{s}$ belong to $\mathscr{O}_{\kappa}$ (see 2.22); however, the hypothesis on $\kappa$ is not used in the proofs of 7.4, 7.5.]

The main result of this section is that, when applied to objects of $\mathscr{O}_{\kappa}$, the constructions of the tensor product given in $\S \S 4$ and 6 lead to the same object and that, moreover, this object is in $\mathscr{O}_{\kappa}$. (See Theorem 7.9.)

7.2. For $s \in \boldsymbol{A}$, with $s \in\left[s_{0}\right] \quad\left(s_{0} \in \mathcal{Q}\right)$, let $g_{s} \in R$ be the function defined by

(a) $g_{s}(p)=\frac{1}{1 / \gamma_{s_{0}}^{-1}(p)-1 / \gamma_{s_{0}}^{-1}\left(\gamma_{s}(0)\right)}$ for $p \in C_{s_{0}}, \quad$ and $g_{s}=0$ on $C-C_{s_{0}}$.

We have

$$
\begin{aligned}
& { }^{s} g_{s} \in \epsilon+\epsilon^{2} \mathbf{C}[[\epsilon]], \\
& s^{s^{\prime}} g_{s} \in \mathbf{C}[[\epsilon]] \quad \text { if } s^{\prime} \neq s, s^{\prime} \in \mathbf{A}, \\
& s^{\prime} g_{s}=0 \text { if } s^{\prime} \notin\left[s_{0}\right], \\
& { }^{s} g_{s}=r \epsilon^{-1}+r^{\prime} \quad \text { for some } r \in \mathbf{C}^{*}, r^{\prime} \in \mathbf{C} .
\end{aligned}
$$

7.3. We choose, for any $s_{0} \in \mathcal{O}$, a function $g\left(s_{0}\right) \in R$ such that ${ }^{s_{0}} g\left(s_{0}\right) \in$ $\epsilon+\epsilon^{2} \mathbf{C}[[\epsilon]]$ and $g\left(s_{0}\right)=0$ on $C-C_{s_{0}}$. (For example, we could take $g\left(s_{0}\right)=g_{s}$ for some $s \in\left[s_{0}\right]-\left\{s_{0}\right\}$; see 7.2.)

For any integer $N \geq 1$ we denote by $X_{N}$ the C-subspace of $U(\Gamma)$ spanned by the products $\left(g\left(s_{1}\right) c_{1}\right)\left(g\left(s_{2}\right) c_{2}\right) \cdots\left(g\left(s_{N}\right) c_{N}\right)$ with $c_{1}, c_{2}, \ldots, c_{N}$ in $\mathbf{g}$ and $s_{1}, s_{2}, \ldots, s_{N}$ in $\bigcirc$. Clearly, $X_{N} \subset G_{N}$.

We shall prove the following result:

Proposition 7.4. Let $W_{1}$ be the subspace $\bigotimes_{s \in \mathbb{w}} V_{s}\left(N_{s}\right)$ of $W=\bigotimes_{s \in \mathbb{A}} V_{s}$, where $N_{s}$ are as in 7.1. For any $M \geq 1$ we have

$$
W=W_{1}+\sum_{t=1}^{M-1} X_{t} W_{1}+G_{M} W
$$

Corollary 7.5. $W / G_{M} W$ is a finite-dimensional $\mathbf{C}$-vector space for any $M \geq 1$.

Indeed, the proposition shows that

$$
\operatorname{dim}\left(W / G_{M} W\right) \leq \sum_{t=0}^{M-1}(|\mathrm{O}| \operatorname{dim} \mathbf{g})^{t} \prod_{s \in \boldsymbol{\phi}} \operatorname{dim} V\left(N_{s}\right) .
$$

7.6. We begin the proof of the proposition by showing, by induction on $M \geq 1$, that

$$
G_{M} \subset X_{M}+\sum_{M^{\prime}: M^{\prime}>M} G_{M^{\prime}}
$$


Assume first that $M=1$. Let $c \in \mathbf{g}$ and $f \in R$ be such that for some $s_{0} \in \varnothing$ we have ${ }^{s_{0}} f-\epsilon \in \epsilon^{2} \mathbf{C}[[\epsilon]]$ and $f=0$ on $C-C_{s_{0}}$. Then, by the choice of $g\left(s_{0}\right)$, we have $f=g\left(s_{0}\right)+g^{\prime}$ where $g^{\prime} \in R_{2}$.

By 4.9(b), we have $g^{\prime} c \in G_{2}$. By definition, we have $g\left(s_{0}\right) c \in X_{1}$. Hence, $f c=g\left(s_{0}\right) c+g^{\prime} c \in X_{1}+G_{2}$. Since the elements $f c$ as above span $G_{1}$ as a vector space, we have $G_{1} \subset X_{1}+G_{2}$, as required.

Next we assume that $M \geq 2$. Using (a) with $M$ replaced by 1 and by $M-1$ and the inclusion $X_{N} \subset G_{N}$, we have

$$
\begin{aligned}
G_{1} G_{M-1} & \subset G_{1}\left(X_{M-1}+\sum_{M^{\prime} \geq M} G_{M^{\prime}}\right) \\
& \subset X_{1} X_{M-1}+G_{2} X_{M-1}+\sum_{M^{\prime} \geq M} X_{1} G_{M^{\prime}}+\sum_{M^{\prime} \geq M} G_{2} G_{M^{\prime}} \\
& \subset X_{M}+\sum_{M^{\prime}>M} G_{M^{\prime}} .
\end{aligned}
$$

(a) is proved.

7.7. Assume that $M \geq 2$ and that Proposition 7.4 is known to be true whenever $M$ is replaced by $M^{\prime}$ where $1 \leq M^{\prime}<M$. We show that it is then also true for $M$.

By our assumption, we have $W=W_{1}+G_{1} W$. It follows that $G_{M-1} W \subset$ $G_{M-1} W_{1}+G_{M-1} G_{1} W$.

Using now 7.6(a), we deduce $G_{M-1} W \subset X_{M-1} W_{1}+G_{M} W$.

Combining this with the induction hypothesis $W=W_{1}+\sum_{t=0}^{M-2} X_{t} W_{1}+$ $G_{M-1} W$ we see that

$$
W \subset W_{1}+\sum_{t=0}^{M-2} X_{t} W_{1}+X_{M-1} W_{1}+G_{M} W
$$

as required.

7.8. We are thus reduced to proving the proposition in the special case where $M=1$. The general case can immediately be reduced to the case where $C$ is connected (hence $\nabla$ has a single element). For simplicity we shall carry out the proof for $C$ connected, assuming also that consists of two elements $s_{1}, s_{2}$. The proof for general $\boldsymbol{\phi}$ is the same, but the notation is more complicated.

By our assumption, we have $V_{s}=\sum_{t \geq 0} Q_{t}^{\sharp} V\left(N_{s}\right)$ (notation of 1.7) for $s=$ $s_{1}, s_{2}$. We shall write $V_{1}, V_{2}, N_{1}, N_{2}$ instead of $V_{s_{1}}, V_{s_{2}}, N_{s_{1}}, N_{s_{2}}$. It is then enough to verify the following statement: For any $t_{1}, t_{2} \geq 0$, any $\xi_{1} \in Q_{t_{1}}^{\sharp}$, $\xi_{2} \in Q_{t_{2}}^{\sharp}$, and any $x_{1} \in V_{1}\left(N_{1}\right), x_{2} \in V_{2}\left(N_{2}\right)$, we have

$$
\xi_{1} x_{1} \otimes \xi_{2} x_{2} \in W_{1}+G_{1} W
$$

We will prove (a) by induction on $t_{1}+t_{2} \geq 0$. When $t_{1}+t_{2}=0$, (a) is trivial. Hence, we may assume that at least one of $t_{1}, t_{2}$ (say, $t_{1}$ ) is $\geq 1$. We may assume that $\xi_{1}=\left(\epsilon^{-1} c\right) \xi_{1}^{\prime}$ for some $\xi_{1}^{\prime} \in Q_{t_{1}-1}^{\sharp}$ and some $c \in \mathbf{g}$. 
By the definition of the $\Gamma$-module structure of $W$, we have

$$
\left(g_{s_{1}} c\right)\left(\xi_{1}^{\prime} x_{1} \otimes \xi_{2} x_{2}\right)=\left({ }^{s_{1}} g_{s_{1}} c\right) \xi_{1}^{\prime} x_{1} \otimes \xi_{2} x_{2}+\xi_{1}^{\prime} x_{1} \otimes\left({ }^{s_{2}} g_{s_{1}} c\right) \xi_{2} x_{2} .
$$

We have ${ }^{s_{2}} g_{s_{1}} \in \mathbf{C}[[\epsilon]]$ (see 7.2); hence, using 1.8(c),

$$
\left({ }^{s_{2}} g_{s_{1}} c\right) \xi_{2} x_{2} \in \sum_{t^{\prime}: t^{\prime} \leq t_{2}} Q_{t^{\prime}}^{\sharp} V_{2}\left(N_{2}\right) .
$$

Using the induction hypothesis for $\left(t_{1}-1, t^{\prime}\right)$ where $t^{\prime} \leq t_{2}$, we deduce that

$$
\xi_{1}^{\prime} x_{1} \otimes\left({ }^{s_{2}} g_{s_{1}} c\right) \xi_{2} x_{2} \in W_{1}+G_{1} W
$$

Recall now that ${ }^{s_{1}} g_{s_{1}}=r \epsilon^{-1}+r^{\prime}$ for some $r, r^{\prime} \in \mathbf{C}$ with $r \neq 0$ (see 7.2). Using again $1.8(\mathrm{c})$ we see that

$$
\left(r^{\prime} c\right) \xi_{1}^{\prime} x_{1} \in \sum_{t^{\prime} \leq t_{1}-1} Q_{t^{\prime}}^{\sharp} V_{1}\left(N_{1}\right),
$$

and using, as before, the induction hypothesis, we see that

$$
\left(\left({ }^{s_{1}} g_{s_{1}}-r \epsilon^{-1}\right) c\right) \xi_{1}^{\prime} x_{1} \otimes \xi_{2} x_{2} \in W_{1}+G_{1} W .
$$

This together with (c) and (b) shows that $r\left(\epsilon^{-1} c\right) \xi_{1}^{\prime} x_{1} \otimes \xi_{2} x_{2} \in W_{1}+G_{1} W$. (The left-hand side of (b) is in $G_{1} W$ since $g_{s_{1}} c \in G_{1}$, see 7.2). Since $r \neq 0$, it follows that $\xi_{1} x_{1} \otimes \xi_{2} x_{2} \in W_{1}+G_{1} W$ and the induction step is established. Proposition 7.4 is proved.

Theorem 7.9. (a) The $\widetilde{\mathbf{g}}^{\bigcirc}$-modules $T(W)^{\sharp}$ and $T^{\prime}(W)$ belong to $\mathscr{O}_{\kappa}$.

(b) The natural map $T(W)^{\sharp} \rightarrow D\left(T^{\prime}(W)\right.$ ) (see 6.6(a)) is an isomorphism of $\widetilde{\mathbf{g}}^{\odot}$-modules.

(c) The natural map $T^{\prime}(W) \rightarrow D\left(T(W)^{\sharp}\right)$ (see 6.6(b)) is an isomorphism of $\widetilde{\mathbf{g}}^{\circ}$-modules.

By 7.5, we have $\operatorname{dim} W / G_{N} W<\infty$ for any $N \geq 1$. By Remark 6.8, we see that (b) holds.

Since $T^{\prime}(W)$ is a smooth $\widetilde{\mathbf{g}}^{\circ}$-module (see $6.4(\mathrm{a})$ ) with central charge $\kappa-h$ and since $T^{\prime}(W)(1)=Z^{1}={ }^{d}\left(W / G_{1} W\right)$ is finite dimensional, we see from Theorem 3.2 that $T^{\prime}(W)$ belongs to $\mathscr{O}_{\kappa}$. Since $D$ takes $\mathscr{O}_{\kappa}$ into itself (see 2.25), we deduce that $D\left(T^{\prime}(W)\right.$ ) belongs to $\mathscr{O}_{\kappa}$; using (b), it follows that (a) holds. Now (c) follows from (a), (b), since $D$ is involutory on $\mathscr{O}_{\kappa}$ (see 2.26).

Proposition 7.10. Let $X$ be a smooth $\widetilde{\mathbf{g}}^{\circ}$-module on which 1 acts as multiplication by $\kappa-h$. We regard $X$ as $a \widehat{\mathbf{g}}^{\mathcal{O}}$-module as in 1.11 and as a $\Gamma$-module, via the homomorphism $\Gamma \rightarrow \widehat{\mathbf{g}}^{\circ}$ given in 4.6(a). If the $\mathbf{C}$-vector spaces of coinvariants $(T(W) \otimes X)_{\tilde{\mathbf{g}}}$ and $(W \otimes X)_{\Gamma}$ are finite dimensional, then the natural 
linear map

$$
(T(W) \otimes X)_{\tilde{\mathbf{g}}} \rightarrow(W \otimes X)_{\Gamma}
$$

(see 4.18(a)) is an isomorphism.

From (e) and Remark 6.12, we deduce that the natural map $\operatorname{Hom}_{\mathbf{C}}\left((W \otimes X)_{\Gamma}, \mathbf{C}\right) \rightarrow \operatorname{Hom}_{\mathbf{C}}\left((T(W) \otimes X)_{\tilde{\mathbf{g}}}, \mathbf{C}\right)$ (the transpose of the map 4.18(a)) is an isomorphism. The proposition follows.

7.11. We now assume that $C$ has precisely two connected components or, equivalently, that $\odot$ consists of two elements $s^{\prime}, s^{\prime \prime}$. Let $S^{\prime}=\left[s^{\prime}\right]$ and $S^{\prime \prime}=\left[s^{\prime \prime}\right]$. Then $S^{\prime}, S^{\prime \prime}$ form a partition of $S$. Let ${\mathcal{O}^{\prime}}^{\prime}=\left\{s^{\prime}\right\},{\mathcal{O}^{\prime \prime}}^{\prime \prime}=\left\{s^{\prime \prime}\right\}$, $\boldsymbol{A}^{\prime}=S^{\prime}-\left\{s^{\prime}\right\}$, and $\boldsymbol{\Phi}^{\prime \prime}=S^{\prime \prime}-\left\{s^{\prime \prime}\right\}$. Let $C^{\prime}, C^{\prime \prime}$ be the connected components of $C$ corresponding to $s^{\prime}, s^{\prime \prime}$ respectively.

Note that $C^{\prime}, C^{\prime \prime}$ inherit systems of charts $\left(\gamma_{s}\right)_{s \in S^{\prime}},\left(\gamma_{s}\right)_{s \in S^{\prime \prime}}$ from $C$. Thus $\left(S^{\prime}, \mathcal{O}^{\prime}, \mathbf{Q}^{\prime}, C^{\prime}, \ldots\right)$ and $\left(S^{\prime \prime},{\mathcal{O}^{\prime \prime}}^{\prime \prime}, \mathbf{\phi}^{\prime \prime}, C^{\prime \prime}, \ldots\right)$ are data of the same kind as $(S, \odot, \uparrow, C, \ldots)$ in 4.2. We can define the Lie algebras $\Gamma^{\prime}, \Gamma^{\prime \prime}$ in terms of these data in the same way as $\Gamma$ was defined in terms of the datum in 4.2. Similarly, the subspaces $G_{N}^{\prime} \subset U\left(\Gamma^{\prime}\right)$ and $G_{N}^{\prime \prime} \subset U\left(\Gamma^{\prime \prime}\right)$ are defined like the subspaces $G_{N} \subset U(\Gamma)$.

As in 4.8, $W^{\prime}=\bigotimes_{s \in \boldsymbol{\star}^{\prime}} V_{s}$ (resp. $W^{\prime \prime}=\bigotimes_{s \in \boldsymbol{A}^{\prime \prime}} V_{s}$ ) has a natural filtration $G_{N}^{\prime} W^{\prime}$ (resp. $G_{N}^{\prime \prime} W^{\prime \prime}$ ) and corresponding completion $\widehat{W}^{\prime}$ (resp. $\widehat{W}^{\prime \prime}$ ). As in 4.11, we get from this a well-defined $\widetilde{\mathbf{g}}$-module $T\left(W^{\prime}\right)$ (resp. a $\widetilde{\mathbf{g}}$-module $\left.T\left(W^{\prime \prime}\right)\right)$. These modules have the same central charge; hence, $T\left(W^{\prime}\right) \otimes T\left(W^{\prime \prime}\right)$ is naturally a $\widetilde{\mathbf{g}}^{\odot}$-module (as in 1.13).

We have the following result.

Proposition 7.12. We have a natural isomorphism of $\widetilde{\mathbf{g}}^{\odot}$-modules

$$
T\left(W^{\prime}\right) \otimes T\left(W^{\prime \prime}\right) \cong T(W) .
$$

Let $\left(x_{1}, x_{2}, \ldots\right)$ and $\left(y_{1}, y_{2}, \ldots\right)$ be two sequences of elements in $W^{\prime}$, $W^{\prime \prime}$ respectively such that $x_{r+1}-x_{r} \in G_{r}^{\prime} W^{\prime}$ and $y_{r+1}-y_{r} \in G_{r}^{\prime \prime} W^{\prime \prime}$ for all $r \geq 1$. Define a sequence $\left(z_{1}, z_{2}, \ldots\right)$ of elements in $W=W^{\prime} \otimes W^{\prime \prime}$ by $z_{1}=x_{1} \otimes y_{1}, z_{2}=x_{2} \otimes y_{2}, \ldots$. We have

$$
z_{r+1}-z_{r}=\left(x_{r+1}-x_{r}\right) \otimes y_{r+1}+x_{r} \otimes\left(y_{r+1}-y_{r}\right) \in G_{r} W .
$$

Moreover, if $x_{r} \in G_{r}^{\prime} W^{\prime}$ for all $r \geq 1$ or if $y_{r} \in G_{r}^{\prime \prime} W^{\prime \prime}$ for all $r \geq 1$, then $z_{r} \in G_{r} W$ for all $r \geq 1$.

Thus $\left(\left(x_{1}, x_{2}, \ldots\right),\left(y_{1}, y_{2}, \ldots\right)\right) \mapsto\left(z_{1}, z_{2}, \ldots\right)$ defines a C-linear map

$$
\widehat{W}^{\prime} \otimes \widehat{W}^{\prime \prime} \rightarrow \widehat{W} .
$$

We want to construct a map in the opposite direction. For any object $X$ in $\mathscr{O}_{\kappa}$ (relative to $\widetilde{\mathbf{g}}^{\mathcal{O}}$ ) we have 


$$
\begin{aligned}
\operatorname{Hom}_{\mathbf{C}}\left((W \otimes X)_{\Gamma}, \mathbf{C}\right) & =\operatorname{Hom}_{\mathbf{C}}\left((T(W) \otimes X)_{\left.\widetilde{\mathbf{g}}^{\odot}, \mathbf{C}\right)}\right. \\
& =\operatorname{Hom}_{\widetilde{\mathbf{g}}^{\odot}}\left(T(W),{ }^{d} X\right) \\
& =\operatorname{Hom}_{\widetilde{\mathbf{g}}^{\odot}}\left(T(W)^{\sharp},{ }^{d} X^{\sharp}\right) \\
& =\operatorname{Hom}_{\widetilde{\mathbf{g}}^{\mathcal{O}}}\left(T(W)^{\sharp},{ }^{d} X^{\sharp}(\infty)\right) \\
& =\operatorname{Hom}_{\widetilde{\mathbf{g}}^{\circ}}\left(T(W), D(X)^{\sharp}\right) .
\end{aligned}
$$

The first equality is by 6.12 , which is applicable in view of $7.9(\mathrm{c})$; the second and third are obvious; the fourth is because $T(W)^{\sharp}$ is smooth; the fifth is obvious.

We apply this with $X=D\left(T\left(W^{\prime}\right)^{\sharp}\right) \otimes D\left(T\left(W^{\prime \prime}\right)^{\sharp}\right)$ (see 2.28(a)). Using $X=$ $D\left(T\left(W^{\prime}\right)^{\sharp} \otimes T\left(W^{\prime \prime}\right)^{\sharp}\right)$ (see 2.28(b)) and $D(X)=T\left(W^{\prime}\right)^{\sharp} \otimes T\left(W^{\prime \prime}\right)^{\sharp}$ (see 2.26), we obtain

$$
\begin{gathered}
\operatorname{Hom}_{\mathbf{C}}\left(\left(W \otimes\left(D\left(T\left(W^{\prime}\right)^{\sharp}\right) \otimes D\left(T\left(W^{\prime \prime}\right)^{\sharp}\right)\right)_{\Gamma}, \mathbf{C}\right)\right. \\
=\operatorname{Hom}_{\widetilde{\mathbf{g}}^{\ominus}}\left(T(W), T\left(W^{\prime}\right) \otimes T\left(W^{\prime \prime}\right)\right) .
\end{gathered}
$$

Similarly, we have

$$
\operatorname{Hom}_{\mathbf{C}}\left(\left(W^{\prime} \otimes D\left(T\left(W^{\prime}\right)^{\sharp}\right)_{\Gamma^{\prime}}, \mathbf{C}\right)=\operatorname{Hom}_{\widetilde{\mathbf{g}}}\left(T\left(W^{\prime}\right), T\left(W^{\prime}\right)\right)\right.
$$

and

$$
\operatorname{Hom}_{\mathbf{C}}\left(\left(W^{\prime \prime} \otimes D\left(T\left(W^{\prime \prime}\right)^{\sharp}\right)_{\Gamma^{\prime \prime}}, \mathbf{C}\right)=\operatorname{Hom}_{\widetilde{\mathbf{g}}}\left(T\left(W^{\prime \prime}\right), T\left(W^{\prime \prime}\right)\right) .\right.
$$

To the identity map of $T\left(W^{\prime}\right)$ there corresponds under (c) a $\Gamma^{\prime}$-linear map $b^{\prime}: W^{\prime} \otimes D\left(T\left(W^{\prime}\right)^{\sharp} \rightarrow \mathrm{C}\right.$; to the identity map of $T\left(W^{\prime \prime}\right)$ there corresponds under (d) a $\Gamma^{\prime \prime}$-linear map $b^{\prime \prime}: W^{\prime \prime} \otimes D\left(T\left(W^{\prime \prime}\right)^{\sharp} \rightarrow \mathbf{C}\right.$.

The tensor product $b^{\prime} \otimes b^{\prime \prime}$ is a $\Gamma$-linear map

$$
W \otimes\left(D\left(T\left(W^{\prime}\right)^{\sharp}\right) \otimes D\left(T\left(W^{\prime \prime}\right)^{\sharp}\right) \rightarrow \mathbf{C},\right.
$$

and this corresponds under (b) to a $\widetilde{\mathbf{g}}^{\circ}$-linear map $T(W) \rightarrow T\left(W^{\prime}\right) \otimes T\left(W^{\prime \prime}\right)$. One can verify that this is the inverse of the map (a). The proposition follows.

\section{Change OF RINGS IN TENSOR PRODUCTS}

8.1. In this section we assume that we are given a commutative $\mathrm{C}$-algebra $B$ with 1 .

Most of the definitions and results of $\S \S 4,6,7$ extend with only minor changes to the case where $C$ is replaced by $B$. We will review those definitions and results in this more general context, and we will point out the places where special care should be taken.

8.2. We place ourselves in the setup of 4.2. For any $s \in S$ we tensor the homomorphism $R \rightarrow \mathbf{C}((\epsilon))$ (given by $f \mapsto{ }^{s} f$, see $4.5(\mathrm{a})$ ) by $B$, and we obtain a $B$-algebra homomorphism $B \otimes R \rightarrow B \otimes \mathbf{C}((\epsilon))$. Composing this last homomorphism with the obvious $B$-algebra homomorphism $B \otimes \mathbf{C}((\epsilon)) \rightarrow$ $B((\epsilon))$, we obtain a $B$-algebra homomorphism

$$
B \otimes R \rightarrow B((\epsilon)) \quad\left(f \mapsto^{s} f\right) .
$$


We consider the $B$-Lie algebra $\Gamma_{B}=B \otimes \Gamma$. We have natural $B$-Lie algebra homomorphisms

$$
\Gamma_{B} \rightarrow \widehat{\mathbf{g}}_{B}^{\odot}
$$

$$
\Gamma_{B} \rightarrow \widehat{\mathbf{g}}_{B}^{\infty}
$$

defined in terms of the maps (a), just as $4.6(a),(b)$.

8.3. We assume that we are given a collection of $\widetilde{\mathbf{g}}_{B}$-modules $\underline{V}_{s} \quad(s \in \mathbf{\phi})$ with the same central charge $\kappa-h$, indexed by the set $\$$.

We regard $\underline{W}=\bigotimes_{s \in \mathbb{A}} \underline{V}_{s}$ (tensor product over $B$ ) as a $\widehat{\mathbf{g}}_{B}^{\mathbf{\phi}}$-module, as in 1.13 and as a $\Gamma_{B}$-module via the homomorphism $8.2(\mathrm{~b})$. We have a natural homomorphism of $\mathbf{C}$-Lie algebras $\Gamma \rightarrow \Gamma_{B}\left(x \mapsto 1 \otimes_{B} x\right)$; we identify an element of $\Gamma$ with the corrresponding element of $\Gamma_{B}$. Via this homomorphism, we may regard $\underline{W}$ also as a $\Gamma$-module. Hence the $B$-submodules $G_{N} \underline{W}$ of $\underline{W}$ are well defined; they form a descending filtration of $\underline{W}$ and we may form, as in 4.8, the projective limit $\underline{\widehat{W}}=\lim _{N} \underline{W} / G_{N} \underline{W}$.

8.4. Let $\omega=\left(\omega_{s}\right)_{s \in \odot} \in B((\epsilon))^{\odot}$, and let $c \in \mathbf{g}$. Then $\omega c \in \widehat{\mathbf{g}}_{B}^{\odot}$. We choose a sequence $g_{1}, g_{2}, \ldots$ of elements of $B \otimes R$ such that ${ }^{s} g_{n}-\omega_{s} \in \epsilon^{n} B[[\epsilon]]$ for $n=1,2, \ldots$ and all $s \in \varnothing$. (Compare 4.9(a).) We also choose $q \geq 0$ such that $\omega_{s} \in \epsilon^{-q} B[[\epsilon]]$ for all $s \in \varnothing$.

We define a $B$-linear map $\langle\omega c\rangle: \underline{\widehat{W}} \rightarrow \underline{\widehat{W}}$ by setting

$$
\langle\omega c\rangle\left(x_{1}, x_{2}, \ldots\right)=\left(\left(g_{1} c\right) x_{q+1},\left(g_{2} c\right) x_{q+2}, \ldots\right)
$$

for any element of $\underline{\widehat{W}}$ represented by a sequence $\left(x_{1}, x_{2}, \ldots\right)$ of elements in $\underline{W}$ such that $x_{n+1}-x_{n} \in G_{n} \underline{W}$ for $n=1,2, \ldots$. (Here, $\left(g_{n} c\right) x_{q+n}$ is defined by the $\Gamma_{B}$-module structure of $\underline{W}$.)

As in $4.9,4.10$, we see that the $B$-linear maps $\langle\omega c\rangle: \underline{\widehat{W}} \rightarrow \underline{\widehat{W}}$ are well defined and that they give a $\widehat{\mathbf{g}}_{B}^{\odot}$-module structure on $\underline{\widehat{W}}$ in which $\mathbf{1}$ acts as multiplication by $-\kappa+h$.

We now restrict the $\widehat{\mathbf{g}}_{B}^{\odot}$-module $\underline{\underline{W}}$ to $\widetilde{\mathbf{g}}_{B}^{\odot}$ using the obvious imbedding $\widetilde{\mathbf{g}}_{B}^{\odot} \subset \widehat{\mathbf{g}}_{B}^{\odot}$ and we consider the $\widetilde{\mathbf{g}}_{B}^{\odot}$-submodule $T(\underline{W})=\underline{\widehat{W}}(-\infty)$ of the $\widetilde{\mathbf{g}}_{B}^{\odot}$ module $\underline{\underline{W}}$ (see 4.11). $\widetilde{\mathbf{g}}_{B}^{\odot}$-module $T(\underline{W})^{\sharp}$ (see 1.5) has central charge $\kappa-h$.

8.5. Now let $\underline{X}$ be a smooth $\widetilde{\mathbf{g}}_{B}^{\circ}$-module on which $\mathbf{1}$ acts as multiplication by $(\kappa-h)$. Then $\underline{X}$ extends naturally to a $\widehat{\mathbf{g}}_{B}^{\odot}$-module which then can be restricted to a $\Gamma_{B}$-module using the homomorphism of $B$-Lie algebras $\Gamma_{B} \rightarrow \widehat{\mathbf{g}}_{B}^{\odot}$ (see 8.2(b)).

The tensor product $\underline{W} \otimes_{B} \underline{X}$ of the $\Gamma_{B}$-modules $\underline{W}, \underline{X}$ is then a $\Gamma_{B}$-module in the standard way; it is clear that 1 acts as zero on this $\Gamma_{B}$-module. 
On the other hand, the tensor product $T(\underline{W}) \otimes_{B} \underline{X}$ of the $\widetilde{\mathbf{g}}_{B}^{\circ}$-modules $T(\underline{W}), \underline{X}$ is a $\widetilde{\mathbf{g}}_{B}^{O}$-module in the standard way; again it is clear that $\mathbf{1}$ acts as zero on this $\widetilde{\mathbf{g}}_{B}^{\mathrm{O}}$-module.

Just as in 4.16, there is a canonical $B$-linear map

such that

$$
\nu: T(\underline{W}) \otimes_{B} \underline{X} \rightarrow\left(\underline{W} \otimes_{B} \underline{X}\right) / G_{1}\left(\widehat{W} \otimes_{B} \underline{X}\right)
$$

where $\left(x_{1}, x_{2}, \ldots\right) \in T(\underline{W}), \quad z \in \underline{X}(N)$, and $t \geq N$

Just as in 4.18, this induces a $B$-linear map

$$
\left(T(\underline{W}) \otimes_{B} \underline{X}\right)_{\widetilde{\mathbf{g}}_{B}^{\odot}} \rightarrow\left(\underline{W} \otimes_{B} \underline{X}\right)_{\Gamma_{B}}
$$

8.6. As in 6.2, we now consider the $B$-module $\underline{Z}=\operatorname{Hom}_{B}(\underline{W}, B)$ with the $\Gamma_{B}$-module structure inherited from $\underline{W}$. For any $N \geq 1$, we denote by $\underline{Z}^{N}$ the $B$-submodule of $\underline{Z}$ consisting of all elements $\lambda \in \underline{Z}$ such that $\xi \lambda=0$ for all $\xi \in G_{N}$. Equivalently, $\underline{Z}^{N}$ is the annihilator of $G_{N} \underline{W} \subset \underline{W}$. We have $\underline{Z}^{1} \subset \underline{Z}^{2} \subset \cdots$ and denote by $\underline{Z}^{\infty}$ the union of all $\underline{Z}^{N}$.

As in $6.2, \underline{Z}^{\infty}$ is a $\Gamma_{B}$-submodule of $\underline{Z}$.

As in 6.3, there is a natural $\widehat{\mathbf{g}}_{B}^{\infty}$-module structure on $\underline{Z}^{\infty}$, defined as follows. Let $\omega=\left(\omega_{s}\right)_{s \in \odot} \in B((\epsilon))^{\odot}$ and let $c \in \mathbf{g}$ so that $\omega c \in \widehat{\mathbf{g}}_{B}^{\odot}$.

Let $\lambda \in \underline{Z}^{N}$. We choose $g \in B \otimes R$ such that ${ }^{s} g-\omega_{s} \in \epsilon^{N} B[[\epsilon]]$ for all $s \in O$.

We define $\langle\omega c\rangle \lambda=(g c) \lambda \in \underline{Z}^{\infty}$. (Here $(g c) \lambda$ is given by the $\Gamma_{B}$-module structure of $Z^{\infty}$.) This gives the required $\widehat{\mathbf{g}}_{B}^{\infty}$-module structure on $\underline{Z}^{\infty} ; \mathbf{1}$ acts as multiplication by $\kappa-h$.

The restriction of the $\widehat{\mathbf{g}}_{B}^{\infty}$-module $\underline{Z}^{\infty}$ to a $\widetilde{\mathbf{g}}_{B}^{\infty}$-module is denoted $T^{\prime}(\underline{W})$. As in 6.4 , the $\widetilde{\mathbf{g}}_{B}^{\odot}$-module $T^{\prime}(\underline{W})$ is smooth and the $\widehat{\mathbf{g}}_{B}^{\odot}$-module $\underline{Z}^{\infty}$ is the $\widehat{\mathbf{g}}_{B}^{\mathcal{O}}$-module attached to the $\widetilde{\mathbf{g}}_{B}^{\odot}$-module $T^{\prime}(\underline{W})$ as in 1.11 .

It is clear that $T^{\prime}(\underline{W})(1)=\underline{Z}^{1}$.

8.7. As in 6.6, we have natural homomorphisms of $\widetilde{\mathbf{g}}_{B}^{\odot}$-modules

$$
\begin{gathered}
T(\underline{W})^{\sharp} \rightarrow D\left(T^{\prime}(\underline{W})\right), \\
T^{\prime}(\underline{W}) \rightarrow D\left(T(\underline{W})^{\sharp}\right) .
\end{gathered}
$$

Moreover, as in 6.8, the map (a) is an isomorphism, provided that for any $N \geq 1$ the $B$-module $\underline{W} / G_{N} \underline{W}$ is reflexive; that is, the natural map $\underline{W} / G_{N} \underline{W} \rightarrow$ ${ }^{d}\left({ }^{d}\left(\underline{W} / G_{N} \underline{W}\right)\right)$ is an isomorphism.

\subsection{Let $\underline{X}$ be as in 8.5.}

As in 6.12 , we see that, if the $B$-linear map $8.7(\mathrm{~b})$ were known to be an isomorphism, then the $B$-linear map

$$
\operatorname{Hom}_{B}\left(\left(\underline{W} \otimes_{B} \underline{X}\right)_{\Gamma_{B}}, B\right) \rightarrow \operatorname{Hom}_{B}\left(\left(T(\underline{W}) \otimes_{B} \underline{X}\right)_{\widetilde{\mathbf{g}}_{B}^{\odot}}, B\right)
$$

given by the transpose of $8.5(\mathrm{a})$ would be an isomorphism. 
8.9. In the remainder of this section we assume that $V_{s}$ are objects of $\mathscr{O}_{\kappa}$ indexed by $s \in \boldsymbol{\Lambda}$ and such that, for all $s \in \boldsymbol{\Lambda}, \underline{V}_{s}=B \otimes V_{s}$ as $\widetilde{\mathbf{g}}_{B}$-modules.

By the right exactness of tensor product, we have for all $N \geq 1$

$$
\underline{W} / G_{N} \underline{W}=B \otimes\left(W / G_{N} W\right),
$$

where $W$ is as in 4.4; since $\operatorname{dim}\left(W / G_{N} W\right)<\infty$ (see 7.5), it follows that $\underline{W} / G_{N} \underline{W}$ is a finitely generated free $B$-module, and in particular it is reflexive. Hence, by 8.7 , we have a natural isomorphism of $\widetilde{\mathbf{g}}_{B}^{\odot}$-modules

$$
T(\underline{W})^{\sharp} \cong D\left(T^{\prime}(\underline{W})\right) .
$$

8.10. We show that we have a natural isomorphism of $\widetilde{\mathbf{g}}_{B}^{\odot}$-modules

$$
B \otimes T^{\prime}(W) \cong T^{\prime}(\underline{W}) .
$$

Taking duals in $8.9(\mathrm{a})$, we obtain

$$
\operatorname{Hom}_{B}\left(\underline{W} / G_{N} \underline{W}, B\right)=B \otimes \operatorname{Hom}_{\mathbf{C}}\left(W / G_{N} W, \mathbf{C}\right)
$$

(since $W / G_{N} W$ is finite dimensional) or, equivalently, $\underline{Z}^{N}=B \otimes Z^{N}$. Since inductive limits commute with base change, it follows that $\lim _{N \geq 1} \underline{Z}^{N}=B \otimes \lim _{N \geq 1} Z^{N}$ or, equivalently, $T^{\prime}(\underline{W})=B \otimes T^{\prime}(W)$, as required.

8.11. We have a natural isomorphism of $\widetilde{\mathbf{g}}_{B}^{\odot}$-modules

$$
B \otimes D\left(T^{\prime}(W)\right) \cong D\left(B \otimes T^{\prime}(W)\right) .
$$

This follows from Lemma 8.16, which is applicable since $T^{\prime}(W)$ is in $\mathscr{O}_{\kappa}$.

8.12. We have a natural isomorphism of $\widetilde{\mathbf{g}}_{B}^{\odot}$-modules

$$
T(\underline{W})^{\sharp} \cong B \otimes T(W)^{\sharp} .
$$

This is obtained as a composition

$$
T(\underline{W})^{\sharp}=D\left(T^{\prime}(\underline{W})\right)=D\left(B \otimes T^{\prime}(W)\right)=B \otimes D\left(T^{\prime}(W)\right)=B \otimes T(W)^{\sharp}
$$

where the first equality comes from $8.9(\mathrm{~b})$, the second from $8.10(\mathrm{a})$, the third from $8.11(\mathrm{a})$, and the fourth follows by tensoring with $B$ from $6.6(\mathrm{c})$.

8.13. We have a natural isomorphism of $\widetilde{\mathbf{g}}_{B}^{\circ}$-modules

$$
T^{\prime}(\underline{W}) \cong D\left(T(\underline{M})^{\sharp}\right)
$$

which coincides with the map $8.7(\mathrm{~b})$.

This is obtained as the composition

$$
T^{\prime}(\underline{W})=D\left(D\left(T^{\prime}(\underline{W})\right)\right)=D\left(T(\underline{W})^{\sharp}\right) .
$$

The first equality comes from 8.10 (a) and 2.26; the second comes from 8.9 (b). 
8.14. Let $\underline{X}$ be as in 8.5. As we have seen in 8.13 , the map 8.7 (b) is an isomorphism. This implies, by Remark 8.8, that the map 8.8(a) given by the transpose of the map 8.5(a) is an isomorphism.

Hence if $\underline{X}$ is such that both

$$
\left(\underline{W} \otimes_{B} \underline{X}\right)_{\Gamma_{B}} \text { and }\left(T(\underline{W}) \otimes_{B} \underline{X}\right)_{\widetilde{\mathbf{g}}_{B}^{\odot}}
$$

are reflexive $B$-modules, then the $B$-linear map

$$
\left(T(\underline{W}) \otimes_{B} \underline{X}\right)_{\widetilde{\mathbf{g}}_{B}} \rightarrow\left(\underline{W} \otimes_{B} \underline{X}\right)_{\Gamma_{B}}
$$

(see 8.5(a)) is an isomorphism.

8.15. We conclude this section with a result relating duality with change of rings. (This result has been used in 8.11.)

Let $V$ be an object of $\mathscr{O}_{\kappa}$. Then $B \otimes V$ is naturally a $\widetilde{\mathbf{g}}_{B}$-module. Let

$$
B \otimes D(V) \rightarrow D(B \otimes V)
$$

be the $B$-linear map which associates to $b \otimes \mu$ (with $\mu \in D(V)$ and $b \in B$ ) the $B$-linear form $B \otimes V \rightarrow B$ given by $b^{\prime} \otimes x \mapsto \mu(x) b b^{\prime}$. Clearly, (a) is a homomorphism of $\widetilde{\mathbf{g}}_{B}$-modules.

Lemma 8.16. The map 8.15(a) is an isomorphism of $\widetilde{\mathbf{g}}_{B}$-modules.

We have $B \otimes V=\bigoplus_{\lambda \in \mathbf{C}}\left(B \otimes \lambda_{\lambda} V\right)$; this follows from the corresponding statement (2.20) over C. Consider the following two families of submodules of $B \otimes V:$ the first family consists of the submodules $Q_{N}^{\sharp}(B \otimes V)$ for $N=1,2, \ldots$; the second family consists of the submodules $\bigoplus_{\Re \lambda \geq t} B \otimes_{\lambda} V$ for $t$ running through the real numbers. Then

any submodule in the first family contains some submodule in the second family; any submodule in the second family contains some submodule in the first family.

This follows from the corresponding property over $\mathbf{C}$, asserted in 2.21 . By definition, a $B$-linear form $B \otimes V \rightarrow B$ is in $D(B \otimes V)$ precisely when it vanishes on some submodule in the first family. By (b), this is equivalent to the condition that the linear form vanishes on some submodule in the second family; by 2.20 , this is also equivalent to the condition that the linear form vanishes on all but finitely many submodules $B \otimes_{\lambda} V$.

Thus we have naturally $D(B \otimes V)=\bigoplus_{\lambda}\left({ }^{d}\left(B \otimes{ }_{\lambda} V\right)\right)$.

On the other hand, we have $B \otimes D(V)=\bigoplus_{\lambda \in \mathbf{C}}\left(B \otimes_{\lambda} D(V)\right)$ since $D(V)$ is in $\mathscr{O}_{\kappa}$. It is then enough to prove that for each $\lambda$, the natural map

$$
B \otimes{ }_{\lambda} D(V) \rightarrow{ }^{d}\left(B \otimes_{\lambda} V\right)
$$

is an isomorphism. But this map is the composition of the isomorphism $B \otimes$ ${ }_{\lambda} D(V) \cong\left({ }^{d}\left(B \otimes{ }_{\lambda} V\right)\right)$ (induced by the isomorphism ${ }_{\lambda} D(V) \rightarrow{ }^{d}\left({ }_{\lambda} V\right)$ (see 2.23) with the natural isomorphism $B \otimes{ }^{d}\left({ }_{\lambda} V\right) \cong{ }^{d}\left(B \otimes \lambda_{\lambda} V\right)$ (which comes from the fact that ${ }_{\lambda} V$ is finite dimensional over $\mathbf{C}$ ). The lemma is proved. 


\section{REFERENCES}

[BPZ] A. A. Belavin, A. N. Polyakov, and A. B. Zamolodchikov, Infinite conformal symmetries in two-dimensional quantum field theory, Nuclear Phys. B 241 (1984), 333-380.

[BFM] A. Beilinson, B. Feigin, and B. Mazur, Introduction to algebraic field theory on curves, preprint.

[D] V. G. Drinfeld, On quasitriangular quasi-Hopf algebras closely related to $G a l(\bar{Q} / Q)$, Algebra Anal. 2 (1990), 149-181.

[K] V. G. Kac, Infinite dimensional Lie algebras, Birkhäuser, Boston, MA, 1983.

[KR] V. G. Kac and A. K. Raina, Bombay lectures on highest weight representations of infinite dimensional Lie algebras, World Scientific, Singapore and Teaneck, NJ, 1988.

[KL] D. Kazhdan and G. Lusztig, Affine Lie algebras and quantum groups, Internat. Math. Res. Notes 2 (1991), 21-29.

[KZ] V. G. Knizhnik and A. B. Zamolodchikov, Current algebra and Wess-Zumino models in two dimensions, Nuclear Phys. B 247 (1984), 83-103.

[MS] G. Moore and N. Seiberg, Classical and conformal field theory, Comm. Math. Phys. 123 (1989), 177-254.

[P] P. Polo, Projective versus injective modules over graded Lie algebras and a particular parabolic category $O$ for affine Kac-Moody algebras, preprint.

[RW] A. Rocha-Caridi and N. R. Wallach, Projective modules over graded Lie algebras, Math. Z. 180 (1982), 151-177.

[So] W. Soergel, Construction of projectives and reciprocity in an abstract setting, preprint.

[TK] A. Tsuchiya and Y. Kanie, Vertex operators in conformal field theory on P1 and monodromy representations of braid groups, Advanced Stud. Pure Math., vol. 16, Academic Press, Boston, MA, 1988, pp. 297-372.

[TUY] A. Tsuchiya, K. Ueno, and Y. Yamada, Conformal field theory on universal family of stable curves with gauge symmetries, Advanced Stud. Pure Math., vol. 19, Academic Press, Boston, MA, 1989, pp. 459-565.

Department of Mathematics, Harvard University, Cambridge, Massachusetts 02138 E-mail address: kazhdan@math.harvard.edu

Department of Mathematics, Massachusetts Institute of Technology, Cambridge, MASSACHUSETTS 02139

E-mail address: gyuri@math.mit.edu 